University of Louisville

ThinkIR: The University of Louisville's Institutional Repository

Electronic Theses and Dissertations

$12-2010$

\title{
Treatment of severe aortic stenosis : experimental design, testing, and analysis of an aortic valve bypass apical cannula.
}

Joel Douglas Graham

University of Louisville

Follow this and additional works at: https://ir.library.louisville.edu/etd

\section{Recommended Citation}

Graham, Joel Douglas, "Treatment of severe aortic stenosis : experimental design, testing, and analysis of an aortic valve bypass apical cannula." (2010). Electronic Theses and Dissertations. Paper 523.

https://doi.org/10.18297/etd/523

This Master's Thesis is brought to you for free and open access by ThinkIR: The University of Louisville's Institutional Repository. It has been accepted for inclusion in Electronic Theses and Dissertations by an authorized administrator of ThinkIR: The University of Louisville's Institutional Repository. This title appears here courtesy of the author, who has retained all other copyrights. For more information, please contact thinkir@louisville.edu. 
TREATMENT OF SEVERE AORTIC STENOSIS:

EXPERIMENTAL DESIGN, TESTING, AND ANALYSIS OF AN AORTIC VALVE BYPASS APICAL CANNULA

By

Joel Douglas Graham

B.S., University of Louisville, 2010

\author{
A Thesis \\ Submitted to the Faculty of the \\ University of Louisville \\ J. B. Speed School of Engineering \\ As Partial Fulfillment of the Requirements \\ for the Professional Degree
}

MASTER OF ENGINEERING

Department of BioEngineering

December 2010 

TREATMENT OF SEVERE AORTIC STENOSIS:

EXPERIMENTAL DESIGN, TESTING, AND ANALYSIS OF AN AORTIC VALVE BYPASS APICAL CANNULA

Submitted by:

Joel D. Graham

A Thesis Approved On

(Date)

by the Following Reading and Examination Committee:

Steven C. Koenig, Thesis Director

Keith Sharp

Guruprasad Giridharan 


\section{ACKNOWLEDGMENTS}

To try and list all the people in my life that have shaped who I am as a person and given me the opportunities I have been blessed with, could not be contained to just one page. The experiences and memories I have made throughout my collegiate journey have been some of the best of my life. I thank you all from the bottom of my heart.

I want to first specifically thank my parents and family whose support and love have carried me through my endeavors, even if some of them weren't my best ideas. Special thanks to Dr. Koenig for providing the tremendous opportunity to work with biodevices. I can still remember almost 5 years ago approaching you after an introduction to bioengineering class asking to volunteer in your lab.

To Dr. Slaughter, the experiences and knowledge I have gained from working with your research is more than I could ever learn in any course. To Mike Sobieski, thanks for being the greatest mentor I have ever had and sharing so much with me. Thanks to the both of you as well as to all the nurses, doctors, and patients for sharing a passion for improving lives during my co-op experiences. To all the wonderful people at Advocate Christ Medical Center, Jewish Hospital, and the Cardiovascular Innovation Institute thank you for making every day an adventure.

Finally, thanks to my classmates, Dr. Keynton, Guru, and the entire BioEngineering Department for all the hard work in developing the program. I am fortunate to have stumbled into being in the first class of bioengineers from the University of Louisville. I am very proud of our achievements. 


\section{ABSTRACT}

Aortic Valve Bypass surgery has found renewed interest to researchers due to its application in high-risk patients with severe aortic stenosis. This therapy consists of construction of a conduit containing a bioprosthetic or mechanical valve from the apex of the left ventricle to the descending thoracic aorta. Blood is ejected from the left ventricle via both the native stenotic aortic valve and the conduit. This thesis proposes a design for one of the least developed components of this system, the cannula component that sits inside the left ventricle and allows for flow of blood from the left ventricle and into the conduit. Experimental use of mock flow loop testing and computational flow dynamics analysis (ANSYS Inc., Canonsburg, PA, U.S.A.) on prototype and currently used clinical venous cannulas were used in the development and design of the proposed apical cannula. Aortic valve bypass results in a low gradient across the native stenotic valve and a small gradient across the conduit. The results from the mock loop experiments, CFD analysis, clinical data, and findings from previous researchers have aided in design of a new apical cannula. It is hoped that this cannula will be a more favorable design over currently used apical connectors. Further in vitro and in vivo studies are needed to validate these results. 


\section{TABLE OF CONTENTS}

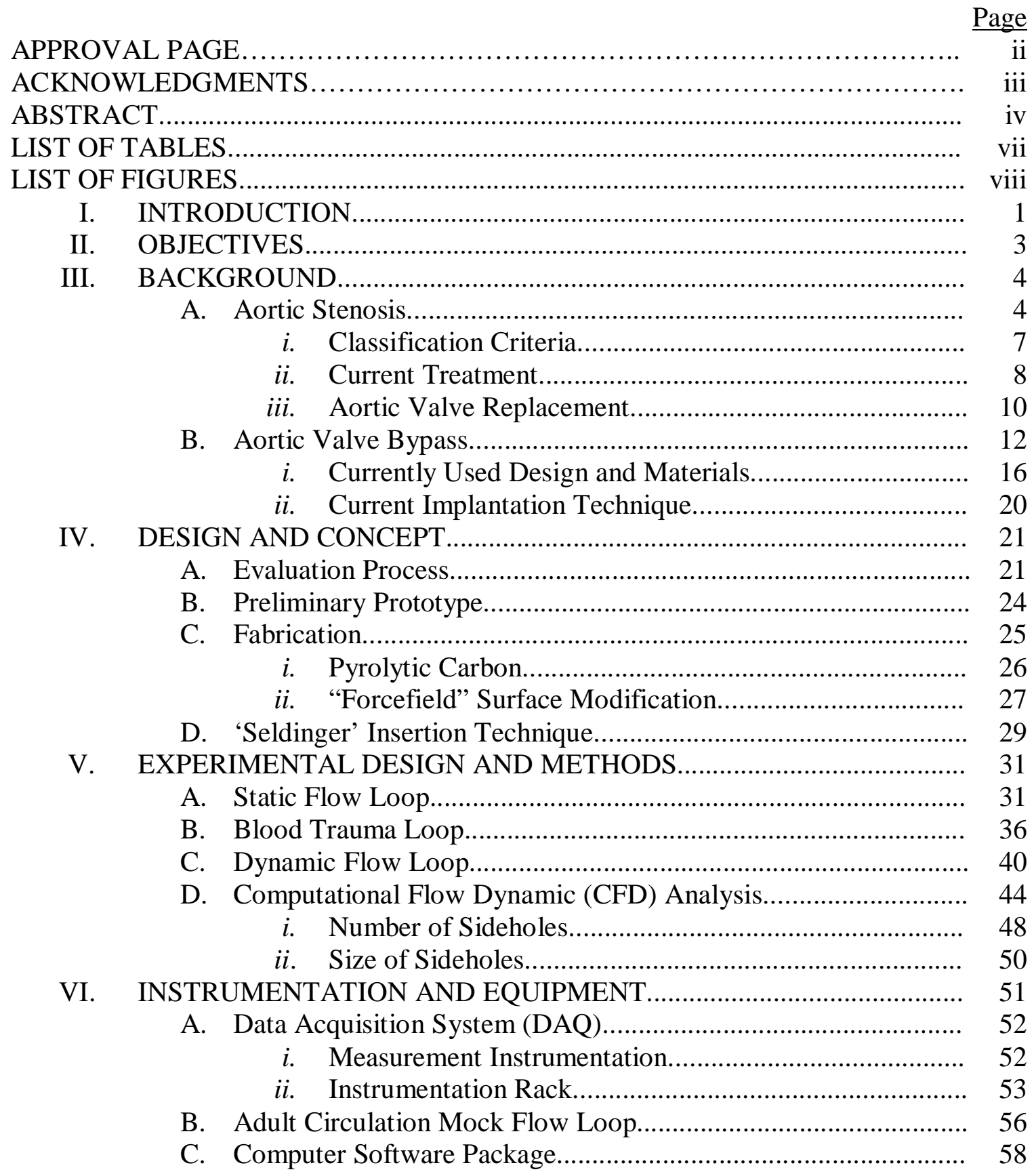


TABLE OF CONTENTS

(CONTINUED)

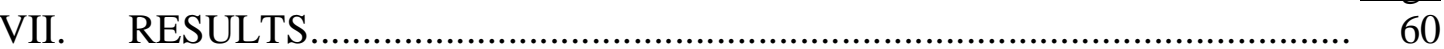

A. Static Flow Loop.................................................................. 60

B. Blood Trauma Loop................................................................. 61

C. Dynamic Flow Loop.................................................................. 62

D. Computational Flow Dynamics (CFD).................................... 65

$i . \quad$ Number of Sideholes Study........................................ 65

ii. $\quad$ Size of Sideholes Study.............................................. 69

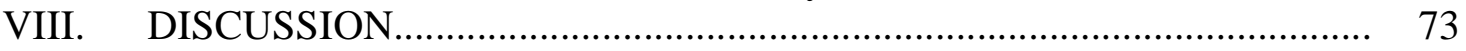

A. Static Flow Loop................................................................. 73

B. Blood Trauma Loop................................................................... 76

C. Dynamic Flow Loop.................................................................... 77

D. Computational Flow Dynamic (CFD) Analysis.......................... 78

$i . \quad$ Evaluation of Cannulas.................................................. 79

ii. Wall Shear Stress.......................................................... 81

iii. Effect of No Fillet.......................................................... 85

E. Additional Benefits of AVB Technology................................... 86

F. Type of Flow.......................................................................... 88

G. Cannula Size............................................................................ 89

H. Tip and Sideholes................................................................. 91

IX. CONCLUSIONS ........................................................................ 91

A. Static Flow Loop............................................................. 91

$i . \quad$ Limitations......................................... 92

B. Blood Trauma Loop............................................................ 92

$i . \quad$ Limitations........................................ 93

C. Dynamic Flow Loop........................................................... 94

$i . \quad$ Limitations........................................ 95

D. Computational Flow Dynamic (CFD) Analysis......................... 95

$i . \quad$ Limitations.................................................... 96

E. Summary of Experimental Results............................................. 97

X. FINAL APICAL CANNULA DESIGN.............................................. 99

A. Apical Cannula Design.......................................................... 99

B. Aortic Valve Bypass System................................................... 100

C. Closing Comments.............................................................. 102

D. Future Work....................................................................... 102

REFERENCES CITED................................................................................... 103

APPENDIX I. DYNAMIC FLOW LOOP DATA........................................ 107

APPENDIX II. SAMPLE HEMODYNAMIC WAVEFORMS............................ 108

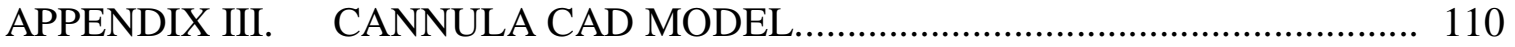

APPENDIX IV. SAMPLE CFD REPORT DATA............................................. 111 


\section{LIST OF TABLES}

Table I - Statistics for AVR, Source: STS database 2008........................................ 11

Table II - Review of Clinical Experience with Aortic Valve Bypass............................ 15

Table III - Design Criteria for the development of the apical cannula......................... 22

Table IV - Characteristics of Cannula Tested..................................................... 35

Table V - Testing conditions for the Dynamic Flow Study........................................ 42

Table VI - Cannula characteristics.................................................................... 43

Table VII - Simulation Operating Conditions........................................................... 47

Table VIII - Mesh Characteristics for Number of Sideholes Study............................ 49

Table IX - Area and Mesh Characteristics for Side of Sideholes Study........................ 51

Table X - Static loop results for Tested Cannulas..................................................... 61

Table XI - Results from Hemolysis Mock Loop Testing............................................62

Table XII - Flow rates for cannula under testing conditions...................................... 63

Table XIII - Results of Number of Sideholes Study............................................. 69

Table XIV - Results of Size of Sideholes Study...................................................... 73

Table XV - Summary of Results from Cannula Studies............................................. 97 


\section{LIST OF FIGURES}

Figure 1 - Location of Aortic valve in the heart which controls the flow of blood from the left ventricle to the Aorta (left). Transverse view of a normal aortic valve seen from the top (center). Similar view of a stenotic aortic valve (right).

Figure 2 - Prevalence of valvular disease in general population based on age. Note the steep increase in prevalence rate in those 65 years of age and older.

Figure 3 - Long term survival rates for AVR for over 13,000 patients as published by Blackstone et al.

Figure 4 - Diagram of Aortic Valve Bypass system currently used. Blood is shunted from the left ventricle into the descending aorta.............................................. 13

Figure 5 - Diagram of Aortic Valve Bypass system currently used. System consists of: A - Elbow apical connector, B - Prosthetic or mechanical valve, C - Straight woven Dacron vascular graft 17

Figure 6 - Right angle apical connector manufactured by Hancock- Extracorporeal (Hancock, Medtronic Inc., Minneapolis, MN)........................................... 18

Figure 7 - Example of valved conduit used in the AVB system produced by Medtronic, Inc.

Figure 8 - AVB system shown as an electrical circuit. Left - the left ventricle is represented as the voltage source with the aortic valve and AVB conduit being represented with a switch and resistance $\left(R_{a v}\right.$ and $\left.R_{A v B}\right)$. The basic relationships between the systems are shown in upper right table. The total resistance of $R_{a v}$ and $\mathrm{R}_{\mathrm{AVB}}$ in parallel in the aortic valve bypass model is much less than the resistance in the aortic stenosis model.

Figure 9 - Schematics, 3d models, and picture of preliminary prototype fabricated. A) Drawing of model showing dimensions and sectioned view of the cannula, B) 3D model of design (AutoCAD 2006). C) Fabricated cannula fabricated of pyrolytic carbon using a proprietary method (ATS Medical).

Figure 10 - SEM photos of platelet aggregation on "Forcefield" modified PYC and control PYC samples. The top images show clear platelet activation and spreading across the PYC while the bottom images show non-activated (round, non-attached) platelet activity. The 'Forcefield' technology is an important tool used to increase the biocompatibility of the cannula.................................... 28 


\section{LIST OF FIGURES \\ (CONTINUED)}

Figure 11 - Inflow cannula (A) designed to simplify and shorten the ventricular apical implantation procedure by using the Seldinger technique at the apex of the left ventricle (B). The conduit is then attached to the descending aorta (C). This approach eliminates the need for placing the patient on cardiopulmonary bypass, avoids ventricular coring, and achieves hemostasis through a small apical sewing ring and locking mechanism. 30

Figure 12 - Static Loop used for analysis of flows for different sized cannula under static and continuous pressure drop conditions composed of: A) Reservoir, B) Flow Loop C) DAQ system D) HeartMate II VAD system monitor. 32

Figure 13 - Static Flow loop. The white arrows show the path of the flow from the reservoir to the HeartMate II through the test section and finally out back to the reservoir. High fidelity Millar catheters were used to measure the Pre-VAD, Inlet and Outlet pressure. A Transonic flow probe was used to measure the flow rate. A calibration chamber was used to take pre and post calibration measurements.

Figure 14 - The seven cannula used separated by type and size of tip. The Cage type is distinguished by 4 main holes at the tip formed resembling a cage. The Open type features an open orifice at the tip with eight sideholes arranged on the head of the cannula. The Dome type features a smooth dome tip with eight sideholes arranged around the head of the cannula 36

Figure 15 - Flow loop used in blood hemolysis data. A) 500mL reservoir, B) Testing section, C) HeartMate II continuous flow VAD................................................. 37

Figure 16 - HemoCue Plama/Low Hb Photometer used in the hemolysis study to measure plasma free hemoglobin. 38

Figure 17 - LEFT: Bovine blood flowing through a mock flow loop consisting of A) $500 \mathrm{~mL}$ Reservior, B) Standard 23cm CPB tubing, C) HeartMate II Controller, D) HeartMate II continuous flow VAD, E) Transonic Flow Probe and Pressure Catheter inside injection port. RIGHT: Preliminary Prototype inserted inside the tubing simulating the insertion technique used for implantation.

Figure 18 - The meshing program for ANSYS CFD software analysis. A medium density meshing profile was chosen to create the meshes in the analysis 45

Figure 19 - Defining the boundary regions and initial conditions for the cannula in the Pre-solver component software. 46 


\section{LIST OF FIGURES \\ (CONTINUED)}

Figure 20 - The CAD models for the number of sideholes CFD study. Both the cannula design (left) and the model used for meshing and CFD analysis (right) are shown for the four different models

Figure 21 - The CAD models for the size of sideholes CFD study. Both the cannula design (left) and the model used for meshing and CFD analysis (right) are shown for the four different models. 50

Figure 22 - DAQ system used to collect and store data from the mock flow loop studies used to characterize the cannula....

Figure 23 - The DAQ system allows for real-time monitoring and collection of data from waveforms produced from pressure and flow transducers

Figure 24 - Adult circulation mock flow loop developed by Pantalos et al. Physiologically equivalent pressures, volumes, flows are used to reproduce normal, heart failure, and partial cardiac recovery physiologic states of the heart.

Figure 25 - Diagram of the adult circulation mock flow loop. Due to its versatility and robustness, the mock loop is able to test the AVB system providing a powerful in vitro testing environment.

Figure 26 - Flow profiles for all seven cannula tested in the static flow loop and shows resistance to flow decreases with increasing diameter size. Legend Key: C cage, $\mathrm{O}$ - open, $\mathrm{D}$ - dome. Outer French (Fr) diameter sizes are given.

Figure 27 - Difference in valve gradient (LVP - AoP) from the adult circulation mock flow loop between baseline and with the AVB system. Improvement can be seen as the cannula diameter increases. The largest improvement can be seen in the $36 \mathrm{Fr}$ cannula for the severe aortic stenosis condition.

Figure 28 - Relative conduit flow as a percentage of total cardiac output. \% Conduit flow provides a measure of the effectiveness of the cannula in the AVB system. The percentage increased as cannula size was increased...

Figure 29 - Flow rates for $12 \mathrm{~mm}$ diameter (36Fr) cannula with different numbers of sideholes. 66 


\section{LIST OF FIGURES \\ (CONTINUED)}

Figure 30 - Velocity profiles for the number of sideholes study. Four (Top Left), Eight

(Top Right), Twelve (Bottom Left), and No Sideholes (Bottom

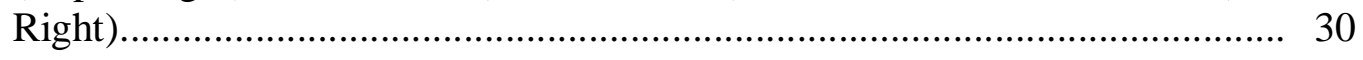

Figure 31 - Shear wall stresses for the number of sideholes study. Four (Top Left), Eight (Top Right), Twelve (Bottom Left), and No Sideholes (Bottom Right). The highest wall shear rates are seen on the bottom edges of the most distal sideholes................................................................................................... 31

Figure 32 - Flow rates for cannula in the size of sidehole study. The cannula with no fillet has the highest flow rates.

Figure 33 - Velocity profiles for the size of sideholes study. Small (Top Left), Medium (Top Right), Large (Bottom Left), No Fillet (Bottom Right) (............................................................................................... 71

Figure 34 - Shear wall stresses for the size of sidehole study. Small (Top Left), Medium (Top Right), Large (Bottom Left), No Fillet (Bottom Right).

Figure 35 - 'Open' tip design that includes an open orifice with 8 sideholes displayed the best flow characteristics from the three designs studied in static loop testing.. 74

Figure 36 - Pressure Drop vs. Flow Rate data obtained from the cannula manufacturer to the left with data obtained from static loop testing on the right. The top figure shows the 'open' tip Sarns Malleable Venous Return Catheters while the bottom shows the 'cage' tip Sarns Venous Return Catheters. The data obtained from the manufacturer closely matched data obtained and reinforces observations made in the experiment. 75

Figure 37 - Wall shear stress for all cannulas studies. Color above blue represents a shear stress level that exceeds $0.125 \mathrm{mmHg}(166$ dynes/cm2). The addition and size of sideholes increases the areas of high shear stress 81

Figure 38 - Wall shear stress for all cannulas studies. Any color below red represents a shear stress level lower than $0.02 \mathrm{mmHg}(27$ dynes/cm2). The addition and size of sideholes increases the areas of low shear stress near the tip and proximal sideholes. 83

Figure 39 - Streamlines for 12 holes cannula. 


\section{LIST OF FIGURES \\ (CONTINUED)}

Figure 40 - Velocity vector fields on cross section of Medium and No Fillet cannulas.

The No Fillet Cannula had slightly higher flow and similar shear stress properties to the Medium cannula................................................................... 85

Figure 41 - Results from Belaras et al. Through computational modeling a brain protective advantage is presented for AVB over AVR. They also conclude that cannula as small as $10 \mathrm{~mm}$ can relieve left ventricular outflow tract obstruction in critical aortic valve stenosis.

Figure 42 - Data from Vliek et al. showing upward trend in valve gradient as the cannula size decreases (Left) while there is no effect on \% flow due to cannula size (Right).

Figure 43 - Cross sectional view of the HeartMate II. Blood in the experiment were in contact with the small clearances inside the pump and may affect the results of the hemolysis study.

Figure 44 - Apical cannula design developed from mock loop studies created with CAD software (SolidWorks SP3.0, Dassault Systèmes). The selected criterion includes a $12 \mathrm{~mm}(36 \mathrm{Fr})$ outer diameter, an open tip design, and is fabricated from pyrolytic carbon. All units are in millimeters. Upper Left - Sectioned Side view, Lower Left - Top view, Lower Right - Right Sided view, Upper Right $3 \mathrm{D}$ rendered drawings.

Figure 45 - Proposed design for the AVB system. The apical cannula adapts into a right bend adaptor and into a size $17 \mathrm{~mm}$ Dacron graph. The valve intended for use is a mechanical valve also sized to $17 \mathrm{~mm}$. This model was designed through use of CAD software (SolidWorks SP3.0, Dassault Systèmes) and used in CFD analysis. 


\section{INTRODUCTION}

Treatment of aortic stenosis through surgical replacement has been one of the most successful advances in cardiovascular medicine and has increased the quality of life for patients worldwide (1). However, use of this technique in certain patient populations, specifically the elderly, has been associated with increased mortality rates (2). A growing alternative therapy for these populations is implantation of Aortic Valve Bypass (AVB) (3). In simplest terms, this alternative creates a second path for blood ejected from the left heart by creating a one-way conduit between the apex of the left ventricle and the descending aorta. The overall resistance of the blood being pumped to the rest of the body is thereby decreased by the creation of two paths to follow.

The AVB system consists of three main components: a bioprosthetic or mechanical valve, an apical cannula inserted into the left ventricular apex, and bypass conduit material connecting these components. Blood is ejected from the left ventricle via both the native stenotic aortic valve and the AVB conduit. The advantages of AVB lie in the decreased surgical risks as compared to conventional aortic valve replacement (AVR) in high-risk patient population pools. AVR requires cardiopulmonary bypass, ascending aortic crossclamping, aortotomy, debridement of the diseased valve, and cardioplegic cardiac arrest which are all avoided in the AVB surgical approach.

The focus of this report is on the design and testing of a critical component in the AVB system, the apical cannula inserted into the apex of the left ventricle. There has been much research devoted in the past into the development of the valve and conduit design $(2,4,5)$. Design and testing of artificial heart valves for surgical replacement have been refined over the course of over 4 decades while research on conduit materials have 
increased with the advent of Ventricle Assist Devices (VAD). Though, currently there is little research on the optimization of the apical cannula at the insertion point of the conduit.

Important factors in creation of the cannula include the tip design, inlet diameter size, number of sideholes, and flow profile. The novel aspects of the proposed design are in the use of Pyrolitic Carbon (PYC) for fabrication and the modified Seldinger surgical implantation technique used $(6,7)$. PYC has been used for decades in prosthetic valves and shows significant biocompatibility in the body. The modified Seldinger technique provides a simple and quick insertion procedure designed to decrease operating time and improve patient outcomes.

A series of experiments was completed to shape the design and characteristics of the prototypes. This includes use of mock flow loop testing and Computational Flow Dynamics (ANSYS Inc., Canonsburg, PA, U.S.A.). Mock loop testing was performed at the Cardiovascular Innovation Institute in Louisville, KY. The cannula was produced with the use of CAD modeling (SolidWorks SP3.0, Dassault Systèmes; Vélizy, France) and employed the use of a proprietary manufacturing process for fabrication (ATS Medical; Minneapolis, MN). This report presents a design of an apical cannula for use in aortic valve bypass. Further experimentation and animal studies will be needed in future development of this promising technology. 


\section{OBJECTIVES}

The main objective of this work is the design of a new apical cannula to be used in the AVB system. This was accomplished by testing of a series of clinically available cardiopulmonary bypass venous cannulas, a prototype cannula, and CFD analysis of computer generated cannula models. The end result of this testing is a new proposed apical cannula presented in Section X.

Section III provides a background into aortic valve bypass and details the benefits of AVB over AVR in high risk patient populations. Section IV details the design process followed in the development of the cannula, as well as the novel aspects, properties, and techniques associated with the cannula. This section also presents the preliminary prototype, a cannula produced for testing.

Sections V, VI, and VII describe the methods, instrumentation, and the results, respectively, of experiments performed on the cannulas. These testing methods included three flow loop experiments and CFD analysis. Section VIII discusses the results of the experiments and provides insight into the properties of the cannula. Section IX wraps up the work with conclusions and ideas for future work. The final section, based from the conclusions of the experiments, presents a final design for an apical cannula proposed Section X. 


\section{BACKGROUND}

The aortic valve is a semilunar, tricuspid valve positioned between the aorta and left ventricle at the end of the left ventricular outflow tract. The normal working of this valve is critical to maintaining efficient cardiac function. During ventricular systole, pressure rises in the left ventricle. When the pressure in the left ventricle rises above the pressure in the aorta, the aortic valve opens, allowing blood to exit the left ventricle into the aorta. When ventricular systole ends, pressure in the left ventricle rapidly drops and the aortic pressure forces the aortic valve to close.

The opening and closing of the aortic valve is a passive mechanism responding to the pressure fluctuations of the cardiac cycle, particularly the pressure differences between the left ventricular chamber and the aorta. Although pressure changes during the cardiac cycle may create some structural changes in the valve mechanism to facilitate opening or closing, the principal component of valvular function is the pressure difference between the left ventricle and the aorta. (8)

\section{$\underline{\text { Aortic Stenosis }}$}

Aortic Stenosis (AS) is the narrowing of the native valve controlling blood flow between the left ventricle and aorta in the heart. As the valve narrows, it impedes the flow of blood to the rest of the body and can lead to a host of physiologic complications, including congestive heart failure. When the aortic valve becomes stenotic, it causes an increase in the pressure gradient between the left ventricle (LV) and the aorta during systole. The more constricted the valve, the higher the pressure gradient between the LV and the aorta. Figure 1 shows the differences between normal and stenotic valves. 


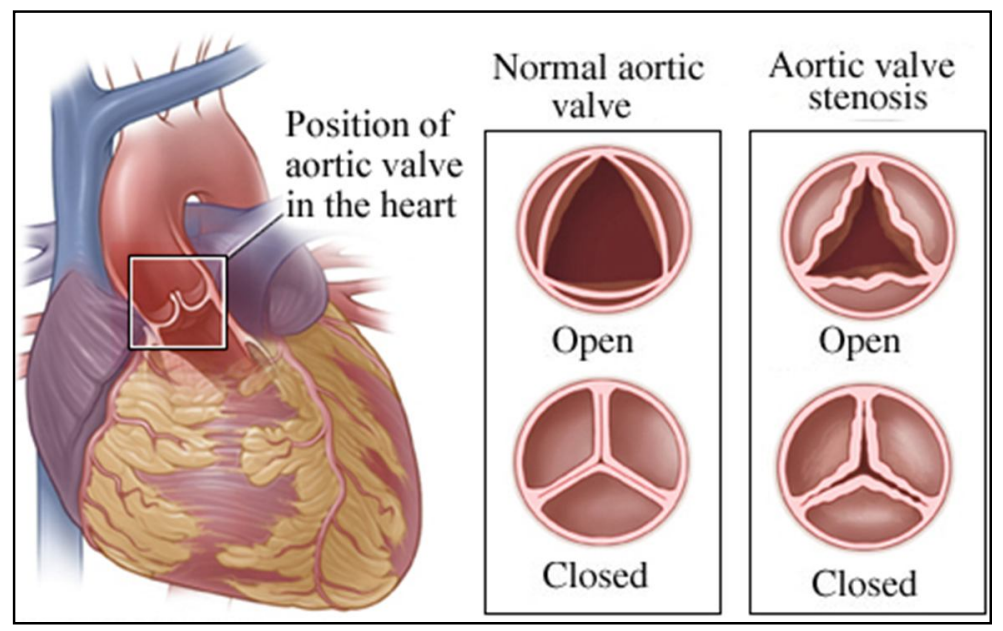

FIGURE 1 - Location of Aortic valve in the heart which controls the flow of blood from the left ventricle to the Aorta (left). Transverse view of a normal aortic valve seen from the top (center). Similar view of a stenotic aortic valve (right).

AS is the most common of valvular defects and develops mainly in the elderly population (9). It is primarily caused by three conditions: congenital defects, calcification, or scarring caused by disease.

The most common source of AS of the three main causes is degenerative calcification of the aortic valve. This is a condition where large calcium deposits form on the aortic valve leaflets. Over time the leaflets will harden and thicken, a condition called aortic sclerosis, which will develop into AS. Although previously considered to be the result of years of mechanical stress on an otherwise normal valve, it is now believed that the degenerative process leads to inflammatory changes, with lipid accumulation, upregulation of angiotensin converting enzyme (ACE) activity, and infiltration of macrophages and $\mathrm{T}$ lymphocytes ultimately leading to calcification of the aortic valve (10). 
Congenitally, many patients are born with a bicuspid or a unicommissural aortic valve, an anatomic variant which the aortic valve consists of one or two leaflets that frequently evolves into stenosis. Bicuspid aortic valves are present in approximately $2 \%$ of the general population (10). Gradual calcification of the bicuspid aortic valve results in significant stenosis most often with patients in their late 50's or 60's. This becomes more prevalent earlier in unicommissural than in bicuspid valves and earlier in men than in women. The underlying causing the development of AS in these patients is due to the abnormal architecture of the unicommissural or bicuspid aortic valve which induces turbulent flow. Turbulent flow injures the leaflets and leads to fibrosis, increased rigidity, leaflet calcification, and narrowing of the aortic valve orifice.

The last main cause in the development of AS is scarring of the heart's aortic valve which is caused by a number of factors. A large number of AS cases in the past arose from scarring induced from rheumatic fever. Rheumatic AS currently represents the least common form of AS in the adult population and usually occurs in conjunction with mitral valve stenosis (1). Rheumatic AS is characterized by diffuse fibrous leaflet thickening of the valve with fusion to a variable extent of one or two commissures.

Overall distribution of causes of AS varies significantly among different age groups. Among patients younger than 70 years of age, congenitally calcified bicuspid valves were responsible for half of surgical cases. Though, in patients over 70 years of age, degenerative calcific stenosis is the most prevalent cause of stenosis. The prevalence of AS is growing rapidly within the aging population of the United States. Nkomo et al. completed a population based study with over 12,000 randomly selected adults from the 
general population (11). They noted that moderate or severe valvular diseases are common and increase with age as seen in Figure 2.

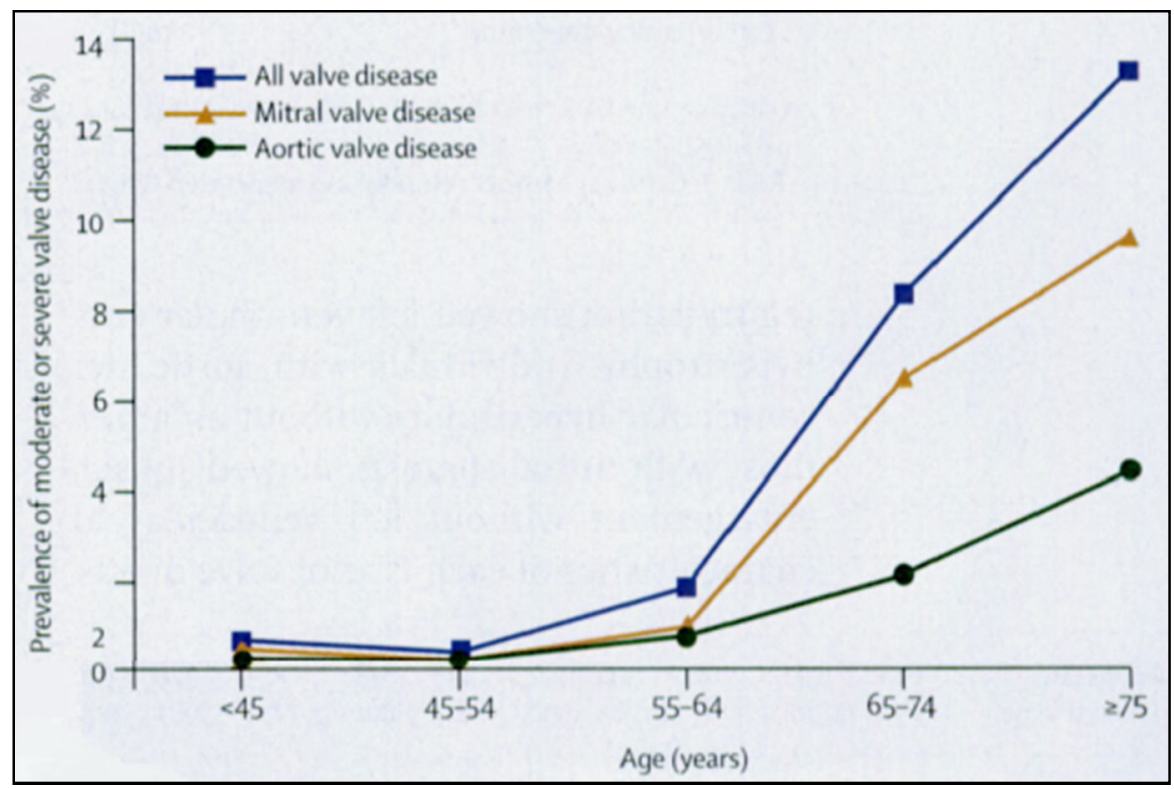

FIGURE 2 - Prevalence of valvular disease in general population based on age. Note the steep increase in prevalence rate in those 65 years of age and older.

\section{Classification Criteria for Aortic Stenosis}

Surveillance of the aortic valve for calcification and deterioration of the AS monitored by jet velocity, measurement of aortic valve area, and transvalvular pressure gradient are important clinical factors in identifying populations of AS patients at risk. The severity of AS is assessed by estimating the mean systolic gradient and aortic valve area (AVA). In 2006, the American College of Cardiology/American Heart Association Task Force on Practice Guidelines (ACC/AHA Practice Guidelines) published a report outlining the definition and care for valvular diseases (12). A classification of severity that incorporates 3 measurable factors in AS was proposed. Estimation of aortic valvular 
area and maximum aortic velocity of the blood column during systole, as well as peak and mean pressure gradients across the stenotic valve can be achieved using Doppler echocardiography and pressure catheter measurement.

In these guidelines, AS is graded as mild for AVA greater than $1.5 \mathrm{~cm}^{2}$, moderate for areas between 1.0 and $1.5 \mathrm{~cm}^{2}$, and severe for a valve area less than $1.0 \mathrm{~cm}^{2}$. The criteria for mild, moderate, and severe AS, when analyzing measured jet velocity in meters per second, are less than 3.0, 3.0 to 4.0, and greater than 4.0, respectively. The transvalvular pressure gradient is best measured with a catheter in the left ventricle and another in the proximal aorta.

The peak-to-peak gradient is measured as the difference between peak left ventricular pressure and peak aortic pressure and commonly used to quantify the valve gradient. A mean gradient across the aortic valve of less than $25 \mathrm{~mm} \mathrm{Hg}$ is classified as mild AS, severe AS is defined by a mean pressure drop greater than $40 \mathrm{~mm} \mathrm{Hg}$, and moderate AS lies between mild and severe. Most patients with AS have surgery when the AVA is less than $0.7 \mathrm{~cm}^{2}$ or there is a mean systolic gradient of more than $50 \mathrm{~mm} \mathrm{Hg}$, though patients may become symptomatic sooner and require AVR before these thresholds are reached (9).

\section{Current Treatments for AS}

To date, there is no conclusive evidence to support optimal medical management of AS and in the past have shown dismal prognosis for patients (9). Rosenhek and colleagues identified 128 consecutive patients with severe AS with an aortic jet velocity of $5.0+/-0.6 \mathrm{~m} / \mathrm{s}$ and followed them prospectively for $22+/-18$ months (13). An event 
was defined as either death (8 patients) or surgical replacement (AVR). At one year twothirds of the patients remained event free, and by four years only a third remained event free. Of those patients whose aortic jet velocity increased by more than 0.3 meters per second per year, $80 \%$ died or underwent AVR within 2 years. The severity of aortic valve calcification was an independent predictor of event-free survival. Absence of or minimal valvular calcification predicted a $75 \%$ event-free survival at 4 years. Only $20 \%$ of those with moderate to severe calcification remained event free at 4 years (13).

A larger study of 622 patients with severe, asymptomatic AS as defined by a peak jet velocity of greater than $4 \mathrm{~m} / \mathrm{s}$ were completed by Pellikka and colleagues (14). At 5year follow-up only $25 \%$ of the patients remained free of surgery or cardiac death and only one-third remained symptom free. One-third of patients developed cardiac symptoms of angina, syncope, or dyspnea before surgery within 2 years. They identified a statistical risk of sudden death of approximately $1 \%$ per year in un-operated patients (14). In large part due to data published, the ACC/AHA Practice Guidelines (12) have said, "there are no medical treatments proven to prevent or delay the disease process in the aortic valve leaflets." It seems the best treatment option for patients with severe AS is a surgical intervention, which most commonly is through aortic valve replacement (AVR).

Alternative therapies include new developments in percutaneous deployment of aortic valves in stents (percutaneous aortic valve replacement, PAVR) and a revival of a surgical treatment known as an aortic valve bypass (AVB). There are two approaches to PAVR, an antegrade and retrograde approach, which have seen some success in high risk patient groups (9). PAVR is still in its experimental stages and carries significant risks, 
though its potential as an alternate therapy is very promising. The techniques proposed in this thesis work advocate the use of aortic valve bypass for treatment in high risk patients.

\section{Aortic Valve Replacement}

The current and most routinely used treatment for severe AS is aortic valve replacement (AVR). Since its first use in 1961, the basic procedure has remained unchanged and over 180,000 procedures are now performed annually worldwide (15). The procedure for AVR surgically removes the patient's stenotic aortic valve and replaces it with either a bioprosthetic valve or a mechanical valve. AVR commonly demands the use of cardiopulmonary bypass, ascending aortic crossclamping, aortotomy, debridement of the diseased valve, and cardioplegic cardiac arrest; it therefore carries a finite risk of morbidity and mortality.

Outcomes after conventional AVR, although clearly superior to untreated AS, remain imperfect. Table I shows recent data from the 2008 Society of Thoracic Surgeons (STS) database. Operative mortality remains fairly low at $4 \%$, but increases with high risk patient groups. Although operative mortality is an important outcome for any therapeutic intervention for aortic stenosis, it is also important to consider the morbidity associated with cardiac surgery. 


\section{TABLE I}

STATISTICS FOR AVR, SOURCE: STS DATABASE 2008.

\begin{tabular}{|l|l|}
\hline AVR Statistics & Mean \\
\hline Age & 66 Years \\
\hline Reoperation & $17 \%$ \\
\hline Cross-clamp time & $80 \mathrm{~min}$ \\
\hline Perfusion time & $110 \mathrm{~min}$ \\
\hline Operative Mortality & $4 \%$ \\
\hline
\end{tabular}

In the 2001 STS report, the risk for stroke was $1.58 \%$, renal failure $3.70 \%$, and reoperation for bleeding $4.12 \%$ (2). Figure 3 shows that survival rates decrease with age (16). One report shows that for patients 80 years of age and older undergoing AVR, the immediate mortality is $10 \%$ (17). The risks of operative conditions increase the morality rates in the high-risk elderly population, though this treatment is comparatively better than no intervention.

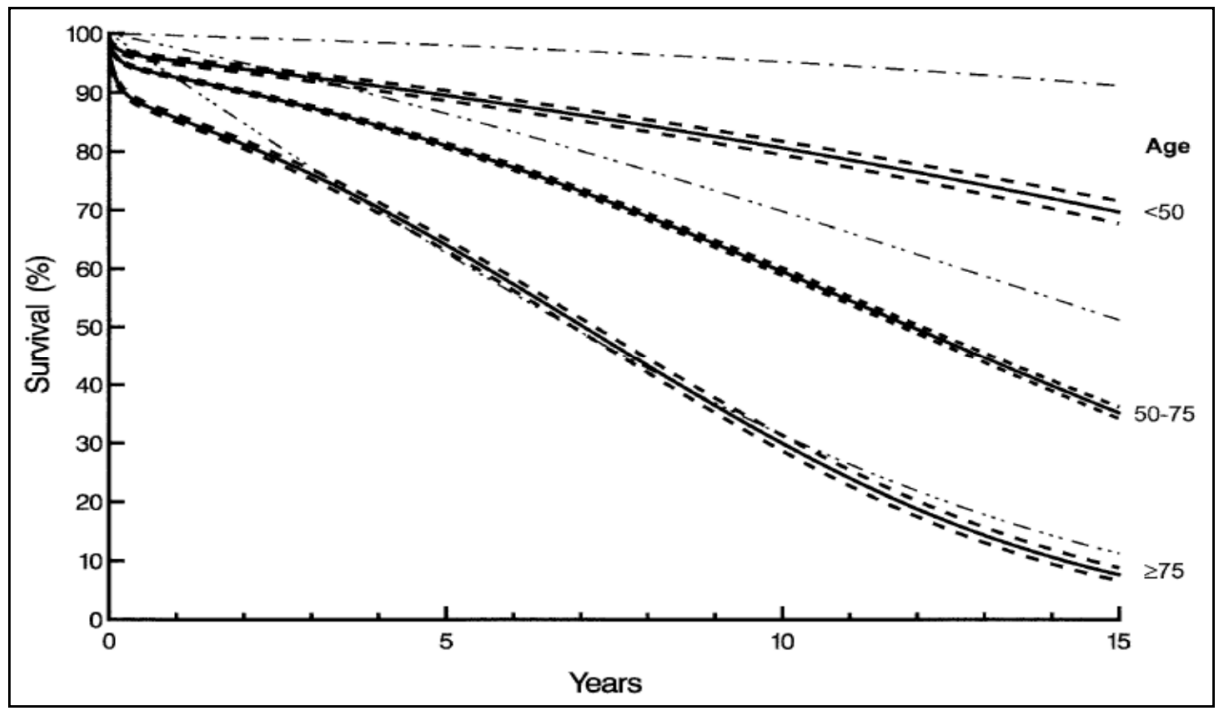

FIGURE 3 - Long term survival rates for AVR for over 13,000 patients as published by Blackstone et al. 
Despite the mortality, the number of patients older than 80 years undergoing AVR is significant. As the population ages, and without an alternative therapeutic option, surgery in these high-risk patients continues (9). For patients with severe AS who refuse surgery, the average survival is 2 years or less (18). Of the octogenarians surviving the initial postoperative period of 60 days, two-thirds survived 3 years or longer, and $40 \%$ were alive 5 or more years after surgery (17). Because symptomatic AS is an indication for surgery, alleviation of symptoms postoperatively is a factor in assessing surgical risk and benefit. In the elderly, relief of symptoms may be of even greater importance than long-term survival data. Patients and physicians alike are therefore willing to undertake AVR even in light of significant immediate mortality (9).

\section{Aortic Valve Bypass}

The surgical treatment of AS with Aortic Valve Bypass is not a new idea and has been around for 45 years. Aortic valve bypass (AVB; apicoaortic conduit) surgery relieves AS by shunting blood from the apex of the left ventricle to the descending thoracic aorta through a valved conduit (Figure 4). The idea was first conceived by Carrel in 1910 (4), performed experimentally by Sarnoff in 1955 (19) and had first clinical use by Templeton in 1962 (20). This technique has been almost exclusively used in the highrisk AS population. 


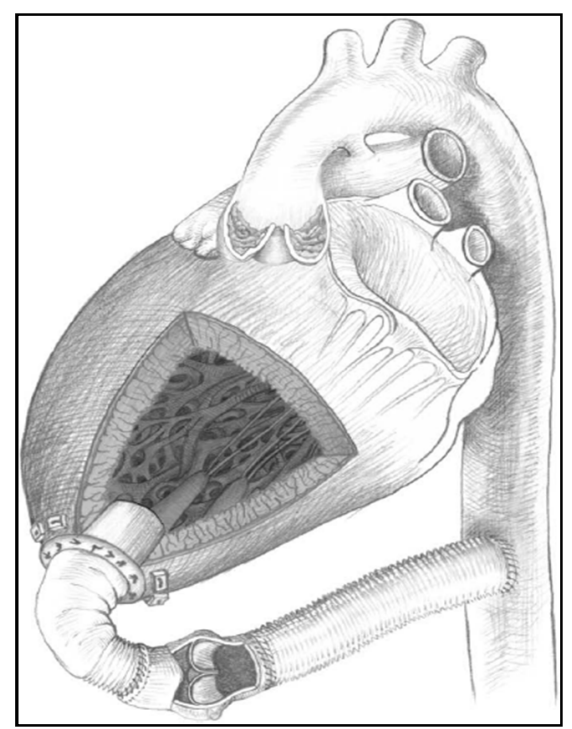

FIGURE 4 - Diagram of Aortic Valve Bypass system currently used. Blood is shunted from the left ventricle into the descending aorta.

For some high risk patients, more exclusively in the elderly population, AVB is an important alternative surgical therapy offering distinct advantages over AVR. The main advantages include avoidance of sternotomy, cardiopulmonary bypass, cardioplegic arrest, native valve debridement, conduction system injury, aortic cannulation, and aortic cross-clamping. Another advantage to clinicians, particularly over percutaneous approaches, is the ability to use a known commercially available prosthetic valve with a strong track record for durability (21). Surgeons are able to mix and match approved valves and components in the assembly of the AVB system to suit the patient and procedure used.

Gammie et al. performed a retroactive chart review of AVB and reported that this type of treatment is promising in nature and believe the basic configuration of the system to be brain protective (22). Blood flow to the brain comes from the stenotic valve while 
the rest of systemic flow is delivered from the aortic bypass. Any potential thrombi spawned from the prosthetic valve are carried downstream to the descending aorta thereby lessening the chances for stroke. Gammie, et al. provided evidence for this in a clinical review of AVB (Table II), which demonstrated complete absence of late stoke among hospital survivors of the surgery.

Clinically, the same group has recently achieved very positive results in using AVB (3). They found that the mean systolic gradient across the native aortic valve decreased from 43 to $10 \mathrm{mmHg}$ and echocardiographically determined conduit flow (expressed as a percentage of total cardiac output) was $72 \%$. Approximately one third of blood flow went through the native stenotic valve and two thirds via the conduit. They hypothesize that the distribution of blood flow after AVB surgery protects the brain from thromboembolism arising from the prosthetic valve because no blood flow to the brain is derived from the conduit. They concluded that this may contribute to a decrease in both perioperative and long-term stroke risk after AVB surgery compared with conventional AVR (3). 


\section{TABLE II}

REVIEW OF CLINICAL EXPERIENCE WITH AORTIC VALVE BYPASS.

\begin{tabular}{|c|c|c|c|c|c|}
\hline Author & Year & $\begin{array}{l}\text { No. of } \\
\text { Patients }\end{array}$ & $\begin{array}{l}\text { No. of } \\
\text { Adults }\end{array}$ & $\begin{array}{l}\text { Operative } \\
\text { Mortality }\end{array}$ & Follow-Up \\
\hline Brown $^{a}$ & 1984 & 23 & 4 & $5 / 23(22 \%)$ & $\begin{array}{l}44 \text { mos: } 13 \text { alive, class I, no reoperation } \\
4 \text { alive, class I, reoperation } 1 \text { late } \\
\text { death (infection) }\end{array}$ \\
\hline Cooley $^{\mathrm{b}}$ & 2000 & 7 & 7 & $2 / 7(29 \%)$ & ? time $5 / 7$ "well" \\
\hline Sweeney ${ }^{c}$ & 1986 & 38 & 18 & $4 / 38(10 \%)$ & $?$ \\
\hline Renzulli $^{\mathrm{d}}$ & 2000 & 4 & 4 & $1 / 4(25 \%)$ & 15 years \\
\hline Vassiliades $^{e}$ & 2000 & 3 & 3 & $0 / 3$ & 4 months \\
\hline Crestanello $^{f}$ & 2004 & 13 & 13 & $3 / 13(24 \%)$ & 2.1 years: 4 late deaths \\
\hline Freeman & 2005 & 1 & 1 & $0 / 1$ & 24 years \\
\hline Current Series & 2005 & 14 & 14 & $2 / 14(14 \%)$ & 12 months \\
\hline Totals & & 103 & 64 & $17 / 103(17 \%)$ & \\
\hline
\end{tabular}

A) Eighteen of 23 done without CPB; 18 of 23 were reoperations; 2 deaths in children with complex congenital problems; 2 deaths in patients in cardiogenic shock; 1 technical problem (80-year-old patient with friable ventricle: bleeding contributed to death); used 20 to $22 \mathrm{~mm}$ conduits for adults [22]. B) Both deaths in high-risk patients; left ventricle connector size range 14 to $20 \mathrm{~mm}$ [5]. C) 8 late deaths; 4 shunt-related (3 disruption of conduit from LV, 1 infection); 26 long-term survivors all asymptomatic, none on anticoagulation, none with thrombembolism; in subgroup of patients 65 and older, 6 of 7 were alive and well an average of 5 years postoperatively [12]. D) One death due to postoperative sepsis. Patient 2, 19 years out without symptoms, has had two babies; 3 patient had conduit thrombosis due to heavily calcified tissue valve 6 years out, underwent successful reoperation (valve replacement in conduit) and died at 13 years (suicide). Patient 4 required similar valve replacement 3 years postoperatively, now 15 years out and doing well [15]. E) All cases done off-pump [11]. F) No thromboembolic complications related to the conduit were observed among hospital survivors.

The benefits of AVB over AVR for high risk patients are numerous, though there are still potential problems with the operation. Some of these problems include pseudoaneurysm, difficulty with the aortic anastomosis in a highly calcified descending aorta, kinking of the conduit, and possible dislodgement of LV apical thrombus that was not visible in preoperative or intraoperative imaging. The surgeon may also have difficulties in the early adoption of this technique. Some of these problems include experience trouble with bleeding due to lack of control of the left ventricular apex of the 
heart and possible use of femoral cardiopulmonary bypass which adds risk of embolization. Though as Gammie et al. explain, once the initial learning curve is surmounted the surgeon should be able to perform the operation quickly, safely, and without the use of cardiopulmonary bypass (3).

\section{Currently Used Design and Materials}

There are 3 typical components used for this operation: (9) a rigid apical connector with a right-angle bend constructed of polypropylene that surrounds a woven Dacron graft and is covered with knitted Dacron and includes a sewing ring; (10) a prosthetic valve or mechanical valve (stentless porcine valves are the most commonly used valves); and (4) a straight woven Dacron vascular graft as shown in Figure 5. There are few industry-produced apparatus used for AVB surgery and as such it is often common for institutions to mix and match grafts, conduits, and connectors to suit their surgical technique. As this technique is further developed and used, further development of available materials is expected. Description and commonly used materials of these three components are given as following. 


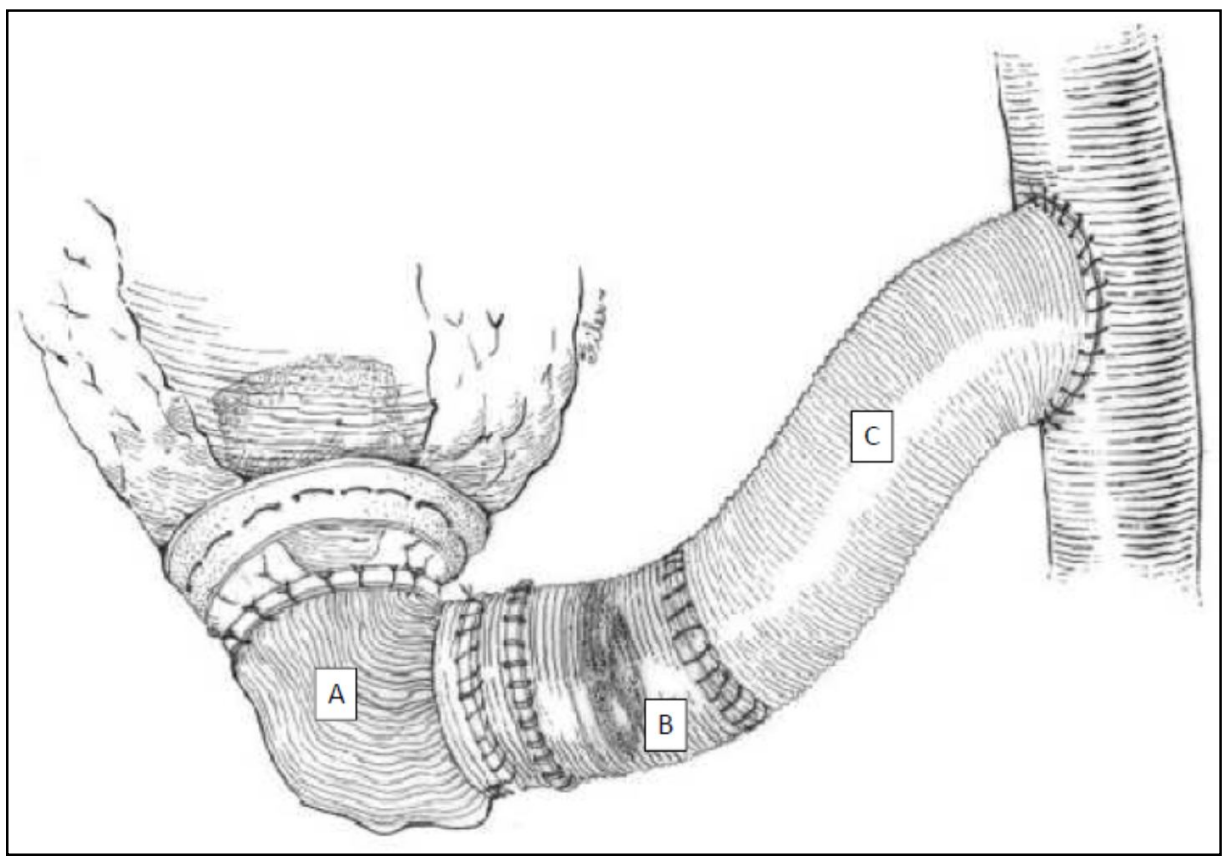

FIGURE 5 - Diagram of Aortic Valve Bypass system currently used. System consists of:

A - Elbow apical connector, B - Prosthetic or mechanical valve, C - Straight woven Dacron vascular graft. (23)

1) Apical connector - Animal laboratory work done by Brown et al. demonstrated that a semi-rigid stent was necessary to prevent progressive narrowing of the apical left ventriculotomy (23). Direct application of a prosthetic graft to the apical myocardium has been reported and resulted in obstruction at the ventriculotomy site (24). A curved left ventricular connector or stent is supplied in sizes from 12 to $22 \mathrm{~mm}$ by Medtronic, Inc., (Figure 6). 


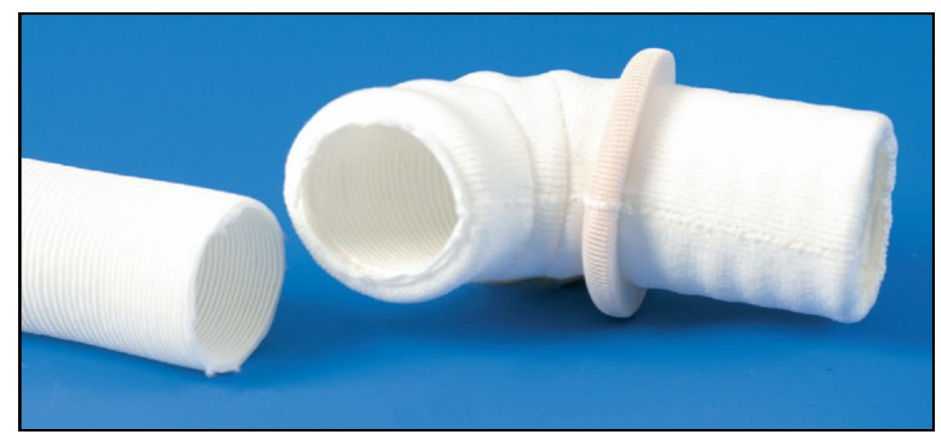

FIGURE 6 - Right angle apical connector manufactured by Hancock- Extracorporeal (Hancock, Medtronic Inc., Minneapolis, MN) (25).

The connector was developed in the early 1970s by Hancock- Extracorporeal (Hancock, Medtronic Inc., Minneapolis, MN) for Brown, et al., (20) and has been continually supplied since that time. It was originally supplied in both straight and angled configurations, though is routinely used as the right-angled connector for aortic valve bypass done through a left thoracotomy. The $90^{\circ}$ angle in the stent prevents kinking of the conduit. The apical connector is designed to project 3 to $10 \mathrm{~mm}$ into the cavity of the left ventricle. It is lined with a low-porosity woven graft and its external surface is covered with a more porous graft material to promote tissue ingrowth (23).

2) Prosthetic or mechanical valve - Either type of valve may be used, and their usage depends on the institution performing the operation. Due to widespread use and development of AVR, the design and safety of replacement aortic valves have been refined and improved for decades. The sizes range from 17 to $23 \mathrm{~mm}$ as appropriate to the patient and procedure. Currently, the valves used in most institutions for AVB are prosthetic with the most common being the stentless porcine valve. The advantages of prosthetic valves lie in the postoperative treatment of patients. Mechanical valves require 
anticoagulation drugs, such as Coumadin, which increase the risk for stroke. With use of prosthetic valves, physicians can treat patients postoperatively with just aspirin.

It is also possible to obtain valved conduits on the market that simplify the work of the surgeon as the valve and the vascular graft are preassembled. Currently, the only FDA approved commercially available valved conduit is manufactured by Medtronic, Inc. (Minneapolis, MN) seen in Figure 7.

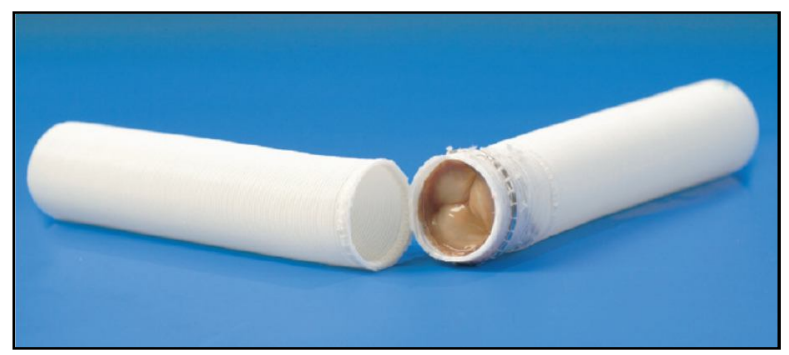

FIGURE 7 - Example of valved conduit used in the AVB system produced by Medtronic, Inc (25)

The conduit contains a stentless porcine aortic valve that is glutaraldehyde preserved and has an estimated valve area of $3 \mathrm{~cm}^{2}$. It comes in sizes of 12 to $22 \mathrm{~mm}$ in diameter, which can be used for almost any size patient. The valve in the conduit is positioned between the proximal and middle third of the conduit. This position of the valve allows room for placement of vascular clamps, when or if the conduit valve replacement is needed in late follow-up. (23)

3) Straight woven Dacron vascular graft - Awoven Dacron graft produced by Hemashield, Boston Scientific, Natick, MA is the most commonly used graft Surgeons use this graft material for many different clinical applications, including shunts, valves, and cannula (25). The length and size of the graft is custom suited to the patient. 


\section{Current Implantation Technique}

Implantation of the AVB conduit system is commonly performed through either a median sternotomy or lateral thoracotomy. The left thoracotomy is the most common approach , particularly if the patient has had prior sternotomy(s). The thoracotomy procedure has been well described by a number of physicians $(5,23,24)$. The procedure can be done without crossclamp and hypothermic circulatory arrest, and requires markedly less time on cardiopulmonary bypass. Most importantly, the risk of perioperative stroke is significantly reduced decreased (25).

A currently used 'off pump' procedure, as described by Vassiliades (5), uses an 18-gauge needle inserted through the left ventricular apex. A guide wire and a series of dilators are then applied to the ventricular apex and a 14 Fr occlusion balloon is inserted over the wire. A ventricular coring device is then threaded in-line over the catheter, and the apical core is removed. The balloon occludes the apical opening in the ventricle while the connector is slid into place. The occlusion balloon is left in place while sutures are sewed from the ventricular muscle around the opening in the apex to the external cuff of the connector. The distal end of the apical connector is then sewed to the valved conduit and de-aired (5). 


\section{DESIGN AND CONCEPT}

\section{Evaluation Process}

As previously discussed, the aortic valve bypass system is favored by many clinicians due to the established track record of their individual components (i.e. graft, valve, apical cannula). Specifically, prosthetic valves have a long history and have been successfully used clinically for decades. In contrast, the apical cannula used for AVB has not been extensively studied. The design of the current apical connectors is an adaptation from a design primarily used for complex congenital left outflow tract obstruction in children (3). As such, optimization of the design of the apical cannula could improve flow characteristics of the entire system.

There are a number of important evaluation criteria when developing the apical cannula (Table III). These important design points include the size of the conduit, biocompatibility, optimal tip design, surgical insertion technique, stagnation points, and blood hemolysis/shear stresses. Other important design criteria include the type of blood flow, amount of retrograde flow, and adequate blood flow to the aortic arch head vessels.

The upper and lower limits for the size of the cannula are governed by two factors. The upper limit is defined by the surgical insertion technique used. The lower limit is the smallest size that will allow adequate blood flow through the conduit with low aortic valve pressure gradient. To discern these design end-points, a series of in vitro experiments with a mock circulation flow loop were conducted, which were then validated in a computational flow dynamic (CFD) model. 
TABLE III

DESIGN CRITERIA FOR THE DEVELOPMENT OF THE APICAL CANNULA

\begin{tabular}{|l|c|c|c|c|c|}
\cline { 2 - 6 } \multicolumn{1}{c|}{} & \multicolumn{3}{c|}{ Mock Flow Loop } & Computational & Animal \\
Expe rimental Design Crite rion & $\begin{array}{c}\text { Dynamic } \\
\text { Flow }\end{array}$ & $\begin{array}{c}\text { Static } \\
\text { Flow }\end{array}$ & $\begin{array}{c}\text { Blood } \\
\text { Trauma } \\
\text { (CFX) }\end{array}$ & $\begin{array}{c}\text { Model } \\
\text { (bovine) }\end{array}$ \\
\hline \hline Size of the conduit & $\mathbf{X}$ & $\mathbf{X}$ & & $\mathbf{X}$ & $\mathbf{X}$ \\
\hline Biocompatibility of materials & & & $\mathbf{X}$ & & $\mathbf{X}$ \\
\hline Optimal tip design & $\mathbf{X}$ & $\mathbf{X}$ & & $\mathbf{X}$ & $\mathbf{X}$ \\
\hline Stagnation areas / eddies (thrombosis) & & & & $\mathbf{X}$ & $\mathbf{X}$ \\
\hline Blood Shear Rate / Hemolysis & & & $\mathbf{X}$ & & $\mathbf{X}$ \\
\hline Type of flow (laminar or turbulent) & & $\mathbf{X}$ & & & $\mathbf{X}$ \\
\hline Amount of retrograde flow & $\mathbf{X}$ & & & & $\mathbf{X}$ \\
\hline Flow to coronaries and head vessels & $\mathbf{X}$ & & & & $\mathbf{X}$ \\
\hline Surgical Insertion Technique & & & & & \\
\hline
\end{tabular}

It is important to have a large enough inlet diameter to allow for ample flow of blood through the conduit. It is helpful to imagine the system in terms of an electrical circuit to better understand the important factors in this analysis (Figure 8). In aortic stenosis, the resistance of the aortic valve is greatly increased and in response the flow rate decreases and AV pressure gradient increases. The AVB system creates another parallel path in the circuit. The total resistance of the aortic valve with the conduit is much less than the resistance of the stenotic aortic valve. The goal of the AVB system is to return the total resistance to normal levels so that the pressures and flow rates can return to normal physiologic conditions. 


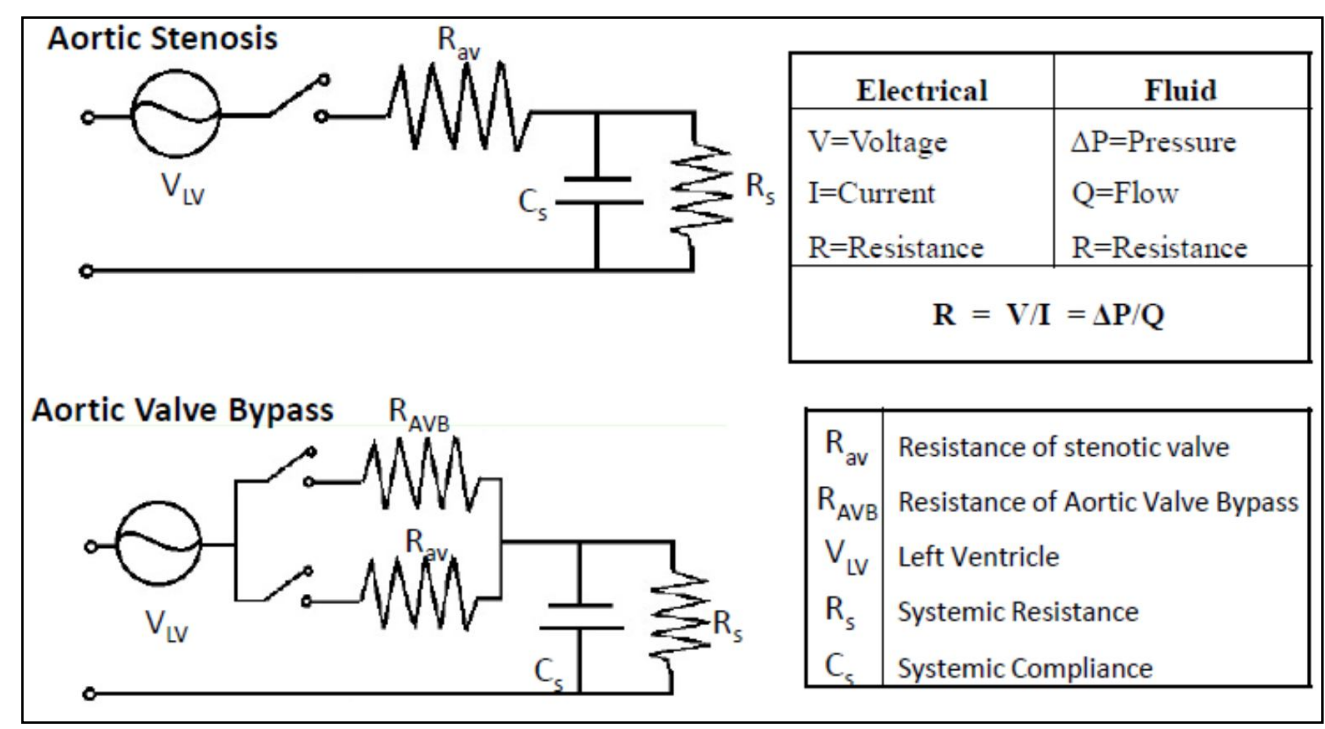

FIGURE 8 - AVB system shown as an electrical circuit. Left - the left ventricle is represented as the voltage source with the aortic valve and AVB conduit being represented with a switch and resistance $\left(\mathrm{R}_{\mathrm{av}}\right.$ and $\left.\mathrm{R}_{\mathrm{AVB}}\right)$. The basic relationships between the systems are shown in upper right table. The total resistance of $R_{a v}$ and $R_{A V B}$ in parallel in the aortic valve bypass model is much less than the resistance in the aortic stenosis model.

It is also important to find the point of highest resistance in the AVB system, as this will ultimately be the critical design feature of the device. In this case, the point of highest resistance may lie at the narrowest portion of the conduit, which in the current system is the cannula inserted into the left ventricle. The cannula is the narrowest point in the AVB system because it offers the most benefits in terms of decreased surgical risk. As such, the diameter should be as small as possible while still delivering enough blood to decrease the total resistance back to near normal values. 


\section{Preliminary Cannula Prototype}

A preliminary prototype was fabricated and tested for feasibility of concept. A mock circulation flow loop model was used to investigate the efficacy of the AVB design. Observations and data from these experiments were instrumental in the creation of a final, proposed design. The inflow cannula was constructed of Pyrolytic Carbon (PYC) and measured $7 \mathrm{~cm}$ in length with $10 \mathrm{~mm}$ outer diameter at the cannula interface that tapers to $7 \mathrm{~mm}$ outer diameter at the tip. The prototype was based in part on designs used in pediatric cardiopulmonary bypass venous cannula and was modeled with the use of CAD modeling (AutoCad 2006). Figure 9 shows the preliminary prototype CAD drawings, 3D model, and pictures of the fabricated cannula. The cannula has an open tip design with 12 side holes to allow for rapid flow of blood from the left ventricle into the descending aorta. 


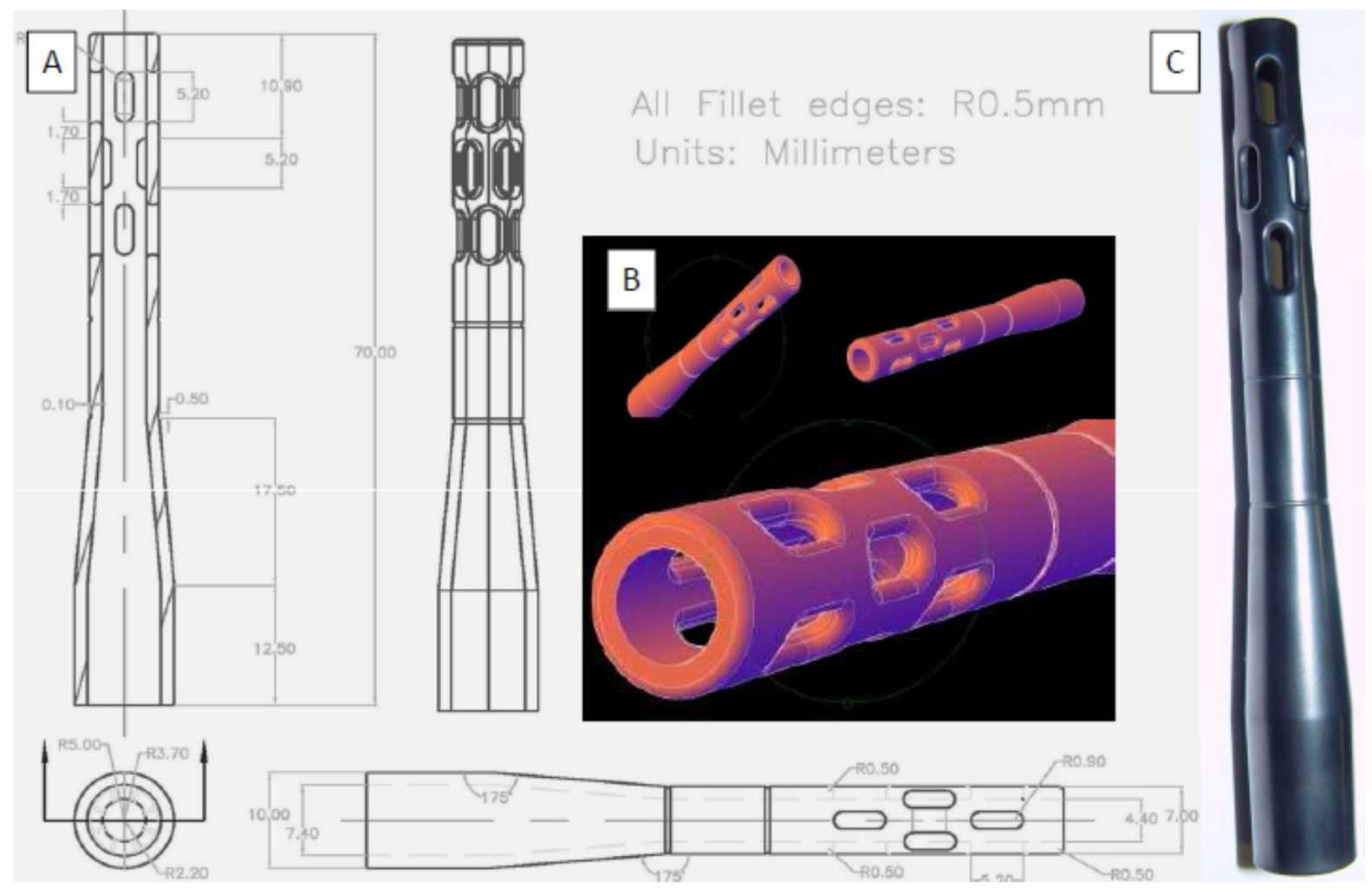

FIGURE 9 - Schematics, 3D models, and picture of preliminary prototype fabricated. A)

Drawing of model showing dimensions and sectioned view of the cannula, B) 3D model of design (AutoCAD 2006). C) Fabricated cannula fabricated of pyrolytic carbon using a proprietary method (ATS Medical).

\section{$\underline{\text { Fabrication }}$}

The fabrication utilized a proprietary rapid-prototyping manufacturing process (ATS Medical; Minneapolis, MN). The fabrication process creates a strong, brittle ceramic design with a high modulus of elasticity. It has been shown that thermal treatment of pyrolytic carbon at temperatures above $3000^{\circ} \mathrm{C}$ results in the formation of blocks having a mosaic sub-structure, which cause a reduction in the azimuthal 
disorientation of carbon produced at a temperature higher than $2000^{\circ} \mathrm{C}$ in a vacuum, corresponding to the structure of amorphized natural carbon.

\section{Pyrolytic Carbon}

Biocompatibility of implanted materials has been a concern for clinicians due to the many possible complications, including infection and rejection from the body. Pyrolytic Carbon (PYC) has been one of the most successful implantable biomaterials, having been utilized in millions of medical devices worldwide. In other medical applications, such as heart valves and orthopedic joints, PYC has high durability, strength, and resistance to wear, while also exhibiting excellent biocompatibility and thromboresistance. Today, PYC remains the most popular material available for artificial mechanical heart valves, being used to produce over 4 million devices of 25 different designs.

PYC was chosen as the fabrication material for the cannula due to these biocompatibility properties. Slaughter et al. have presented experience with a new type of proprietary surface modification known as "Forcefield ${ }^{\mathrm{TM}}$ ", (ATS Medical) (6). Details of this experiment are given below. This modification was not used in testing the cannula in this report, though this unique property was a factor in choosing PYC for prototype fabrication. 


\section{"Forcefield" surface modification}

The Forcefield surface alteration uses an electrostatic charge in conjunction with protein adsorption from blood to create a biocompatible blood interface that resists platelet adhesion. A small charge is applied to the experimental PYC over a short duration in a proprietary process (ATS Medical). A section of PYC with the applied 'forcefield' was tested clinically in a cardiopulmonary bypass circuit (CPB) circuit in six patients and showed a significant reduction in platelet adhesion and activation under scanning electromicroscopy (SEM) as seen in Figure 10.

Patients undergoing a coronary artery bypass graph (CABG) operation are exposed to both a control and 'Forcefield' modified PYC sample in an experimental section of the CPB tubing used in perfusion during cardioplegia. The results obtained from that study show the potential for an increase in biocompatibility, as well as improvement in thromboresistance for PYC. (6) 


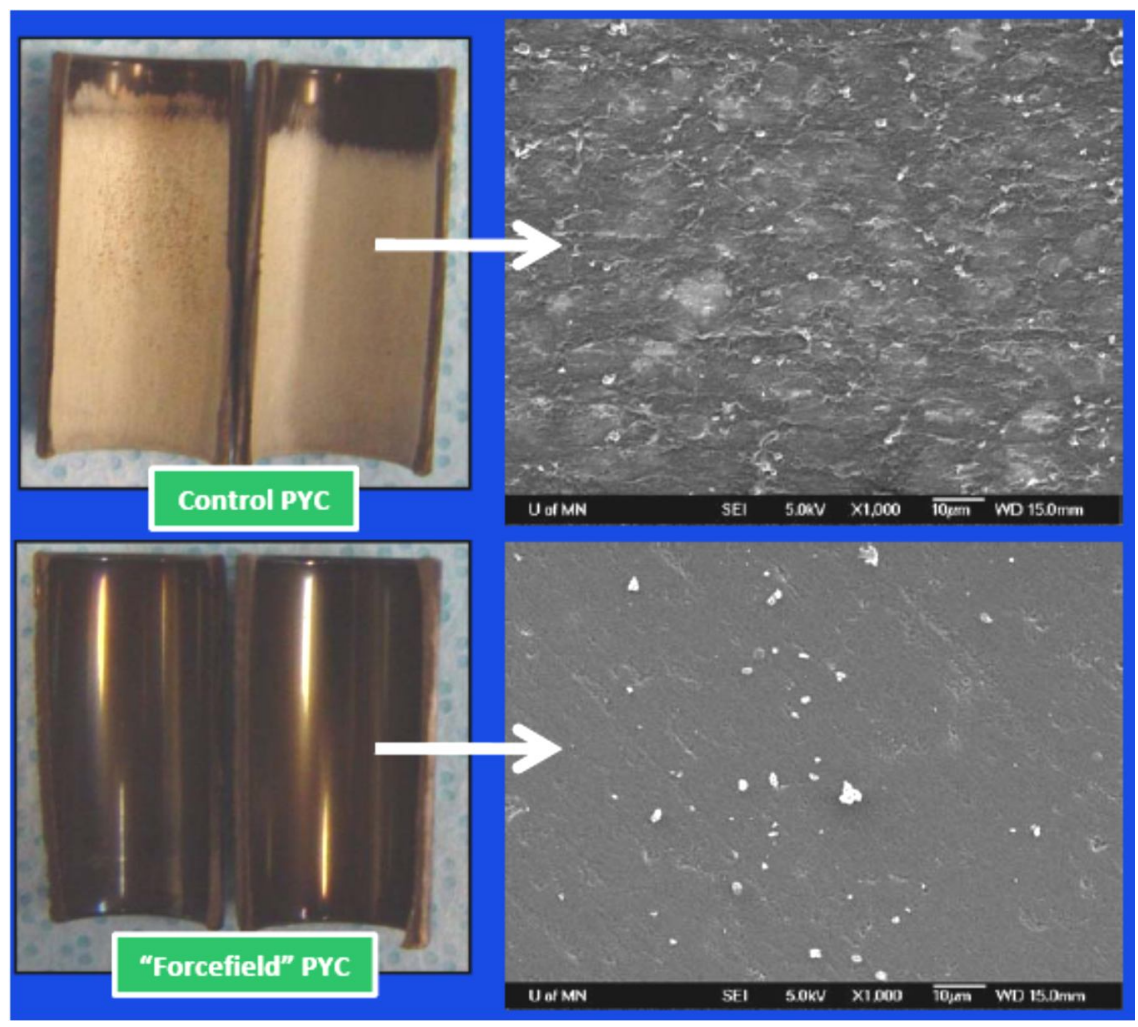

FIGURE 10 - SEM photos of platelet aggregation on "Forcefield" modified PYC and control PYC samples. The top images show clear platelet activation and spreading across the PYC while the bottom images show non-activated (round, non-attached) platelets. The 'Forcefield' technology is an important tool used to increase the biocompatibility of the cannula.

The mechanism(s) behind the non-activation of the platelets on the PYC is still an area of debate among researchers. This unique property of PYC is still not fully understood and warrants further study. For the purposes of this report this 'forcefield' surface modification was not used as it is outside the main design features being studied. However, this technology appears promising and will be incorporated into future experiments. 


\section{'Seldinger' Insertion Technique}

The current AVB technique commonly uses a $20 \mathrm{~mm}$ diameter conduit that is secured through an apical connector during bypass, or more commonly during an off bypass technique as described previously. One of the main drawbacks is the coring of the apex and use of a balloon pump to make a large enough opening to allow for suturing of the apical connector. Often this adds surgical time and risk. To overcome these obstacles, a Seldinger-like insertion technique is proposed for use with the cannula. The smaller size of the cannula allows for this type of surgical implantation.

The Seldinger technique is a medical procedure to obtain safe access to blood vessels and other hollow organs. It is named after Dr. Sven-Ivar Seldinger, who pioneered the procedure in 1953 (26). It is mainly a technique used for angioplasty, though the same principles will be used in the insertion of the cannula. The implantation will employ a small incision at the apex of the heart followed by introducers and dilators to make an opening large enough for the cannula. The cannula is then secured through use of a locking ring mechanism sutured on the heart. The main advantage of this type of technique is avoidance of coring the heart and makes rapid implantation possible. Further development of the technique and implantation tools will need to be completed in future work. 


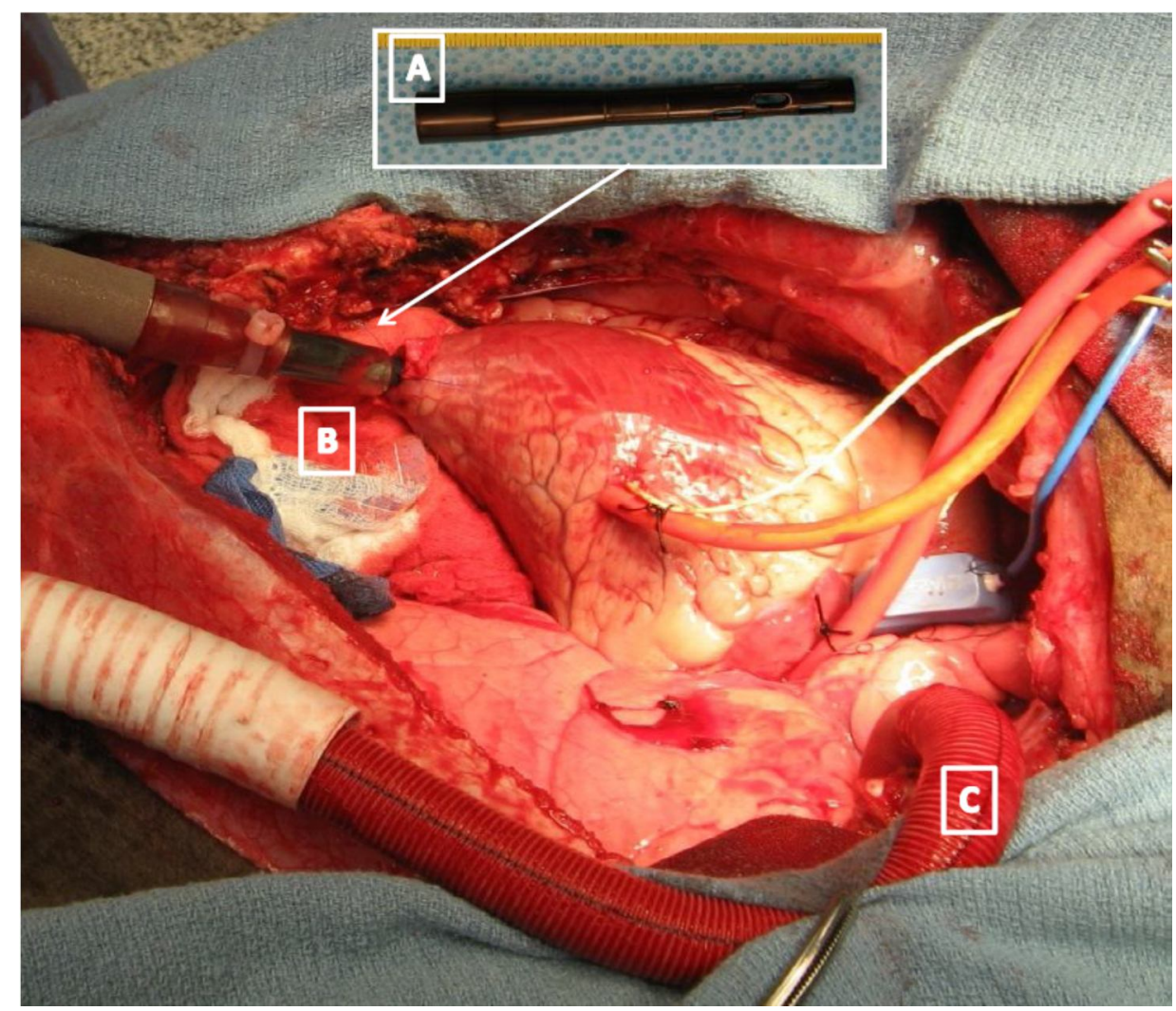

FIGURE 11 - Inflow cannula (A) designed to simplify and shorten the ventricular apical implantation procedure by using the Seldinger technique at the apex of the left ventricle (B). The conduit is then attached to the descending aorta (C). This approach eliminates the need for placing the patient on cardiopulmonary bypass, avoids ventricular coring, and achieves hemostasis through a small apical sewing ring and locking mechanism.

Figure 11 shows early, non-published work, of an implantation feasibility study by Slaughter et al. in a bovine model using the preliminary cannula prototype. This implantation uses a stab incision with rapid insertion of the cannula into the ventricle. This work showed that this type of implantation is very promising as there was minimal bleeding and complications seen at the insertion site. The cannulation system simplifies the surgical procedure and potentially minimizes morbidity/mortality to promote faster 
recovery. Anticipated benefits of this approach may include rapid application of therapy with reduced operative time and shorter hospital length of stay.

\section{EXPERIMENTAL DESIGN AND METHODS}

Three mock flow circulation and CFD analysis was performed on a series of cannulas to investigate the flow properties. The mock flow loops were a Static Flow Loop, Dynamic Flow Loop, and a Blood Trauma Loop.

\section{$\underline{\text { Static Flow Loop }}$}

The static flow mock flow loop was utilized to determine the flow versus pressure drop relationship for different size cannula under constant conditions. Seven different return venous cannulas were tested for flow characteristics using two variables, type and size of the tip. The cannulas were obtained from expired or surplus cardiopulmonary bypass equipment from surplus supplies at the Cardiovascular Innovation Institute. The aim of this experiment was to hold as many of the variables constant (pressure drop, velocity) as possible so that differences can be inferred between the cannula types. The experimental system is shown in Figure 12 and is composed of:

- Bucket Reservoir

- Flow loop composed of CPB tubing

- HeartMate II ventricle assist device (Thoratec Inc., Pleasanton, Ca)

- Small $150 \mathrm{ml}$ test section of tubing

- $2000 \mathrm{~mL}$ of Plasma-lyte (Baxter Laboratories)
- 7 various sized test cannula

- 3 Millar pressure catheters

- 1 Transonic Flow probe

- Calibration chamber

- DAQ system for data collection. 


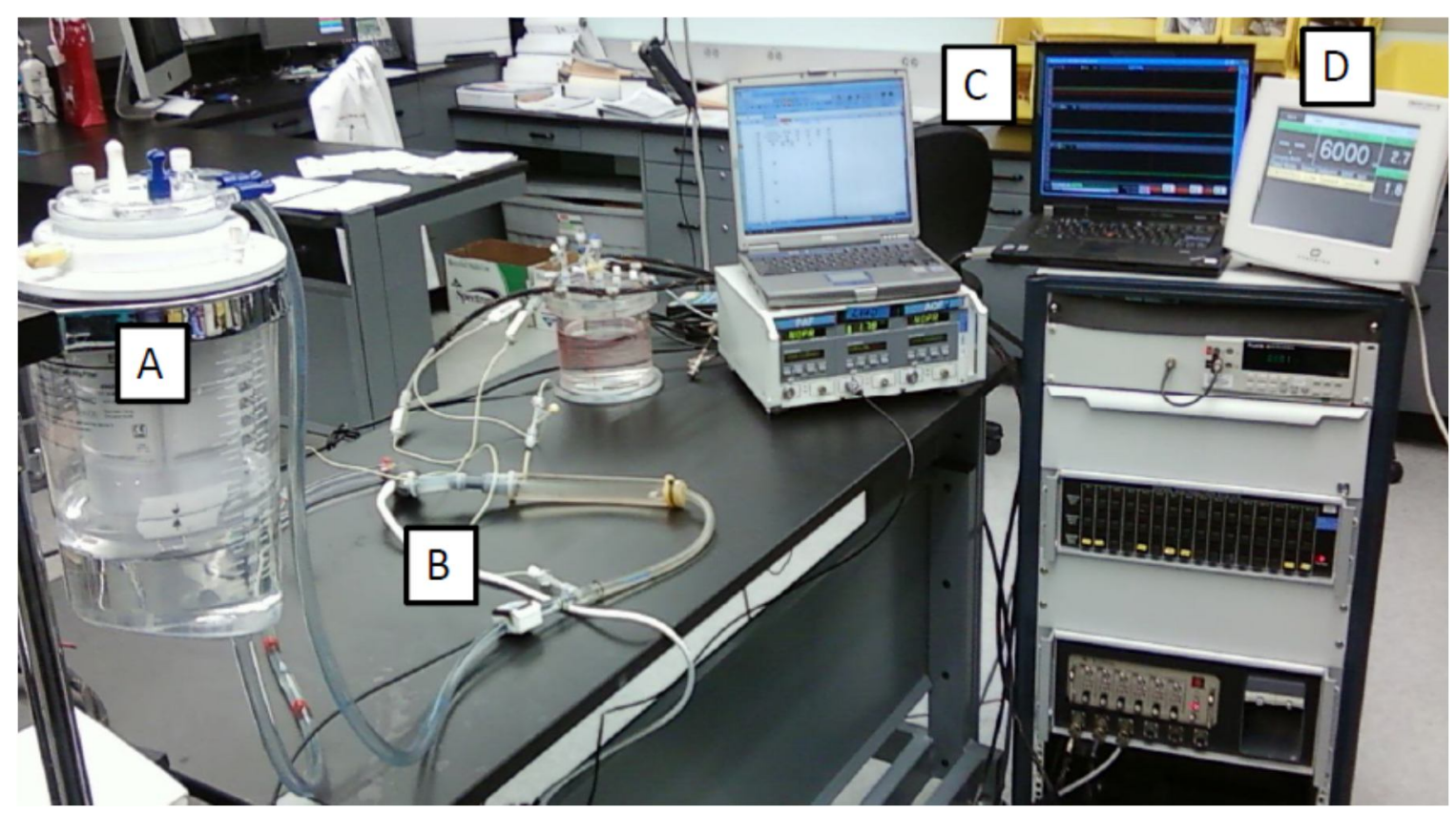

FIGURE 12 - Static Loop used for analysis of flows for different sized cannula under static and continuous pressure drop conditions composed of: A) Reservoir, B) Flow Loop C) DAQ system D) HeartMate II VAD system monitor.

While holding the pressure drop at a constant value, the flow rate was measured. Figure 13 shows the flow path of the static loop. Plasma-Lyte (Baxter Laboratories) was used for fluid flow in the static loop. Plasma-Lyte has the same viscosity as water and is a closer substitute to actual blood plasma than pure water. The tubing was standard $3 / 8 \mathrm{x}$ 3/32 class VI CPB tubing commonly used in open heart procedures (COBE Cardiovascular, Inc. Arvada, Co). The test section was composed of a small section of clear silicone tubing with an injection port for the inlet pressure catheter upstream from the test cannula. 


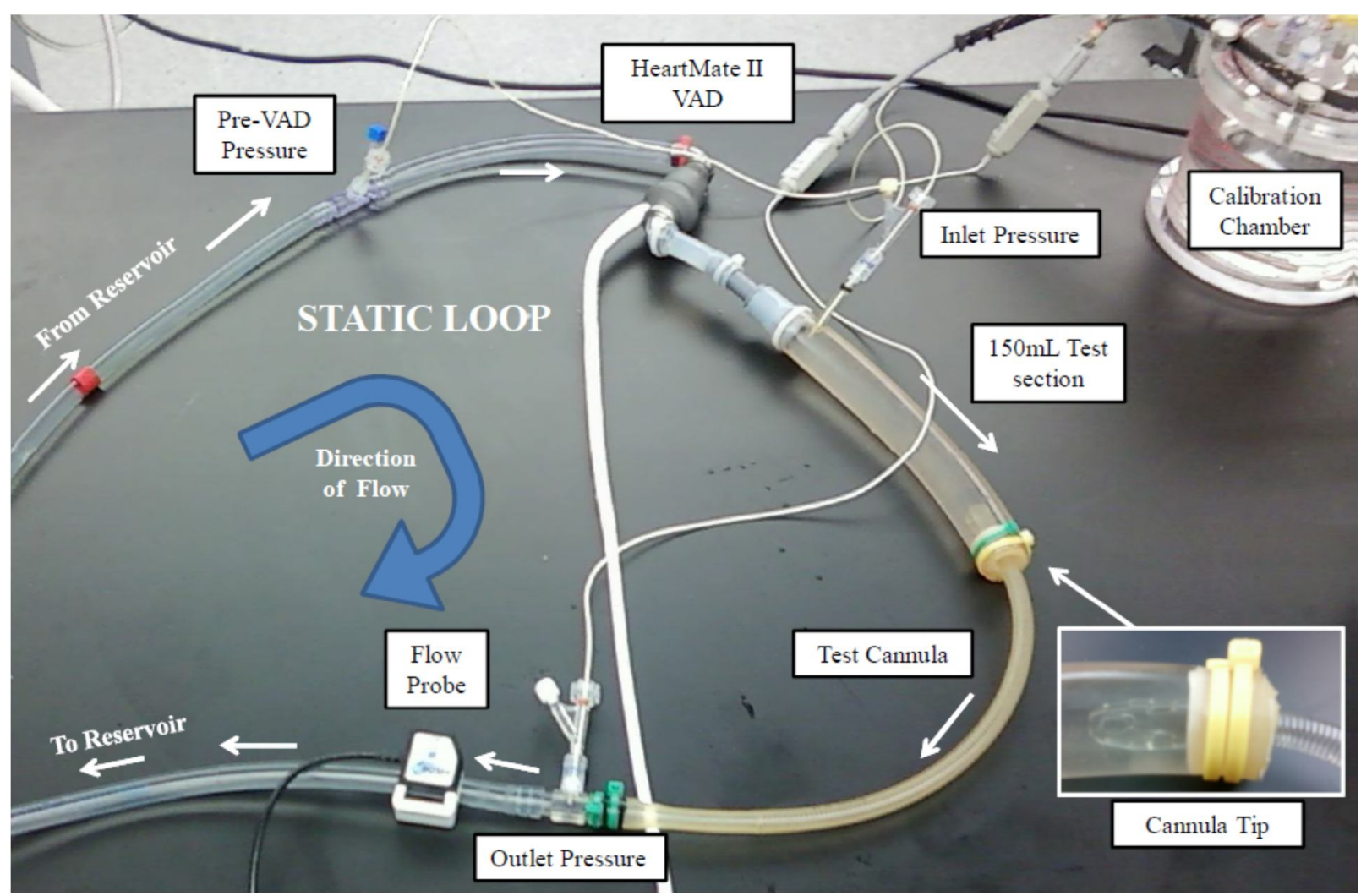

FIGURE 13 - Static Flow loop. The white arrows show the path of the flow from the reservoir to the HeartMate II through the test section and finally out back to the reservoir.

High fidelity Millar catheters were used to measure the Pre-VAD, Inlet and Outlet pressure. A Transonic flow probe was used to measure the flow rate. A calibration chamber was used to take pre and post calibration measurements.

The test catheter was inserted into the test section of the static loop so that the head of the cannula was completely surrounded by fluid as shown in Figure 13 (bottom, right). $150 \mathrm{~mL}$ was chosen for the volume of the test section as this is the approximate volume of the left ventricle at the end of the systolic period and is when the cannula will experience the highest flow rates. 
The pressure drop across the cannula was created by a small rotary pump, the HeartMate II (HMII) ventricular assist device. The only controlled variable of the experiment was the pump speed (revolutions per minute, rpm) of the HMII. In this way, the pressure drop was controlled and the flow rates for the cannula were directly compared. The pressure drop was defined as the difference between the inlet and outlet pressures measured by high-fidelity pressure catheters (Millar Instruments, Houston, TX), while the flow rate was determined by a transit-time flow probe (Transonics, Ithica, $\mathrm{NY}$ ) at the distal end of the cannula.

Each cannula was tested over the HMII operating range of 6,000 to $15,000 \mathrm{rpm}$ with pressure and flow measurements recorded at $200 \mathrm{rpm}$ increments. Five second epoch data files were collected utilizing the DAQ system at every 1,000 rpm for postprocessing and analysis. Data was collected once the static loop had reached a constant value for flow after an increment change. The return reservoir bucket was kept at the same height and the tubing components were confined to the same path for each run. After each cannula was tested the loop was filled with Plasma-Lyte back to the same volume (2,000 ml). Data was then analyzed and plotted with MATLAB and EXCEL software packages.

The resistance was obtained through a simple regression analysis. The slope of a linear model can be used as a close approximation of the true resistance. The R-Squared value was used to determine the accuracy of the resistance. This value ranges from 0 to 1 where a value of 1 represents a perfect fit. This is calculated using the following equation where $\mathrm{SS}_{\mathrm{err}}=$ sum of squares of the residuals and $\mathrm{SS}_{\mathrm{tot}}=$ the total sum of squares value.

$$
R^{2} \equiv 1-\frac{S S_{\mathrm{err}}}{S S_{\mathrm{tot}}}
$$


The cannulas ranged from 9.3 - $12 \mathrm{~mm}$ in outer diameter (28 Fr to $36 \mathrm{Fr}$ ) and were arranged into three groups based on the design features of the tip at the end of the cannula. Table IV provides the measurements taken from each cannula as well as the manufacturer of each. The surface area of the opening was approximated and measured using calipers. The cross sectional area was calculated by measurement of the inner radius of the cannula. Figure 14 shows the three types of tips that were classified based from the distinguishing characteristics as Cage, Open, or Dome.

TABLE IV

CHARACTERISTICS OF CANNULA TESTED

\begin{tabular}{|c|c|c|c|c|c|}
\hline Type & $\begin{array}{c}\text { Outer } \\
\text { Diameter Size } \\
\mathrm{mm},(\mathrm{Fr})\end{array}$ & $\begin{array}{c}\text { Inner } \\
\text { Diameter } \\
\mathrm{mm}\end{array}$ & $\begin{array}{c}\text { Cross Sectional } \\
\text { Area (CSA) } \\
\mathrm{mm} 2\end{array}$ & $\begin{array}{c}\text { Surface Area of } \\
\text { Openings (SA) } \\
\mathrm{mm}^{\wedge} 2\end{array}$ & Manufacturer \\
\hline Cage & $9.3(28)$ & 6.5 & 33.2 & 47.4 & Sarns \\
\hline Open & $9.3(28)$ & 6.7 & 35.3 & 42.0 & Research Medical Inc \\
\hline Dome & $10(30)$ & 7.4 & 43.0 & 111.0 & Terumo \\
\hline Open & $10(30)$ & 7.5 & 44.2 & 153.5 & Medtronic \\
\hline Cage & $10.7(32)$ & 7.5 & 44.2 & 69.5 & Sarns \\
\hline Dome & $11.3(34)$ & 8.8 & 60.8 & 144.9 & Sarns \\
\hline Cage & $12(36)$ & 8.8 & 60.8 & 74.0 & Sarns \\
\hline
\end{tabular}




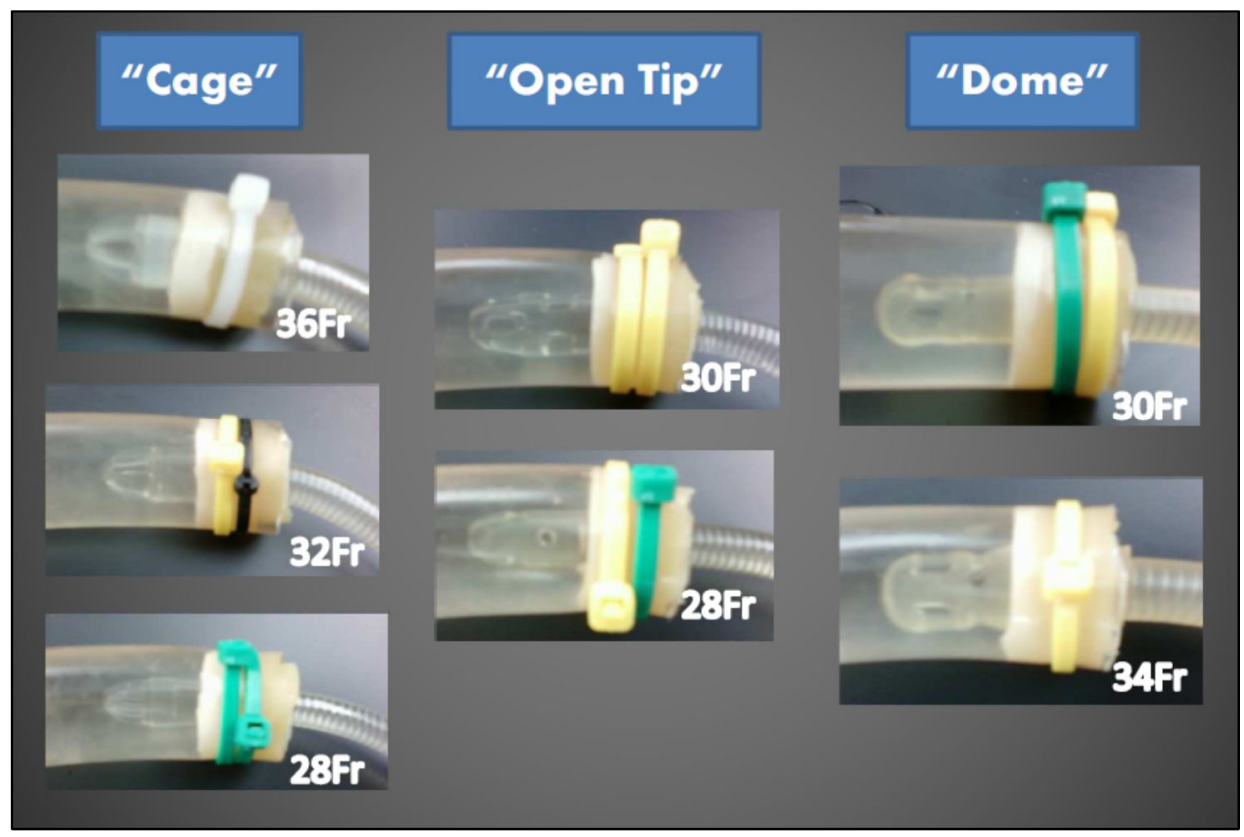

FIGURE 14 - The seven cannula used separated by type and size of tip. The Cage type is distinguished by 4 main holes at the tip formed resembling a cage. The Open type features an open orifice at the tip with eight sideholes arranged on the head of the cannula. The Dome type features a smooth dome tip with eight sideholes arranged around the head of the cannula.

\section{Blood Trauma Loop}

To test for possible trauma and damage to blood cells caused by high shear rates in the cannula, a static flow test using bovine blood was used. The preliminary prototype cannula was tested in a mock loop with a continuous flow pump (HeartMate II). The circuit consisted of a $500 \mathrm{ml}$ reservoir, $23 \mathrm{~cm}$ of standard PVC uncoated tubing, 40 micron inline filter, transonic flow probes and pressure transducers as seen in Figure 15. 


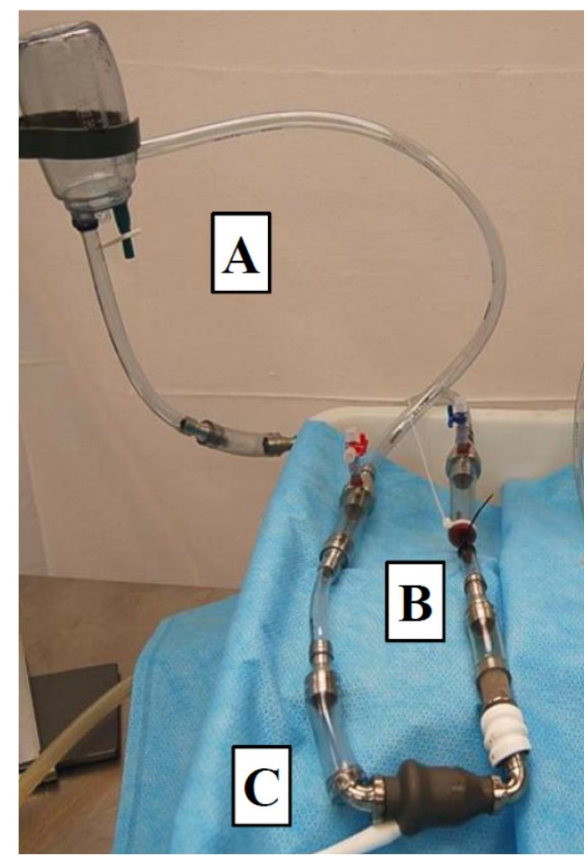

FIGURE 15 - Flow loop used in blood trauma data. A) 500mL reservoir, B) Testing section, C) HeartMate II continuous flow VAD.

Fresh whole bovine animal blood was collected and stored for less than 48 hours for performance testing. To minimize the effects of blood variation for tests run with the cannula, a sufficient amount of blood (5-6 liters) was drawn from a single animal to permit testing. Blood hemolysis testing followed ASTM international standard F1830 (27). Data collection consisted of blood hematology: white blood cell, red blood cell, platelet, hemoglobin, hematocrit, and plasma free hemoglobin (WBC, RBC, PLT, HgB, HCT, PF-HgB). Pump performance (rpm's and flow rates) and mock circuit status (pressure inlet/outlet, delta and measured flow rates) were also taken.

The testing conditions were held for at a constant range with the pump operating at $8400 \mathrm{rpm}$ for the length of the experiment. These test conditions created a pressure drop of $40 \mathrm{mmHg}$ with a constant flow rate of $2.4 \mathrm{lpm}$. Baseline readings were recorded 
and samples were taken at intervals of 90 minutes for a total of 6 hours. Pressure and flow measurements were taken from the pressure catheters and Transonic flow probes in the flow loop. Complete blood chemistry profiles and blood gases were measured with Gem 4000 (Instrumentation Laboratory, Warrington, UK). Cell counts were measured with HemaVet 950FS (Drew Scientific, Inc., Dallas, TX). Plasma free hemoglobin was measured using a HemoCue Plasma/Low Hb Photometer (HemoCue, Inc., Lake Forest, CA) shown in Figure 16.

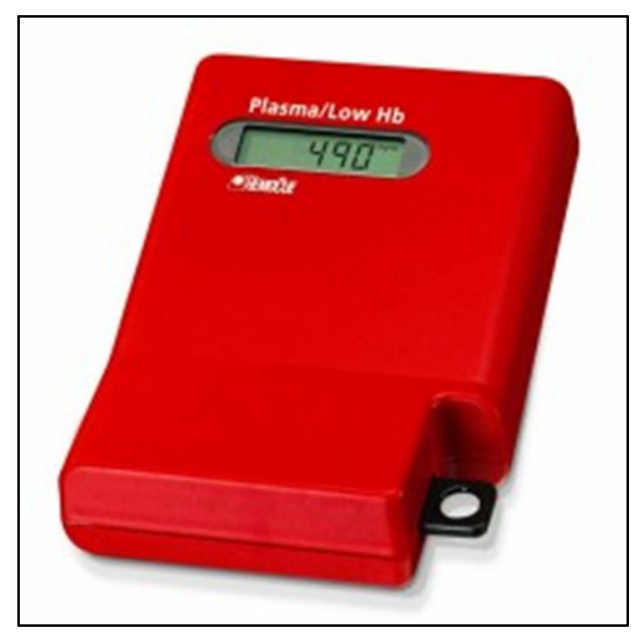

FIGURE 16 - HemoCue Plama/Low Hb Photometer used in the trauma study to measure plasma free hemoglobin.

In compliance with clinical guidelines for VADs (FDA and ISO 7199), blood was anticoagulated with heparin (4500 units per liter of blood) (28). A minimum Activated Clotting Time (ACT) of 300s was maintained with additional heparin as needed. To simulate dilution during bypass, the blood was diluted with normal saline to a hematocrit of $25 \pm 3 \%$ for the testing.

Figure 17, Left shows the in vitro flow loop used for the preliminary prototype; on the Right, the preliminary prototype is inserted into a small silicone o-ring that models 
the Seldinger implantation technique that uses a stab wound to introduce the cannula into the apex of the left ventricle. Similar to the static flow mock loop experiment, this study used the HeartMate II continuous flow VAD, in this case set at a constant speed of 8400 rpm.

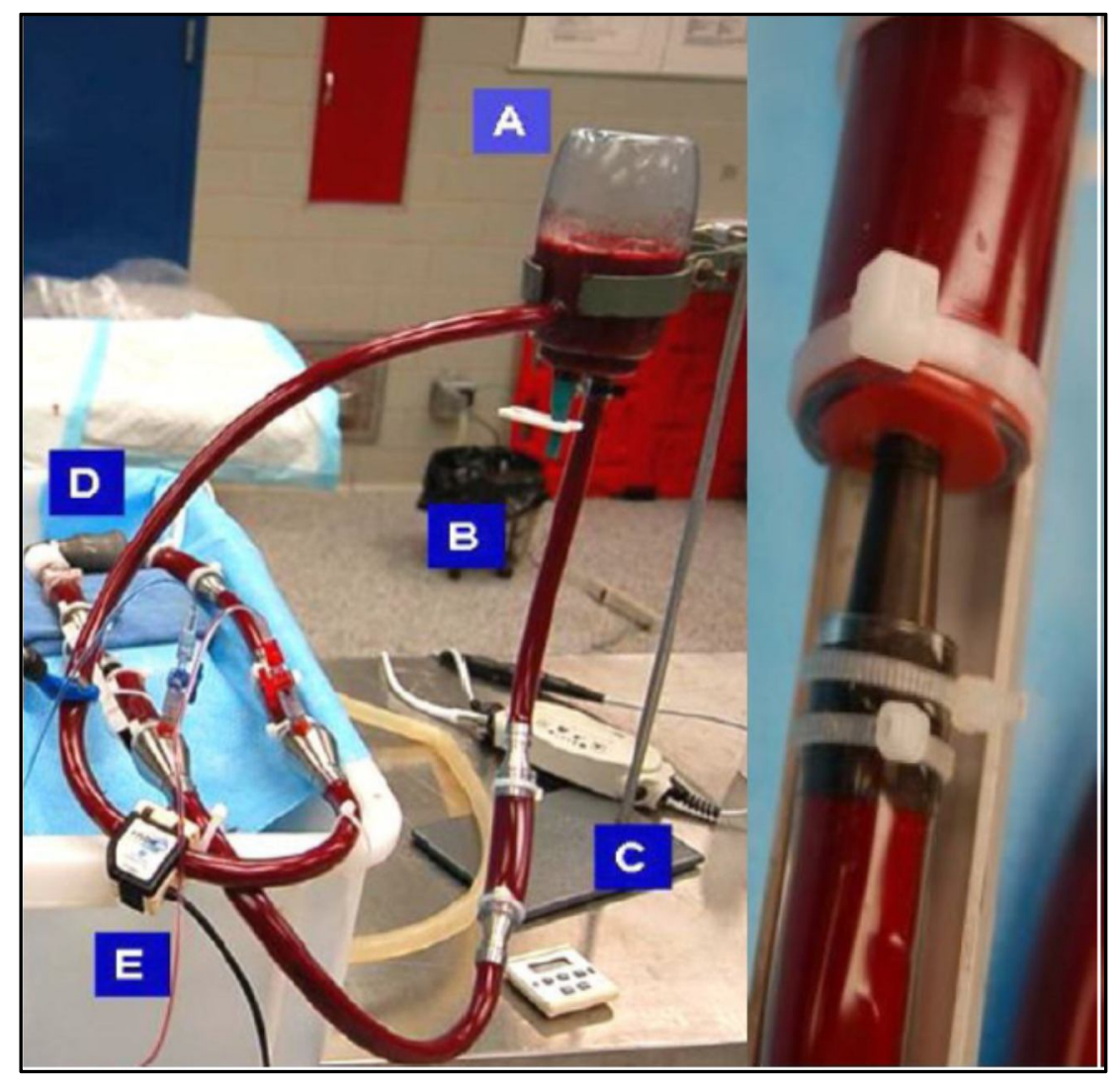

FIGURE 17 - LEFT: Bovine blood flowing through a mock flow loop consisting of A) 500mL Reservior, B) Standard 23cm CPB tubing, C) HeartMate II Controller, D) HeartMate II continuous flow VAD, E) Transonic Flow Probe and Pressure Catheter inside injection port. RIGHT: Preliminary Prototype inserted inside the tubing simulating the insertion technique used for implantation. 
The flow rate was kept at a constant 2.4 lpm over the course of the 6 hour study. Heparin was administered throughout the course of the study to maintain a constant ACT greater than 300 seconds. Also blood samples were taken from the circuit every 90 minutes for blood analysis and heparin was added throughout the study period which has a slight dilution effect.

\section{Dynamic Flow Loop}

The dynamic flow loop study was used to investigate the flow characteristics of different size cannula under simulated physiologic pressure and flow conditions. A mock flow loop of the adult systemic circulation was used to test the AVB system. Three cannula were tested: 1) Prototype design, 2) 32Fr Sarns Venous Return cannula, and 3) 36Fr Sarns Venous Return cannula. The tip design of the three cannulas are very similar (open distal orifice with sideholes along body of the tip) so the effect of cannula size on physiologic conditions was the goal of this study.

The test conditions included a baseline with the AVB system clamped off from the loop, the AVB system under normal physiologic conditions, and the AVB system under three different aortic stenotic conditions (mild, moderate, and severe). High fidelity pressure catheters (Millar Instruments, Houston, TX) and transit-time flow probes (Transonics, Ithica, NY) were used measure simulated cardiovascular pressures and flows. For each test condition, 15 second data files were recorded using the DAQ system.

Each cannula was inserted into the apical cannulation port at the apex of the mock ventricle. The cannula was connected to a $19 \mathrm{~mm}$ graft and mechanical valve conduit, 
which was connected to a T-junction on the mock descending aorta that completed the AVB circuit. Glycerol was used for a blood analog with a viscosity of 3.7 centipoise, similar to that of blood. The flow loop was instrumented to record the following hemodynamic parameters:

- Aortic Flow (AoF)

- Aortic Pressure (AoP)

- Left Ventricular Pressure (LVP)

- Left Atrial Pressure (LAP)

- AVB conduit Flow (GRF)

- Pressure inside the AVB conduit (GRP)

Aortic stenosis was simulated in the mock loop by applying a clamp to the mock aortic root. Baseline data was taken where the AVB conduit was clamped off from the mock loop at the apex and the descending aorta. With the AVB conduit unclamped, four different AVB configurations were tested: 1) No aortic stenosis under normal physiological conditions, 2) Mild aortic stenosis, 3) Moderate aortic stenosis, 4) Severe aortic stenosis. The peak-to-peak pressure difference (peak LVP - peak AoP) was used to quantify the pressure drop across the valve and assign the degree of aortic stenosis. Table IV provides the testing conditions used for this study (Table V, Left) and the transvalvular pressure differences used to simulate aortic stenosis (Table V, Right) as outlined ACC/AHA Practice Guidelines. 
TABLE V

TESTING CONDITIONS FOR THE DYNAMIC FLOW STUDY.

\begin{tabular}{|l|c|c|c|}
\hline \multicolumn{2}{|l|}{ Mock Parameter Values } & \multicolumn{2}{c|}{ Aortic Stenosis Conditions } \\
\multicolumn{2}{|c|}{ Testing Parameters } & \multicolumn{2}{c|}{ Valve Gradient } \\
\hline Mean AoP & $95 \mathrm{mmHg}$ \\
\hline CO & $5.0 \mathrm{~L} / \mathrm{min}$ \\
\hline LVPed & $2-5 \mathrm{mmHg}$ \\
\hline DLP & $210 \mathrm{mmHg}$ & $0 \mathrm{mmHg}$ \\
\hline
\end{tabular}

AoP, aortic pressure; CO, cardiac output; LVPed, left ventricular end diastolic pressure; DLP, drive line pressure

The experiments were completed at the Cardiovascular Innovation Institute. The mock loop was tuned to the testing parameters through adjustment of the vascular resistance and compliance. The degree of aortic stenosis was controlled through adjustment of a resistance clamp at the aortic root. After the loop had reached a stable condition, a 15 second data file was taken on the DAQ system. The testing protocol for cannulas tested was as follows.

1. A baseline reading was taken with the AVB circuit clamped off from the mock loop, which had been tuned to the testing conditions listed in Table V.

2. The resistance clamp at the aortic root was adjusted until the transvalvular pressure difference matched the desired value (none, mild, moderate, or severe) and a baseline reading was taken.

3. The AVB circuit was unclamped and a data file was taken after the system had reached stability.

4. Steps 2 and 3 were repeated until all four of the AVB configurations were completed 
5. The aortic root was then fully occluded with all flow going through the AVB conduit and a data file was taken.

6. A post calibration file was taken with a static circuit where the pump was turned off and all clamps removed.

7. The cannula was removed from the circuit and the next cannula was placed in the circuit for testing.

Table VI provides the size of the cannulas, valve, and graph used as well as photos of the tips of the cannula. The venous cannula used had 'open' tip shape with an open orifice at the distal tip and eight sideholes along the body of the tip.

\section{TABLE VI}

\section{CANNULA CHARACTERISTICS}

\begin{tabular}{lccc} 
Cannula & $\begin{array}{c}\text { Tip Size } \\
(\mathrm{mm})\end{array}$ & $\begin{array}{c}\text { Valve Size } \\
(\mathrm{mm})\end{array}$ & $\begin{array}{c}\text { Conduit Size } \\
(\mathrm{mm})\end{array}$ \\
$\begin{array}{l}\text { Preliminary Prototype } \\
\text { Sarns Malleable Venous }\end{array}$ & 10 & 19 & 19 \\
$\begin{array}{l}\text { Return Catheter 32Fr } \\
\text { Sarns Malleable Venous }\end{array}$ & 10.7 & 19 & 19 \\
Return Catheter 36Fr & 12 & 19 & 19 \\
\hline
\end{tabular}


The data files from the study were first analyzed in using the HEART program using MATLAB. Each file was picked on a beat-to-beat basis and systolic pressure peaks were chosen from the plots. Mean aortic flow, distal aortic flow, conduit flow, aortic pressure, conduit pressure, left ventricular pressure, and left atrial pressure (AoF, AoFd, GRF, AoP, GRP, LVP, LAP; respectfully) values were calculated. The valve gradient was calculated from the peak AoP and LVP pressures. Total \% Conduit flow was calculated using the AoF, Aofd, and GRF. Appendix II provides the data obtained and two sample plots that were beat picked. A total of 37 data files were collected.

\section{Computation Flow Dynamic (CFD) Analysis}

Computational flow analysis was used to investigate the effects of design features of the cannula tip, specifically the number and size of the sideholes on the cannula. The wall shear stresses, shear strain rate, and flow rate were calculated through simulation in

the ANSYS CFD (ANSYS Inc., Canonsburg, PA, U.S.A.) software package. Differences in these parameters between cannula designs were used to evaluate flow characteristics. The CFD analysis was broken into two main studies: Number of Sideholes and Size of Sideholes.

The CAD models were created using SolidWorks (SolidWorks SP3.0, Dassault Systèmes; Vélizy, France) CAD software and are similar in design to the preliminary prototype. A larger view of a typical CAD model for both the cannula and the mesh model can be seen in Appendix III. The cannulas created for the CFD analysis were all $12 \mathrm{~mm}$ in diameter and identical to each other with the exception of the number and size 
of the sideholes. Through this method, the characteristics of the sideholes design could be directly studied.

The CAD files of the cannula designs were loaded into ANSYS workbench project and the 3D models were checked for continuity. The model was then meshed using the AutoMesh function (Figure 18) with the appropriate settings for fluid flow. A fine relevance center sizing was used to ensure ample node and element creation around those parts of the geometry which have finer detail. The meshes were visually verified for the proper geometry. Mesh statistics for each cannula are presented in Tables VIII and IX in the following sections.

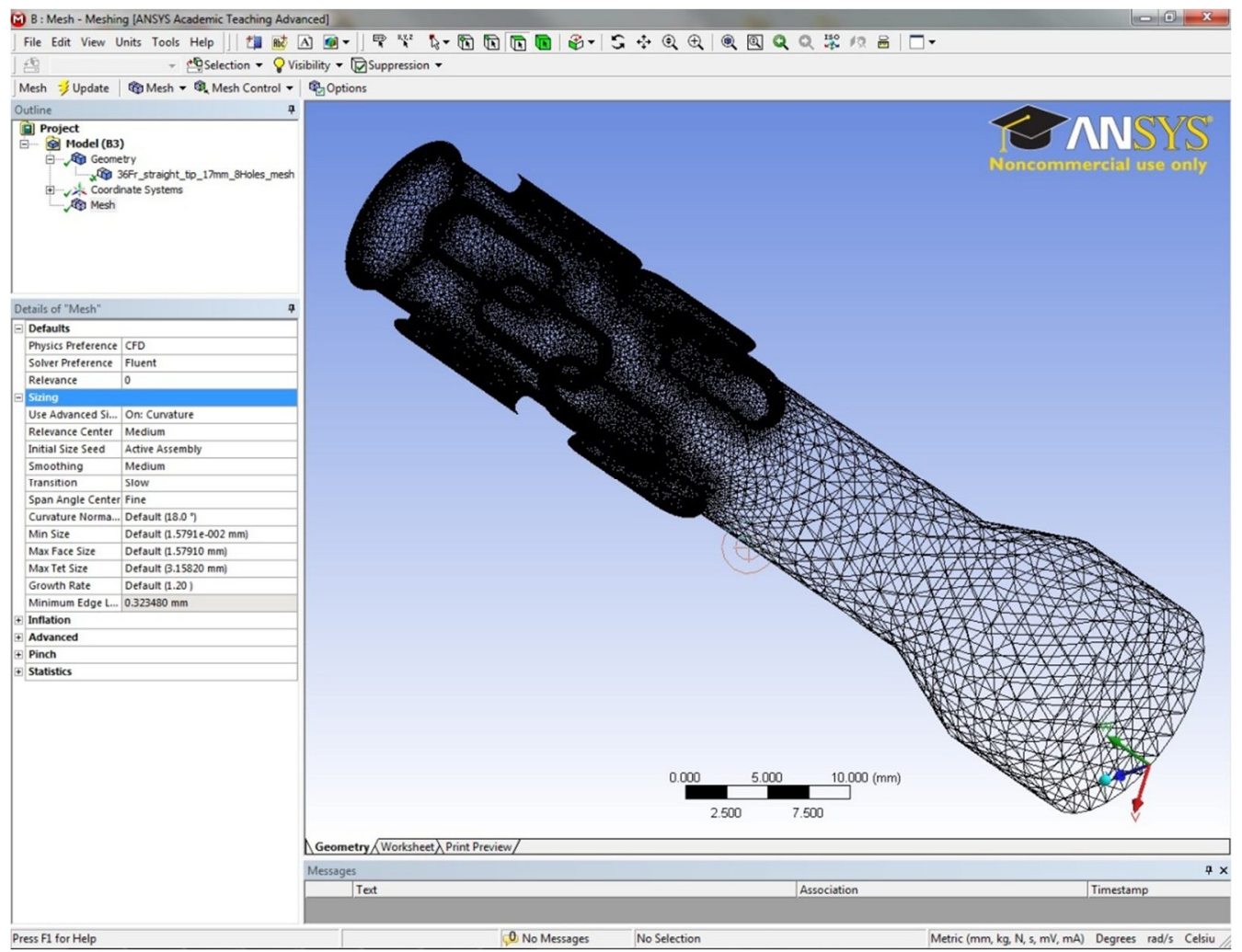

FIGURE 18 - The meshing program for ANSYS CFD software analysis. A medium density mesh was chosen for this analysis. 
Pre-solver software was used to define boundary regions and initial conditions.

Figure 19 shows the defining of the boundary regions and is used to set up the running parameters. A cylinder (radius $=15 \mathrm{~mm}$, length $=50 \mathrm{~mm}$ ) was placed around the cannula body was used for the inlet boundary region. This allows for development of flow into the cannula and will allow for more accurate streamlines and vector fields around the cannula orifices.

The cylinder is placed only around the areas of the cannula that will be inside the left ventricle and models in vivo-like conditions. The inlet boundary allows for flow to originate from all sides of the cannula. In this way the wall shear stress and strain can be measured for all blood contacting portions of the cannula.

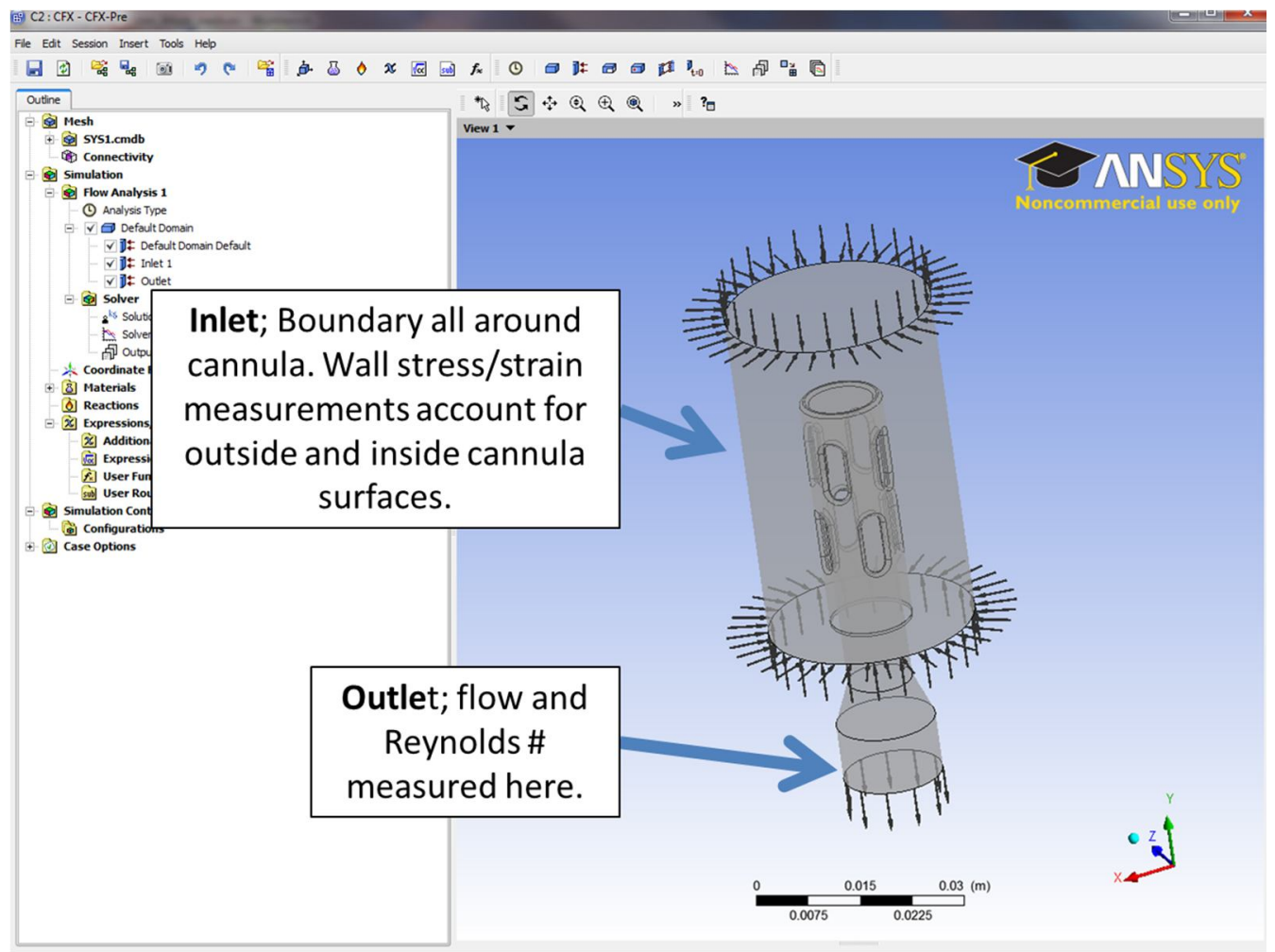

FIGURE 19 - Defining the boundary regions and initial conditions for the cannula in the Pre-solver component software. 
Simulation operating conditions are presented in Table VII. A constant pressure boundary condition with a pressure difference between inlet and outlet of $6 \mathrm{mmHg}$ was assumed. The operating fluid, blood, was assumed to be a Newtonian fluid with a density of $\rho=1.1 \mathrm{~g} / \mathrm{cm}^{3}$ and viscosity $v=0.035$ Poise. Flow was not assumed to be laminar as a $\mathrm{k}-\varepsilon$ turbulence model was used.

\begin{tabular}{lc}
\multicolumn{2}{c}{ TABLE VII } \\
SIMULATION OPERATING CONDITIONS \\
\begin{tabular}{lc} 
CFD Simulation Conditions \\
\hline Fluid Type & Newtonian \\
Density & $1.1 \mathrm{~g} / \mathrm{cm}^{\wedge} 3$ \\
Viscosity & $0.035 \mathrm{P}$ \\
Turbulence Model & k epsilon \\
Boundary Condition & Constant Pressure \\
Pressure Gradient & $6 \mathrm{mmHg}$ \\
\hline
\end{tabular}
\end{tabular}

The constant $6 \mathrm{mmHg}$ pressure boundary represents the peak systolic transconduit pressure gradient and is based from clinical hemodynamic data (29). Vliek et al. reported hemodynamic data for AVB intraoperative surgery with a median peak gradient between the left ventricle and distal conduit in 18 patients ( 2 with 16-mm apical connectors, 6 with 14-mm apical connectors, and 12 patients with 12-mm apical connectors) as measured directly in the operating room of $5.6+/-3.8 \mathrm{mmHg}$ (range, 0 to $14 \mathrm{mmHg}$. 
Examination of the Reynolds number is an important factor in CFD analysis and was measured at the cannula outlet. This is a dimensionless number that gives a measure of the ratio of inertial forces to viscous forces defined as $\mathrm{Re}=\mathrm{U} * \mathrm{D} / v$ (where $\mathrm{U}$ is the average velocity through the orifice, $\mathrm{D}$ is the orifice diameter, and $v$ is the average kinematic viscosity of blood). Application of this number though is also dependent on the type of opening. Threshold Reynolds number for turbulent flow is markedly lower (approximately 800) for flow through an orifice, whereas it is higher (around 3,000) for a pipe (in this case, a conduit).

The CFX solver ran until the solving equations converged to a final solution. The post-solver component was then used to analyze and visualize the results. Stream lines, gradient profiles, vector fields, and slice planes were used for visual analysis while average, peak, and minimum values for various parameters such as flow rate, pressure, shear rate, and velocity at the boundary regions were calculated. A custom results report was generated to obtain desired statistics, figures, and data from the simulations. An example of the one of these reports can be seen in Appendix IV.

\section{Number of Sideholes Study}

This study compared four different $12 \mathrm{~mm}$ diameter (36Fr) cannulas for differences in flow characteristics based on the number of sideholes on the tip of the cannula. This group was comprised of cannula with: No sideholes, 4 sideholes, 8 sideholes, and 12 sideholes. Figure 20 gives the CAD models of each of the four models as well as the model used for meshing and CFD analysis. The cannulas were identical to each other with only differences being in the number of sideholes each one featured. 


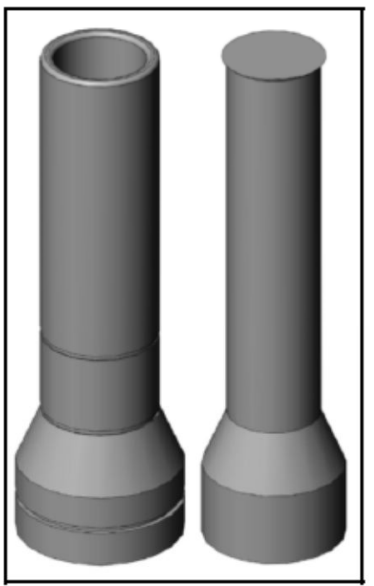

No Sideholes

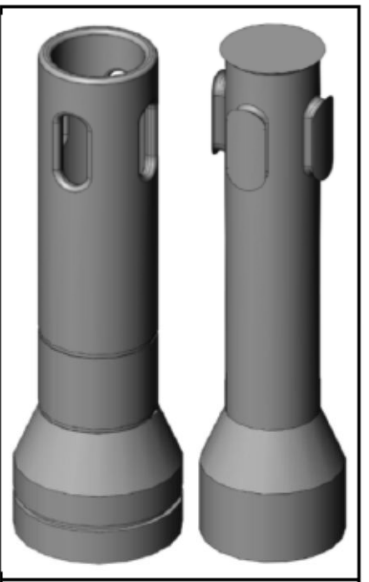

Four

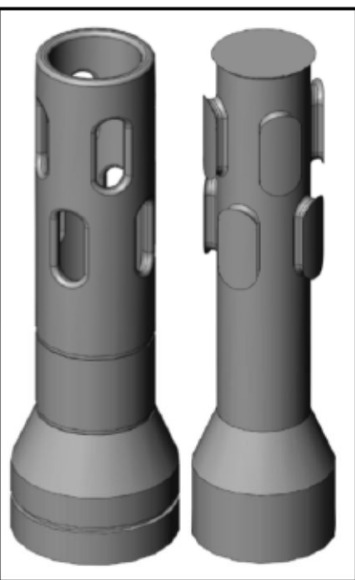

Eight

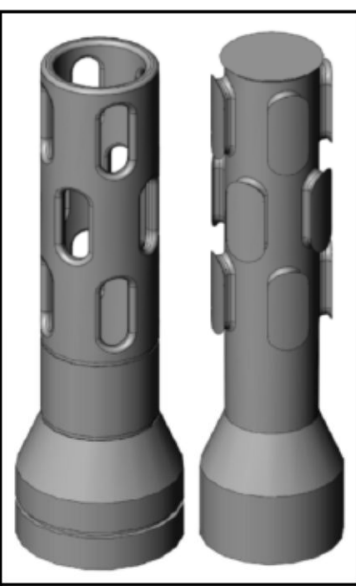

Twelve

FIGURE 20 - The CAD models for the number of sideholes CFD study. Both the cannula design (left) and the model used for meshing and CFD analysis (right) are shown for the four different models.

The sideholes on all the cannula in this study were the same size, having an oval shape with a radius of $3 \mathrm{~mm}$ and an area of $46.6 \mathrm{~mm}^{2}$. This shape and size was chosen as it is similar to sidehole designs used in the cannulas studied in the mock flow loop experiments. Table VIII provides the mesh characteristics, surface area, and volume for each cannula.

TABLE VIII

MESH CHARACTERISTICS.

Number of Sideholes

\begin{tabular}{lcccc} 
& None & Four & Eight & Twelve \\
\hline Nodes & 68346 & 149169 & 226986 & 297412 \\
Elements & 370144 & 814855 & 1241999 & 1629111 \\
Area (m^2) & $3.34 \mathrm{E}-03$ & $3.21 \mathrm{E}-03$ & $3.08 \mathrm{E}-03$ & $2.95 \mathrm{E}-03$ \\
Volume (m^3) & $3.65 \mathrm{E}-05$ & $3.67 \mathrm{E}-05$ & $3.68 \mathrm{E}-05$ & $3.70 \mathrm{E}-05$ \\
\hline
\end{tabular}




\section{Size of Sidehole Study}

Similar to the number study, the objective of the size study was to determine differences between $12 \mathrm{~mm}$ diameter (36Fr) cannula that have different sized sideholes. Four cannulas with eight sideholes of varying sizes were tested. Figure 21 shows the cannulas tested which featured small, medium, and large sideholes of the same shape. A fourth cannula featured the same size sideholes as the medium cannula but did not have the $0.5 \mathrm{~mm}$ fillet used on all the edges of the openings. This cannula was used to compare the effect that fillet geometry had on flow.

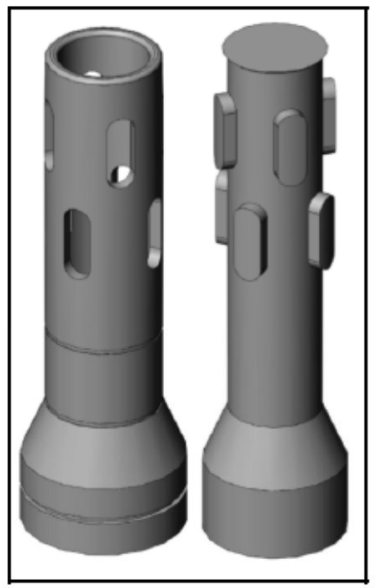

No Fillet

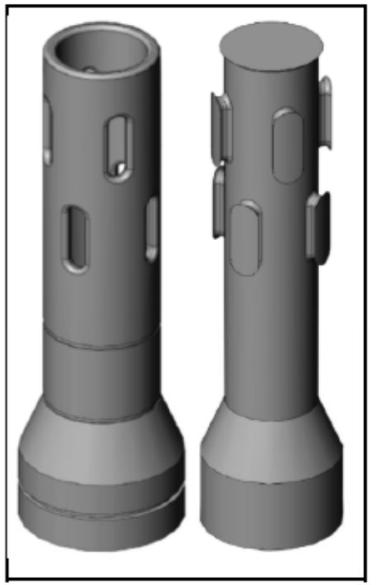

Small

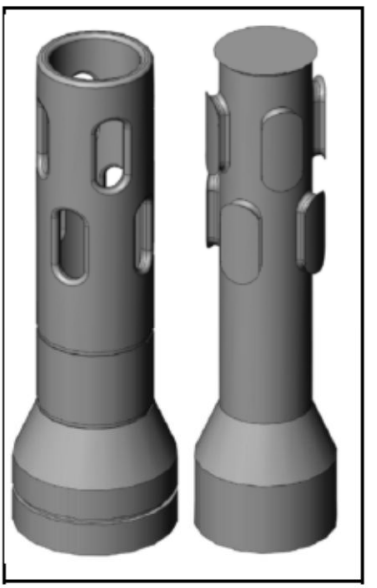

Medium

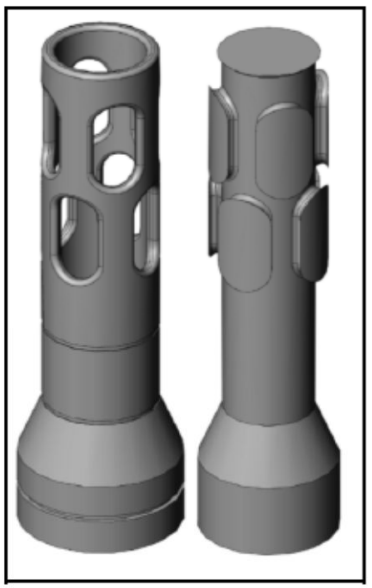

Large

FIGURE 21 - The CAD models for the size of sideholes CFD study. Both the cannula design (left) and the model used for meshing and CFD analysis (right) are shown for the four different models.

The medium size cannula features the same size sideholes that were tested in the number of sideholes study. The large size cannula had sideholes that were twice the area of the medium, while the small cannula had sideholes that were half the area of the 
medium cannula. This provided a nice range of sizes for comparison purposes. The sizes of the sideholes are presented in Table IX. The radius of the oval shape and total area are given, as well as the mesh characteristics, surface area, and volume for each cannula.

TABLE IX

AREA OF SIDEHOLES AND MESH CHARACTERISTICS

\begin{tabular}{lcccc} 
& \multicolumn{4}{c}{ Size of Sideholes } \\
& Small & Medium & Large & No Fillet \\
\hline Radius (mm) & 1.92 & 3 & 4.56 & 3 \\
Sidehole Area (mm^2) & 23.13 & 46.26 & 92.52 & 46.26 \\
Nodes & 200796 & 226986 & 257983 & 82636 \\
Elements & 1095191 & 1241999 & 1417062 & 448047 \\
Area (m^2) & $3.24 \mathrm{E}-03$ & $3.08 \mathrm{E}-03$ & $2.80 \mathrm{E}-03$ & $3.17 \mathrm{E}-03$ \\
Volume $\left(\mathbf{m}^{\wedge} \mathbf{3}\right)$ & $3.67 \mathrm{E}-05$ & $3.68 \mathrm{E}-05$ & $3.70 \mathrm{E}-05$ & $3.68 \mathrm{E}-05$ \\
\hline
\end{tabular}

\section{INSTRUMENTATION AND EQUIPMENT}

Experiments were performed at Cardiovascular Innovation Institute on the University of Louisville medical campus in Louisville, KY and the Biologic Resources Laboratory at the University of Illinois at Chicago. Experiments were carried out with the assistance of Cary Woolard and Michael Sobieski under the supervision of Dr. Steven Koenig and Dr. Guruprasad Giridharan. The following equipment and materials was used in the design, testing, and analysis of the cannula: 
- Data Acquisition System

- Measurement Instrumentation

- Millar high fidelity pressure catheter

- Transonic Flow probes

- Instrumentation Rack

- Data Acquisition Software

- LabVIEW (National Instruments, Austin, TX)

- Mock Flow Apparatus

○ Continuous Flow

- Static Flow Loop

- Blood Trauma Study

- Dynamic Flow

- Adult Circulation Mock Flow Loop

- Computer Software Packages

- Data Analysis

- MATLAB (The MathWorks, Inc., Natick, MA)

- Microsoft Excel

- Computer Aided Design (CAD)

- SolidWorks (SP3.0 2009, Dassault Systems)

- AutoCAD (AutoCAD 2008, San Rafael, CA)

- Computational Fluid Dynamic (CFD)

- ANSYS CFX (ANSYS Inc., Canonsburg, PA, U.S.A.)

\section{Data Acquisition System (DAQ)}

Pressure catheters, flow probes, and the DAQ system was used to measure, visualize, and store data collected from mock flow loop experiments completed. The DAQ system was used for all experiments to test the design of both the initial and final prototypes.

\section{Measurement Instrumentation}

Two different types of pressure catheters and flow probes were used in the flow experiments performed. A high fidelity, dual pressure-volume conductance catheter Millar Instruments, Houston, TX) was used for simultaneous mock ventricular pressure, 
ventricular volume, and aortic pressure measurements in the dynamic loop. Single tip, high fidelity catheters (Millar Instruments, Houston, TX) were inserted into introducer ports for measuring pressures in both static and dynamic flow loops. Flow was measured with clamp and in-line, transit time flow probes (Transonics, Ithaca, NY) were also used in both experiments. Pressure and flow transducers were calibrated before and after to verify gain and offset accuracy.

\section{Instrumentation Rack}

The instrumentation rack was composed of transducers, amplifiers, and signal processors. This custom built clinically approved Good Laboratory Practices (GLP) compliant DAQ rack is described in Koenig et al. (30) and was used to collect and store data from the mock loop studies shown in Figure 22. Signal conditioning was completed using transducer amplifiers (Ectron, San Diego, CA), a transit time flowmeter (Transonics, Ithaca, NY), a volume conductance unit (Leycom, Sigma V, Netherlands), and other peripheral conditioners integrated into the instrumentation system. Signal conditioned data were low pass filtered at $60 \mathrm{~Hz}$, analog to digitally converted (AT-MIO16E-10 and LabVIEW, National Instruments, Austin, TX) at a sampling rate of $400 \mathrm{~Hz}$, and stored on a personal computer (7). 


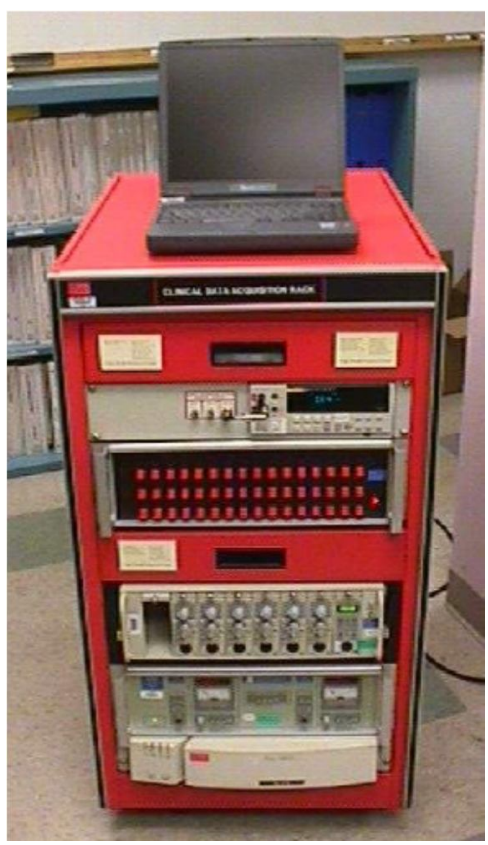

FIGURE 22 - DAQ system used to collect and store data from the mock flow loop studies used to characterize the cannula (30).

This DAQ system allows the user to connect a variety of instrumentation and then condition, amplify, and process the signals. A 16-channel signal conditioning and distribution unit is used to drive multiple peripheral monitoring and recording devices. The DAQ system has fixed gain and offset control to maximize A/D input range of peripheral devices, and low-pass filters to remove electrical noise and prevent aliasing. An analog to digital converter then allows for data storage. Signals from pressure catheters and flow probes are amplified and conditioned and are seen real-time on the monitor. Further detail concerning the components, construction, and operation of the DAQ unit is outlined in Koenig et al. (30).

The software used to for data acquisition is also a custom built LabVIEW (National Instruments, Austin, TX) described by Drew et al. $(31,32)$. This graphical 
oriented program gives users a plethora of tools to aide in the capture and analysis of data. The Cardiovascular Data Acquistion Software (CDAS) program developed by this group allows for custom setup for the 16 channel DAQ system. After the user has set up the desired profile, the program is run and allows for real-time display of waveforms collected from experiments. Figure 23 shows a layout of real-time waveform collection and GUI.

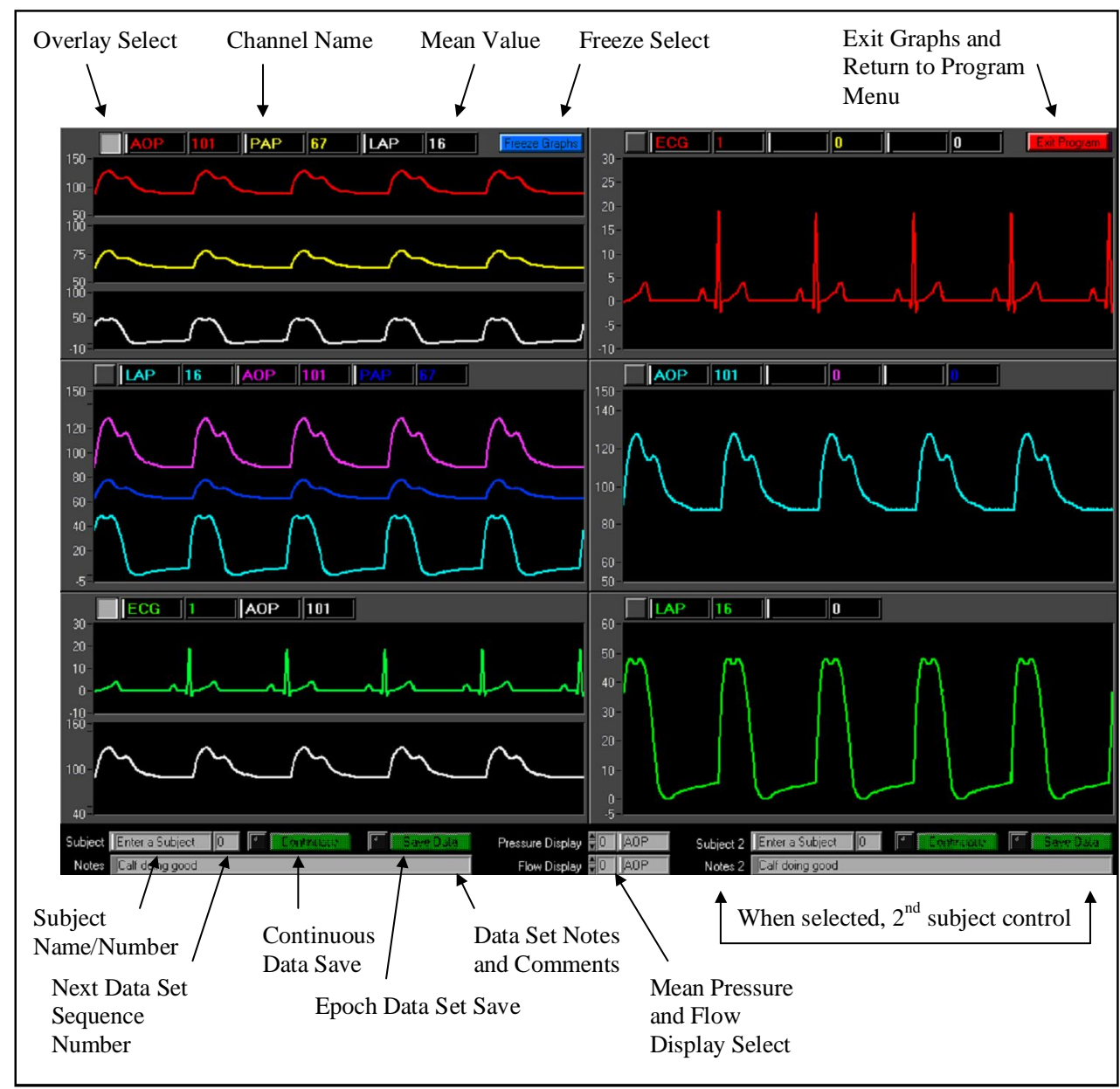

FIGURE 23 - The DAQ system allows for real-time monitoring and collection of data from waveforms produced from pressure and flow transducers (30). 


\section{Adult Circulatory Mock Flow Loop}

In order to study the flow characteristics of the cannula under physiological like conditions, an adult mock circulatory flow loop was utilized. Investigators at the Cardiovascular Innovation Institute have developed the mock loop mainly for testing of cardiac devices in normal and pathologic states (7). Pantalos et al. describe the in vitro circulatory system, which can produce physiologically equivalent pressures, volumes, flows as to faithfully reproduce normal, heart failure, and partial cardiac recovery physiologic states as a platform for testing platform for testing cardiac devices (7).This mock loop circuit was used to complete in vitro dynamic flow testing on the cannula (Figure 24).

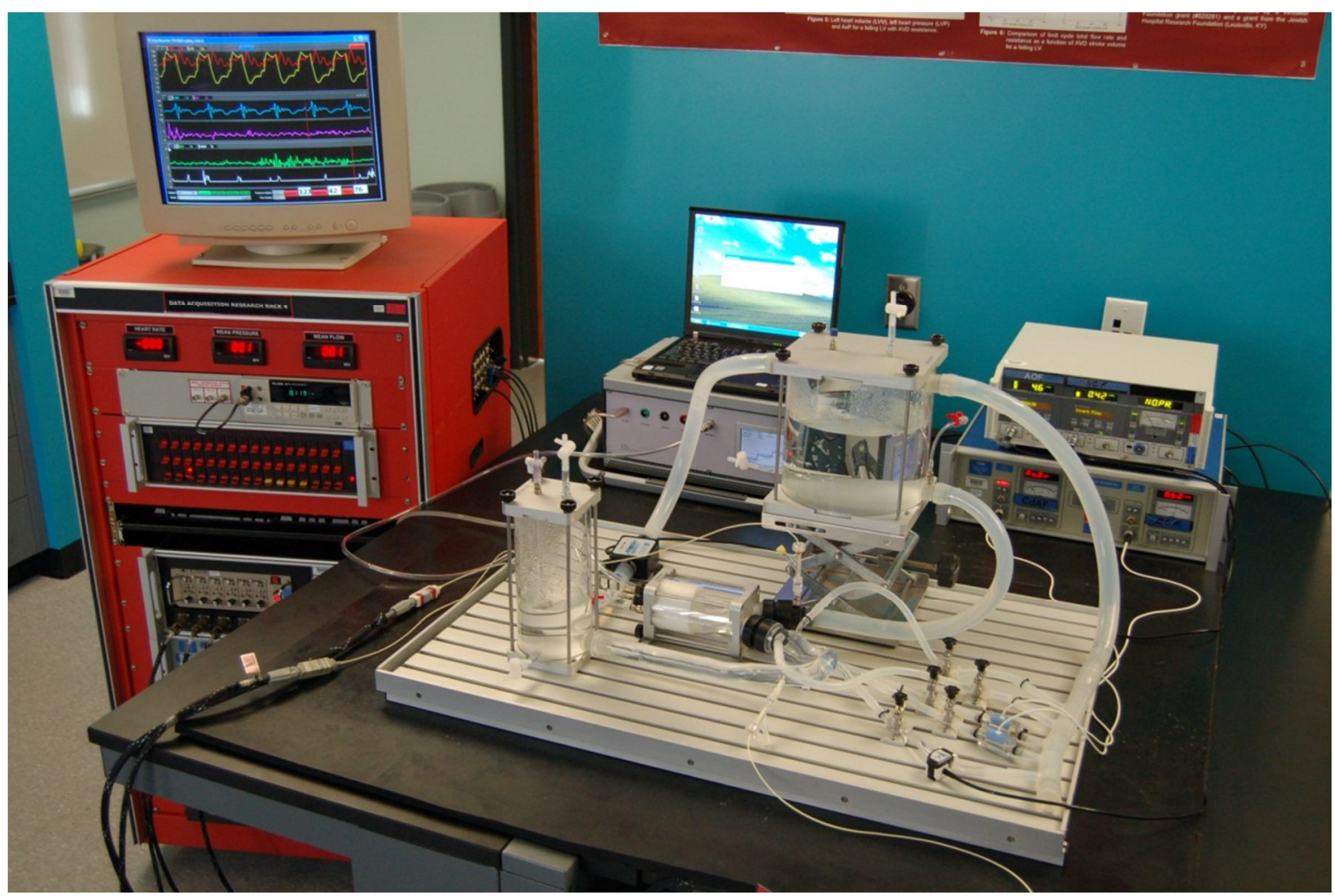

FIGURE 24 - Adult circulation mock flow loop developed by Pantalos et al (7).

Physiologically equivalent pressures, volumes, flows are used to reproduce normal, heart failure, and partial cardiac recovery physiologic states of the heart. 
The adult mock flow loop consists of a mock ventricle with atrial and apical inflow cannulation sites, mock systemic vasculature with aortic root and descending thoracic aorta outflow cannulation sites, and mock coronary vasculature. The mock ventricle is powered and controlled by a pneumatic driver. Figure 25 gives a diagram of the mock loop. This mock loop is able to faithfully produce the Frank-Starling response with physiologic characteristic hemodynamic parameters and pressure-volume relationships under a variety of testing conditions. The cardiac and vascular hemodynamic waveforms of the mock circulation are comparable to natural physiology in both magnitudes and morphologic features.

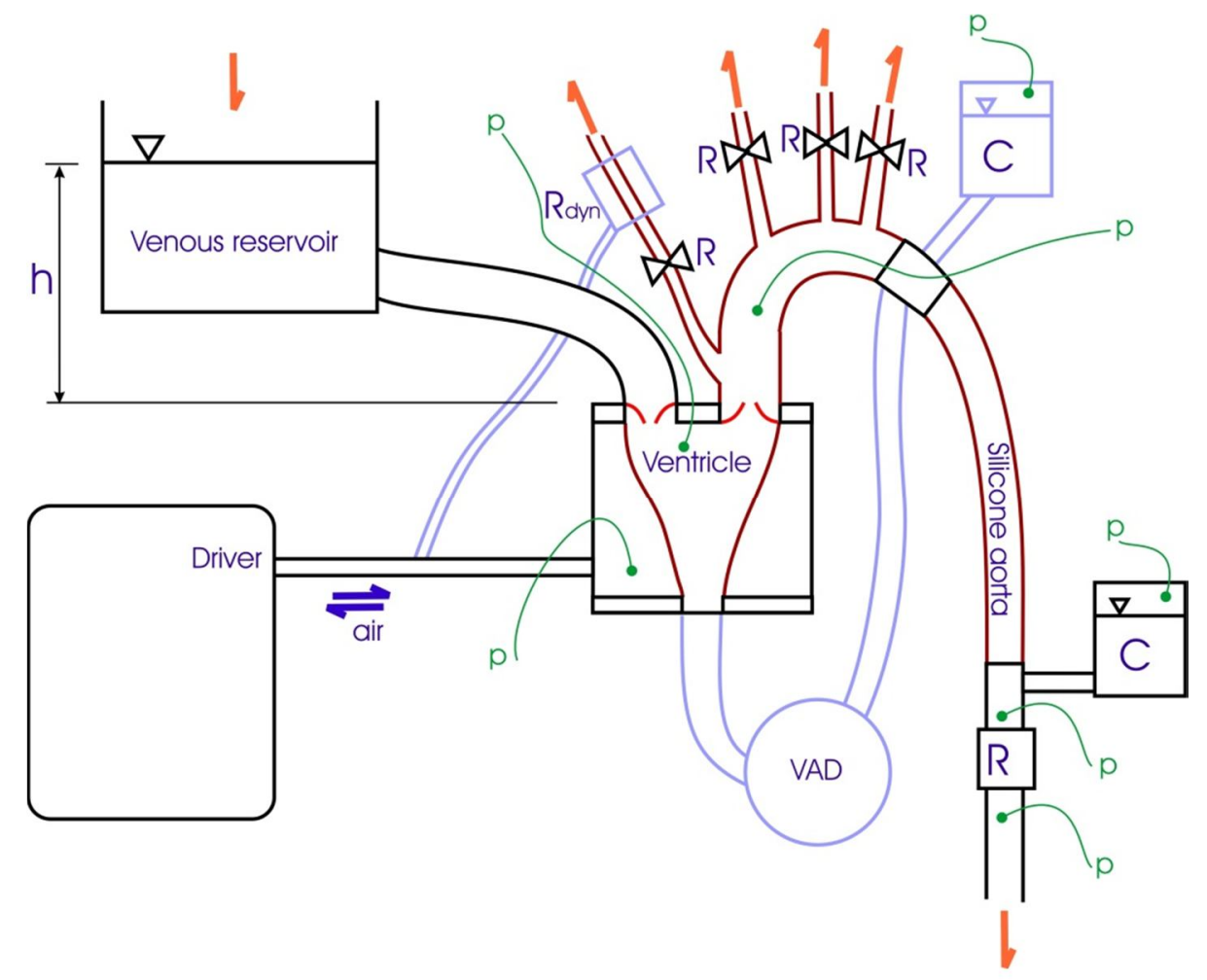

FIGURE 25 - Diagram of the adult circulation mock flow loop developed by Pantalos et al (7). Due to its versatility and robustness, the mock loop is able to test the AVB system providing a powerful in vitro testing environment. 
The versatility of this system allowed the entire aortic valve bypass system to be tested. The cannula was introduced to the apical cannulation site of the mock ventricle and was connected to a conduit and valve eventually connecting to a T-junction in the mock descending aorta. This setup allows for in vitro testing of the AVB system as a whole and provides conditions very similar to what the cannula would experience clinically. Using the pressure and flow instrumentation previously described, the mock loop was instrumented to measure: Aortic Flow (AoF), Aortic Pressure (AoP), Left Ventricular Pressure (LVP), Left Atrial Pressure (LAP), Flow though the AVB conduit (GRF), and Pressure inside the AVB conduit (GRP).

Choice of fluid was also important in creating near physiologic testing conditions. Glycerol was used as a blood analog in the flow loop. The viscosity of the glycerol used in the loop was 3.7 centipoise, which is very similar to blood. Investigators have shown that this material provides a good blood analog fluid for flow conditions characteristic of large arteries. Because the fluid is transparent and made from readily available and fairly inexpensive materials, it may be generally useful for in vitro hemodynamic studies, particularly those employing optical techniques (33).

\section{Computer Software Package}

A host of computer software programs were used in the design and analysis of the cannula. AutoCAD (AutoCAD 2008, San Rafael, CA) was used to create the initial model while SolidWorks (SP3.0 2009, Dassault Systems) was used to create the final cannula and AVB system model. The schematics and CAD data files were sent to ATS Medical for fabrication. Primary analysis of data collected from mock loop experiments 
and CFD computations was completed using the MATLAB (MathWorks, Inc., Natick, MA) software package.

Characterizing hemodynamic parameter values were calculated using the custom MATLAB program HEART (Hemodynamic Evaluation and Assessment Research Tool) developed by Schroeder et al. (34). All hemodynamic parameters were calculated on a beat to beat basis and averaged over the entire data set to obtain a mean value for each parameter. Sample hemodynamic waveforms for each data epoch and test condition were plotted for post-analysis to validate waveform fidelity. Microsoft Office 2007 software package (Excel, Word, PowerPoint) was also used for preparation of graphs and tables. Computation flow analysis was completed with the ANSYS CFD (ANSYS Inc., Canonsburg, PA, U.S.A.) software package. The CAD files of the cannula designs were loaded into ANSYS workbench project and the 3D models were checked for continuity. The model was then meshed using the AutoMesh function with the appropriate settings for fluid flow. Pre-solver software was used to define boundary regions and initial conditions. The CFX solver was then utilized and the computation ran until the solving equations converged upon a final solution. Post-solver component was then used to analyze and visualize the results. Stream lines, gradient profiles, vector fields, and slice planes were used for visual analysis while average, peak, and minimum values for various parameters such as flow rate, pressure, shear rate, and velocity at the boundary regions were calculated. 


\section{RESULTS}

\section{$\underline{\text { Static Flow Loop }}$}

The data was then plotted with the pressure drop vs. flow rate to determine the flow characteristics. As discussed previously, the resistance of the cannula can be measured by taking the change in pressure and dividing by the flow rate.

$$
\mathrm{R}=\Delta \mathrm{P} / \mathrm{Q}
$$

The steeper the slope of the pressure drop versus flow rate curve, the higher the overall resistance is to flow. Figure 26 shows the flow profiles for all cannula tested. The resistance decreases significantly as the size of the outer diameter is increased.

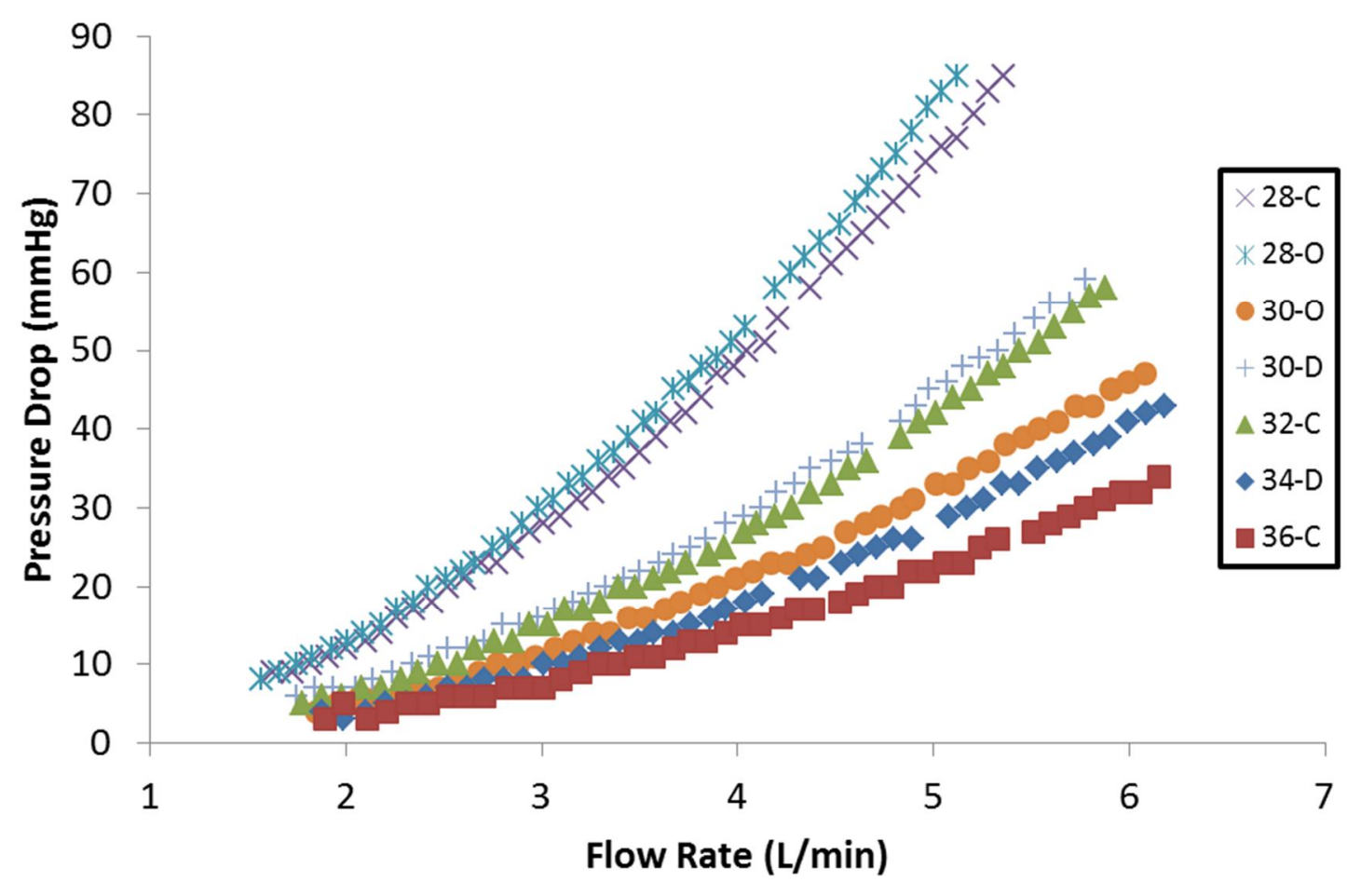

FIGURE 26 - Flow profiles for all seven cannula tested in the static flow loop and shows resistance to flow decreases with increasing diameter size. Legend Key: $\mathrm{C}$ - cage, $\mathrm{O}$ open, D - dome. Outer French (Fr) diameter sizes are given. 
Resistance values and the R-Squared value for each cannula are reported in Table $\mathrm{X}$. The 28Fr (9.3mm diameter) cannulas had the highest resistance values among all the cannula. The $30 \mathrm{Fr}$ cannula had almost half the resistance of the $28 \mathrm{Fr}$ cannulas and resistance decreases slightly as size is increased from this size. The R-squared values are high with all cannula greater than 0.979 showing good linear approximation fit.

TABLE X

STATIC LOOP RESULTS FOR TESTED CANNULAS

\begin{tabular}{|c|c|c|}
\cline { 2 - 3 } \multicolumn{1}{c|}{} & $\begin{array}{c}\text { Resistance } \\
\text { (linear regression } \\
\text { model) }\end{array}$ & R-Squared \\
\hline $28-C$ & 20.9 & 0.984 \\
\hline $28-0$ & 21.9 & 0.982 \\
\hline $30-D$ & 13.3 & 0.981 \\
\hline $30-0$ & 10.4 & 0.982 \\
\hline $32-C$ & 13.1 & 0.979 \\
\hline $34-D$ & 9.4 & 0.979 \\
\hline $36-C$ & 7.3 & 0.979 \\
\hline
\end{tabular}

Legend: C - cage, $\mathrm{O}$ - open, D - dome. Outer French (Fr) diameter sizes are given. Units of Resistance $=\left(\mathrm{mmHg}^{*} \mathrm{~min}\right) / \mathrm{L}$

\section{Blood Trauma}

Table XI presents the results from the blood chemistry and plasma free hemoglobin testing. Over the course of the 6 hour study all the blood chemistry stayed within normal values for the bovine model. The WBC, RBC, HGB, HCT, and PLT counts all decreased slightly while the plasma free hemoglobin slightly increases over the study period though this can be expected as the blood outside the body degrades over time. 
All plasma free hemoglobin measurements were well under $100 \mathrm{mg} / \mathrm{dL}$ indicating little to no hemolysis. The device, the HemoCue Plasma/Low Hb, used to measure the plasma free hemoglobin has a linear measuring range between 0.3-30.0 g/L. The device manufacturer advises caution for evaluating instrument readings below 30mg/dL.

\section{TABLE XI}

\section{RESULTS FROM TRAUMA MOCK LOOP TESTING}

\begin{tabular}{|c|c|c|c|c|c|c|c|c|c|c|c|}
\hline Time & Condition & $\begin{array}{c}\text { Blood wt. } \\
\text { g }\end{array}$ & $\begin{array}{l}\text { Flow } \\
\text { Ipm }\end{array}$ & $\begin{array}{c}\text { Pressure Drop } \\
\mathrm{mmHg}\end{array}$ & $\begin{array}{l}\text { ACT } \\
\text { sec }\end{array}$ & $\begin{array}{c}\text { WBC } \\
\text { thous/uL }\end{array}$ & $\begin{array}{c}\mathrm{RBC} \\
\mathrm{mill} / \mathrm{uL}\end{array}$ & $\begin{array}{c}\text { HGB } \\
\%\end{array}$ & $\begin{array}{c}\mathrm{HCT} \\
\%\end{array}$ & $\begin{array}{c}\text { PLT } \\
\text { thous/ul }\end{array}$ & $\begin{array}{l}\mathrm{pfHb} \\
\mathrm{mg} / \mathrm{dL}\end{array}$ \\
\hline 1030 & Baseline & 651 & 2.4 & 40 & $>300$ & 5.34 & 6.8 & 8.6 & 22.7 & 889 & 10 \\
\hline 1200 & Sample & -10 & 2.4 & 40 & $>300$ & 5.46 & 6.77 & 8.6 & 22.5 & 877 & 20 \\
\hline 1330 & Sample & -10 & 2.4 & 42 & $>300$ & 5.39 & 6.77 & 8.6 & 22.5 & 863 & 20 \\
\hline 1500 & Sample & -10 & 2.4 & 41 & $>300$ & 5.3 & 6.77 & 8.6 & 22.5 & 851 & 30 \\
\hline 1625 & Sample & -10 & 2.4 & 40 & $>300$ & 4.93 & 6.72 & 8.6 & 22.4 & 846 & 35 \\
\hline 1635 & Sample & -5 & 2.4 & 40 & $>300$ & 4.9 & 6.7 & 8.6 & 22.4 & 824 & 40 \\
\hline
\end{tabular}

The $\mathrm{pfHb}$ increased over the experiment to a final reading of $40 \mathrm{mg} / \mathrm{dL}$, which is slightly larger than the minimal detection levels of the HemoCue again showing no significant levels of hemolysis.

\section{Dynamic Flow Loop}

The flow rates for each cannula are presented in Table XII. For severe AS, the peak flow rate through the preliminary prototype was $0.6 \mathrm{~L} / \mathrm{min}$, while the $32 \mathrm{Fr}$ and $36 \mathrm{Fr}$ provided flow rates of $1.65 \mathrm{~L} / \mathrm{min}$ and $2.55 \mathrm{~L} / \mathrm{min}$, respectively. With the aortic root fully clamped off, the maximum flow rates through the prototype cannula was $1.49 \mathrm{~L} / \mathrm{min}$, while the $32 \mathrm{Fr}$ and $36 \mathrm{Fr}$ had flow rates of $3.31 \mathrm{~L} / \mathrm{min}$ and $3.87 \mathrm{~L} / \mathrm{min}$, respectively. 
TABLE XII

PEAK FLOW RATES FOR CANNULA UNDER DYNAMIC TESTING CONDITIONS

\begin{tabular}{lccc} 
& \multicolumn{3}{c}{ Flow (L/ min) } \\
\cline { 2 - 4 } Aortic Stenosis & Prototype & $32 \mathrm{Fr}$ & $36 \mathrm{FR}$ \\
\hline None & 0.34 & 0.85 & 1.17 \\
Mild & 0.40 & - & 1.15 \\
Moderate & 0.57 & 1.4 & 1.7 \\
Severe & 0.6 & 1.65 & 2.55 \\
Complete & 1.49 & 3.31 & 3.87 \\
\hline
\end{tabular}

The AVB system showed a trend of decreased transvalvular pressure difference for all three cannula under most conditions. Figure 27 shows the baseline valve gradient with the AVB arm clamped off and the valve gradient when the AVB system is in use. The largest difference can be seen for the severe arotic stenosis condition although the preliminary prototype showed no improvement. 


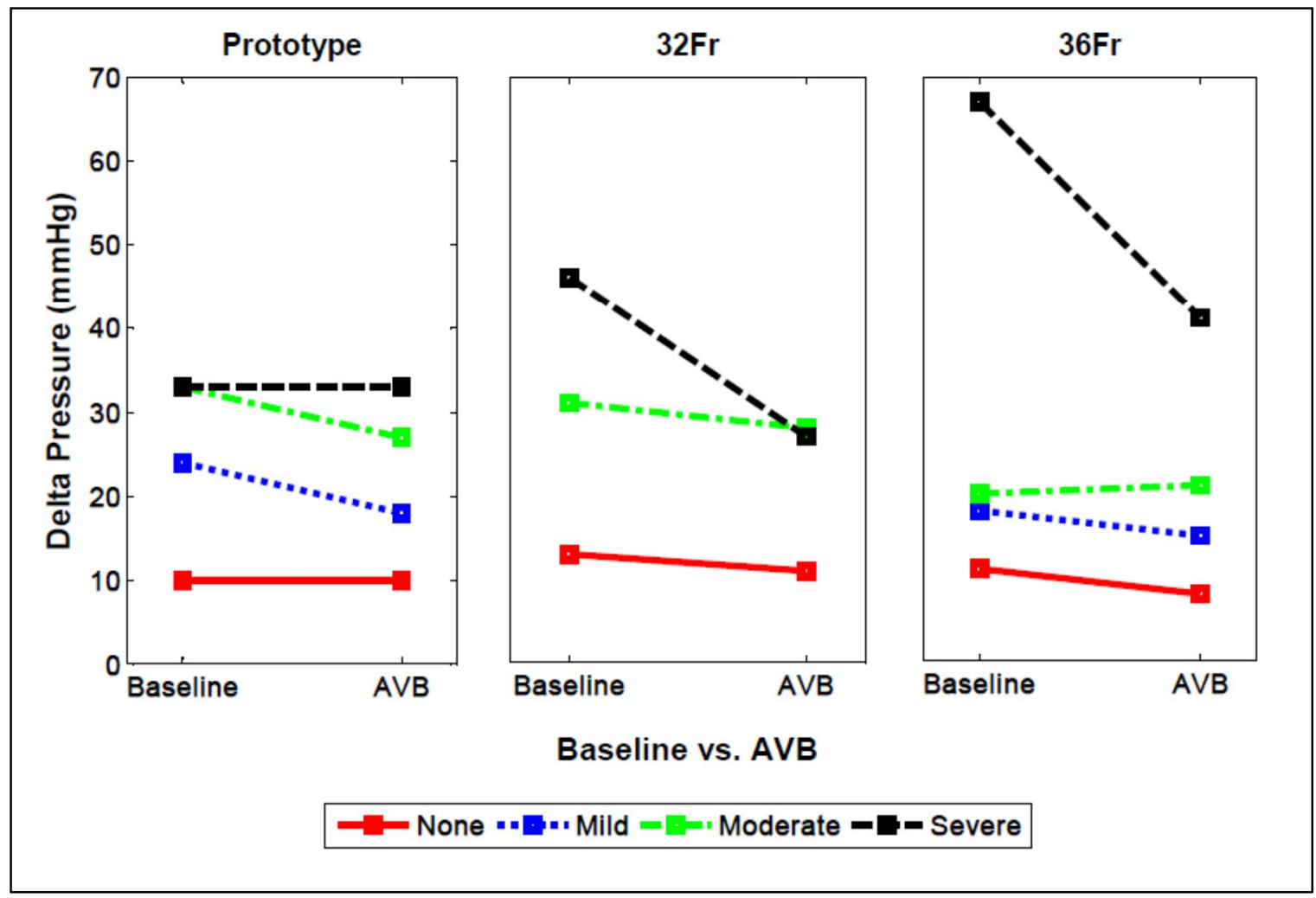

FIGURE 27 - Difference in valve gradient (LVP - AoP) from the adult circulation mock flow loop between baseline and with the AVB system. Improvement can be seen as the cannula diameter increases. The largest improvement can be seen in the $36 \mathrm{Fr}$ cannula for the severe aortic stenosis condition.

Figure 28 shows the $\%$ conduit flow increases with cannula size. The prototype again showed poor performance, as only $15 \%$ of the fluid flow was through the cannula. The $32 \mathrm{Fr}$ cannula showed an improvement to $36 \%$, while the $36 \mathrm{Fr}$ cannula had the best performance at $59 \%$. 


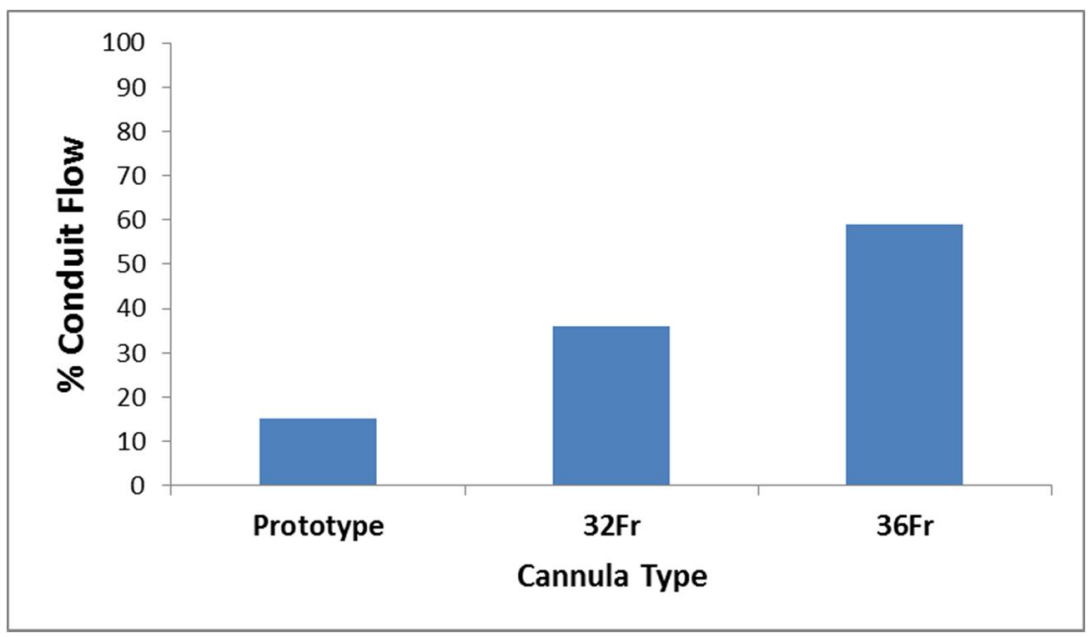

FIGURE 28 - Relative conduit flow as a percentage of total cardiac output. \%

Conduit flow provides a measure of the effectiveness of the cannula in the AVB system. The percentage increased as cannula size was increased.

\section{Computational Dynamic Flow Analysis}

Two studies were performed on a $12 \mathrm{~mm}$ diameter $(36 \mathrm{Fr})$ cannula to see the effect of number and size of the sideholes on wall shear stress, shear strain rate, and flow characteristics. For each computational run, a custom report was used to gather the flow parameters and create figures. A sample report is presented in Appendix IV.

\section{Number of Sideholes Study}

The flow rates at the outlet for each cannula are presented in Figure 29. Flow slightly increased as the number of sideholes increased, although, the cannula with no sideholes had the highest flow of $4.1 \mathrm{~L} / \mathrm{min}$. These values remain very similar to one another with the largest difference being $0.3 \mathrm{~L} / \mathrm{min}$. 


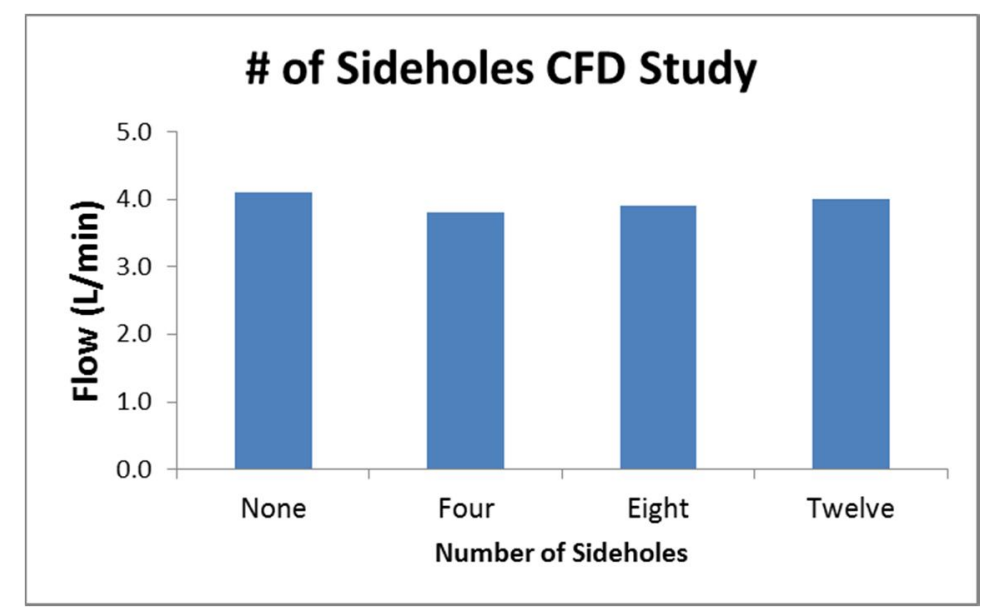

FIGURE 29 - Flow rates for $12 \mathrm{~mm}$ diameter (36Fr) cannula with different numbers of sideholes.

Figure 30 presents a cross sectional view of the velocity profiles for all four cases. The largest velocities are seen in the cannula with no sideholes. In the cannulas with sideholes, the max velocities occur at the bottom of the most distal sideholes. These high velocity areas are the same areas that contained the largest shear stress, seen in Figure 31. 


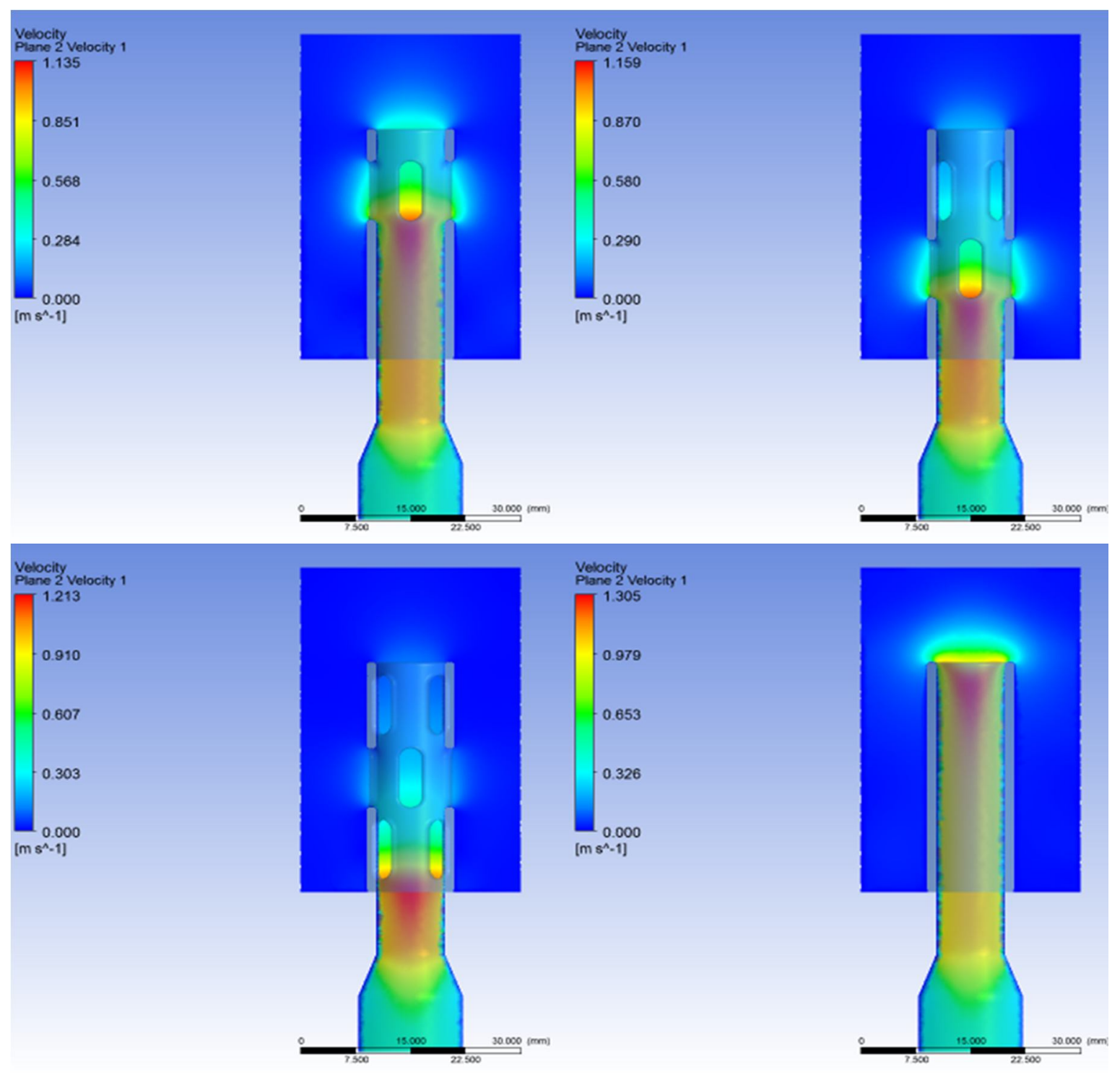

FIGURE 30 - Velocity profiles for the number of sideholes study. Four (Top Left), Eight

(Top Right), Twelve (Bottom Left), and No Sideholes (Bottom Right). 


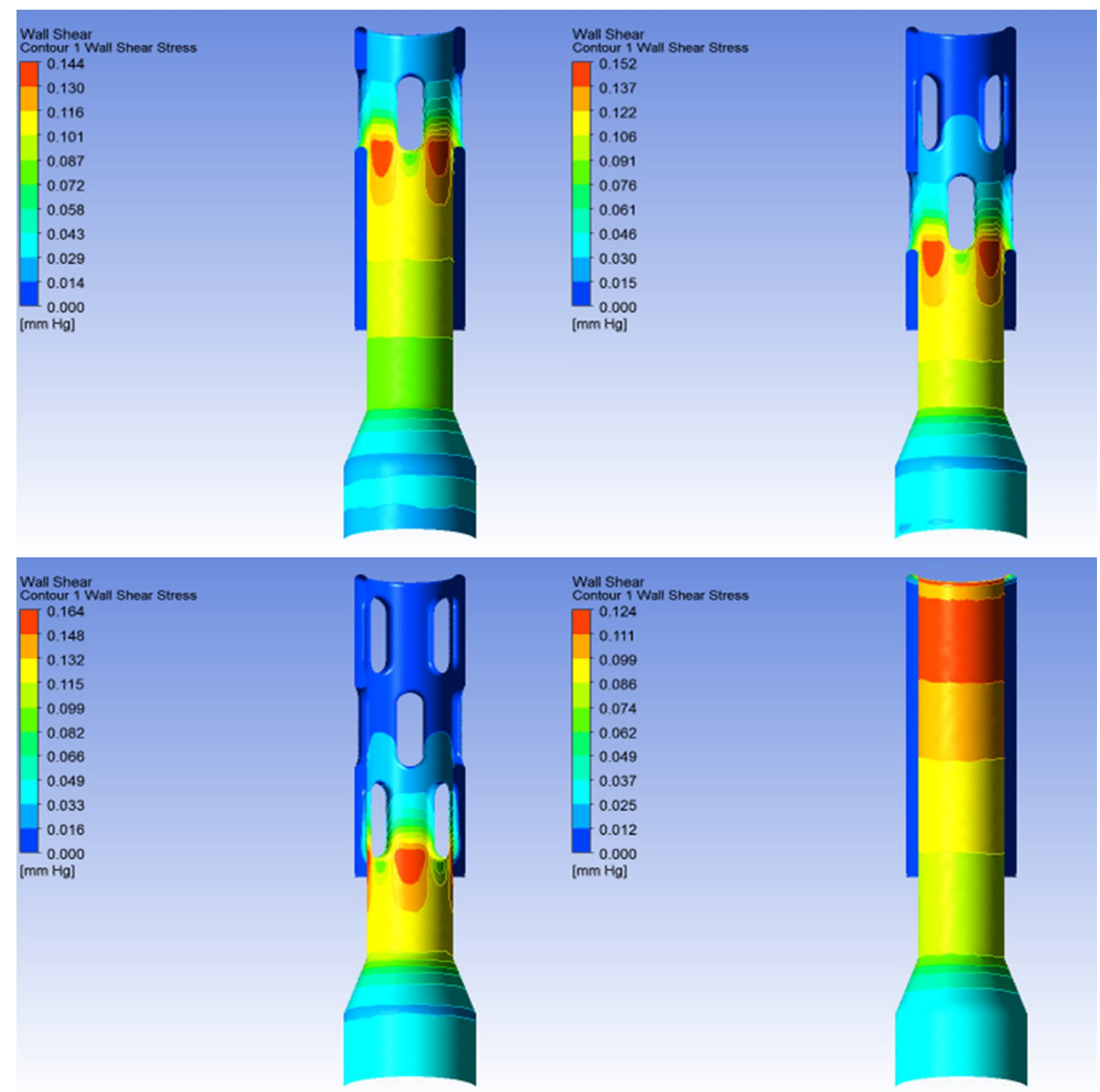

FIGURE 31 - Shear wall stresses for the number of sideholes study. Four (Top Left),

Eight (Top Right), Twelve (Bottom Left), and No Sideholes (Bottom Right). The highest wall shear rates are seen on the bottom edges of the most distal sideholes.

Flow rate and Reynolds numbers at the outlet as well as shear wall stress and strain rate are reported in Table XIII. The reported Reynolds numbers for all cases were greater than 3000 indicating turbulent flow at the outlet. Shear strain rate decreased as sideholes are increased. Flow and maximum strain rate both increase with addition of 
sideholes though the No sideholes cannula has the largest values. Maximum shear stresses increases with addition of sideholes.

TABLE XIII

RESULTS OF NUMBER OF SIDEHOLES STUDY

\begin{tabular}{lcccc} 
& \multicolumn{4}{c}{ Number of Sideholes } \\
& None & Four & Eight & Twelve \\
\hline Flow (L/ $\mathbf{m i n})$ & 4.1 & 3.8 & 3.9 & 4.0 \\
Reynolds \# & 3349 & 3096 & 3174 & 3293 \\
$\begin{array}{l}\text { Wall Shear Stress (mmHg) } \\
\text { Max Shear Stress }\end{array}$ & 0.124 & 0.144 & 0.152 & 0.165 \\
$\begin{array}{l}\text { Shear Strain Rate (s^-1) } \\
\text { Max Shear Rate }\end{array}$ & $\begin{array}{c}258.4 \\
891\end{array}$ & $\begin{array}{c}187.6 \\
851\end{array}$ & $\begin{array}{c}141.1 \\
829\end{array}$ & $\begin{array}{c}118.4 \\
876\end{array}$ \\
\hline
\end{tabular}

Size of Sideholes Study

The flow rates at the outlet for each of the four cannulas are presented in Figure 32. Flow rate increased as size increased. Similar to the other study, the largest differences in flow between the cannulas was only $0.3 \mathrm{~L} / \mathrm{min}$. The cannula with no fillet had slightly more flow than the medium sideholes but remained less than the large sideholes. 


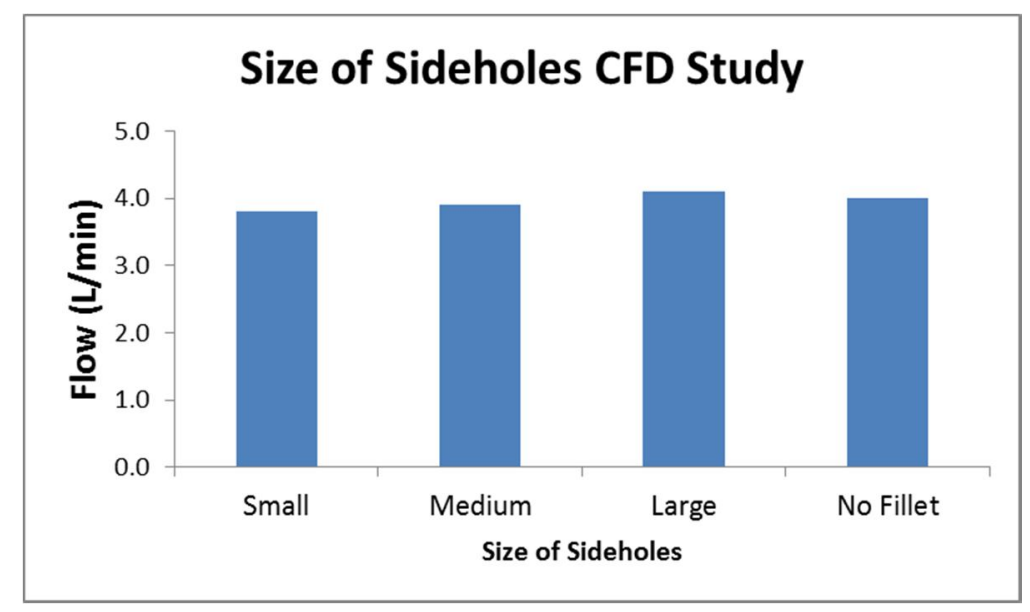

FIGURE 32 - Flow rates for cannula in the size of sideholes study. The cannula with no fillet has the highest flow rates.

The cross sectional velocity profiles are presented in Figure 33. Similar to the number of sideholes study, the highest velocities are seen on the bottom edges of the sideholes most distal from the tip. The cannula with small sideholes experienced the largest wall shear stresses, which decrease with increasing size shown in Figure 34. 


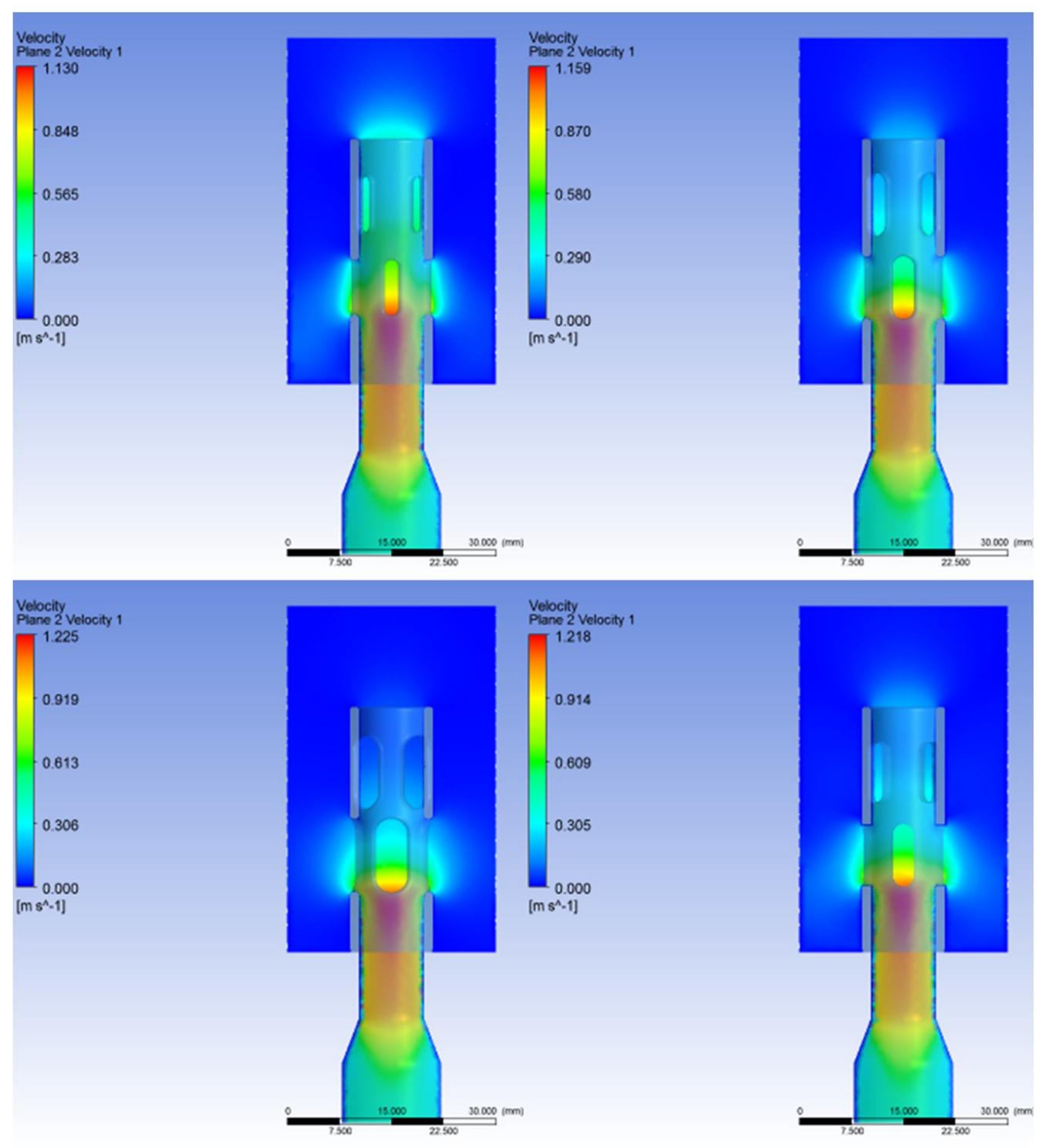

FIGURE 33 - Velocity profiles for the size of sideholes study. Small (Top Left),

Medium (Top Right), Large (Bottom Left), No Fillet (Bottom Right). 


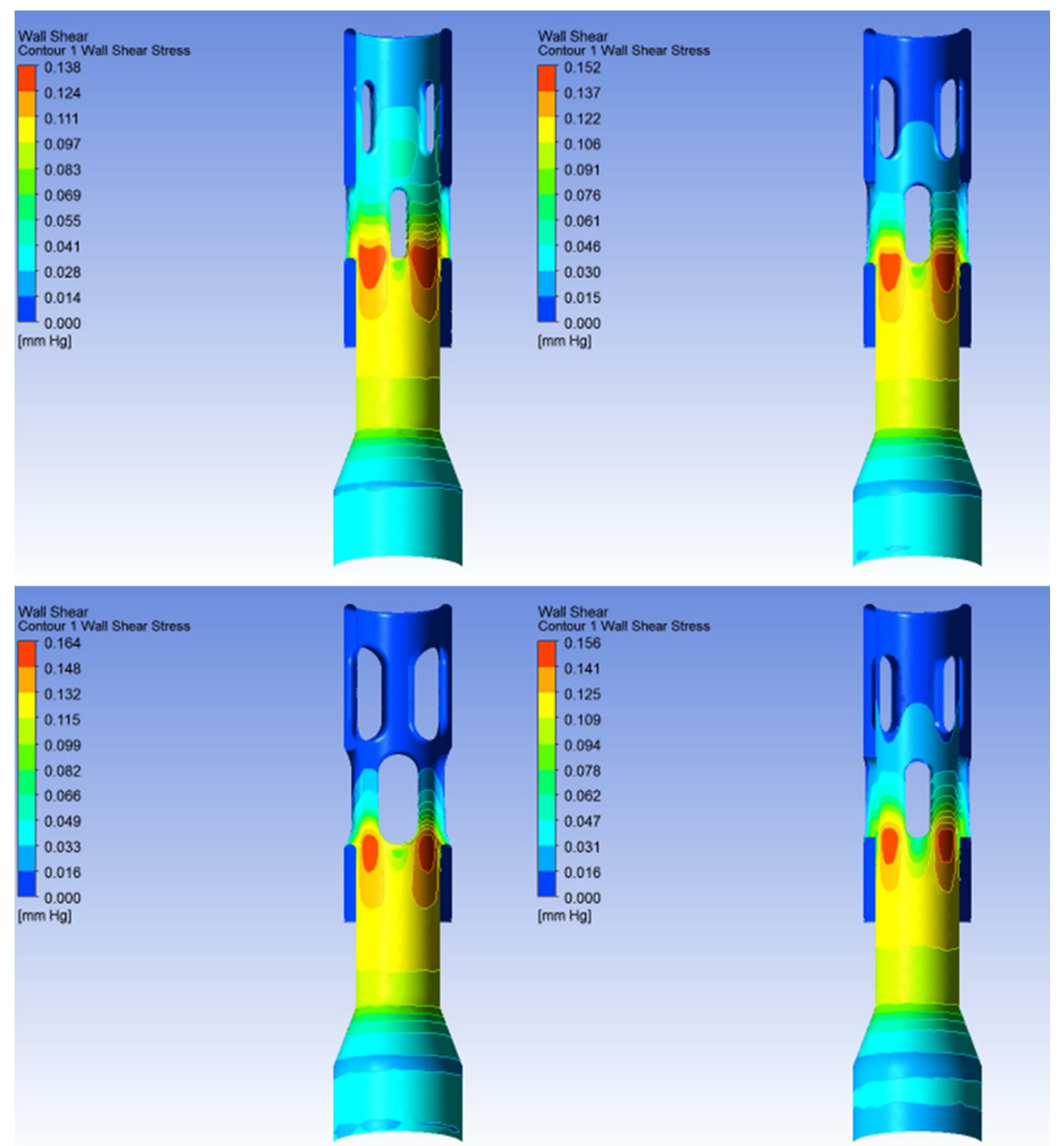

FIGURE 34 - Shear wall stresses for the size of sidehole study. Small (Top Left), Medium (Top Right), Large (Bottom Left), No Fillet (Bottom Right)

The results of this experiment are presented in Table XIV. Similar to the number of sideholes study, all cases have turbulent flow. Flow rate, Reynolds number and maximum shear stress increased with increasing size. The No fillet cannula showed slightly higher flow, less shear stress and less strain rate than the medium cannula. 
TABLE XIV

RESULTS OF SIZE OF SIDEHOLES STUDY

Size of Sideholes

\begin{tabular}{lcccc} 
& Small & Medium & Large & No Fillet \\
\hline Flow (L/min) & 3.8 & 3.9 & 4.1 & 4.0 \\
Reynolds \# & 3093 & 3174 & 3320 & 3227 \\
$\begin{array}{l}\text { Wall Shear Stress (mmHg) } \\
\text { Max Shear Stress }\end{array}$ & 0.1383 & 0.152 & 0.164 & 0.156 \\
$\begin{array}{l}\text { Shear Strain Rate (s^-1) } \\
\quad \text { Max Shear Rate }\end{array}$ & 189.5 & 141.1 & 109.3 & 68.8 \\
\hline
\end{tabular}

\section{DISCUSSION}

\section{Static Loop}

The results from this experiment provide two insights. First that the resistance to flow decreases with increasing cannula diameter and second that an "open" tip design, which is a design that incorporates an orifice at the most distal tip featuring 8 sideholes arranged in 2 sets of 4 openings, seen in Figure 35, is preferred over other designs studied. The diameter plays a large role in the resistance while the tip design plays a much smaller but still important role. These results were used to aid the design characteristics chosen for the final AVB model. 


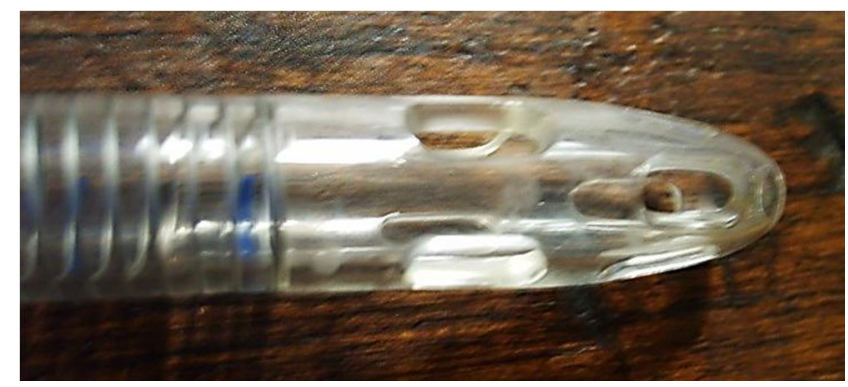

FIGURE 35 - 'Open’ tip design that includes an open orifice with 8 sideholes displayed the best flow characteristics from the three designs studied in static loop testing.

This data shows the significant differences between the different sized cannula. The resistance values for the $28 \mathrm{Fr}$ cannulas are as high as 21.9 while the $36 \mathrm{Fr}$ cannula remains in single digits at 7.3. The R-Square values that represent the linear approximation of the resistances are correlated well and show a good fit. It is evident from the data that there is a sharp difference between the $28 \mathrm{~F}$ and $30 \mathrm{Fr}$ compared to the other increments. This suggests that the diameter plays a large role in the resistance and at a critical point between these two sizes. It may be undesirable to have an apical cannula this small due to the likely drop in flow rates. From this data it is favorable to select a cannula size of $30 \mathrm{Fr}(10 \mathrm{~mm})$ or larger.

To validate the data obtained, pressure drop vs. flow rate graphs were compared to ones obtained from the cannula manufacturer (35). The data obtained from the static loop testing closely matches the pressure/flow plots obtained from the manufacturer, an example can be seen in Figure 36. The figure shows that the flow rate and pressure drop values measured (Figure 36- right) are almost identical to the values obtained from the manufacturer (Figure 36- left). 

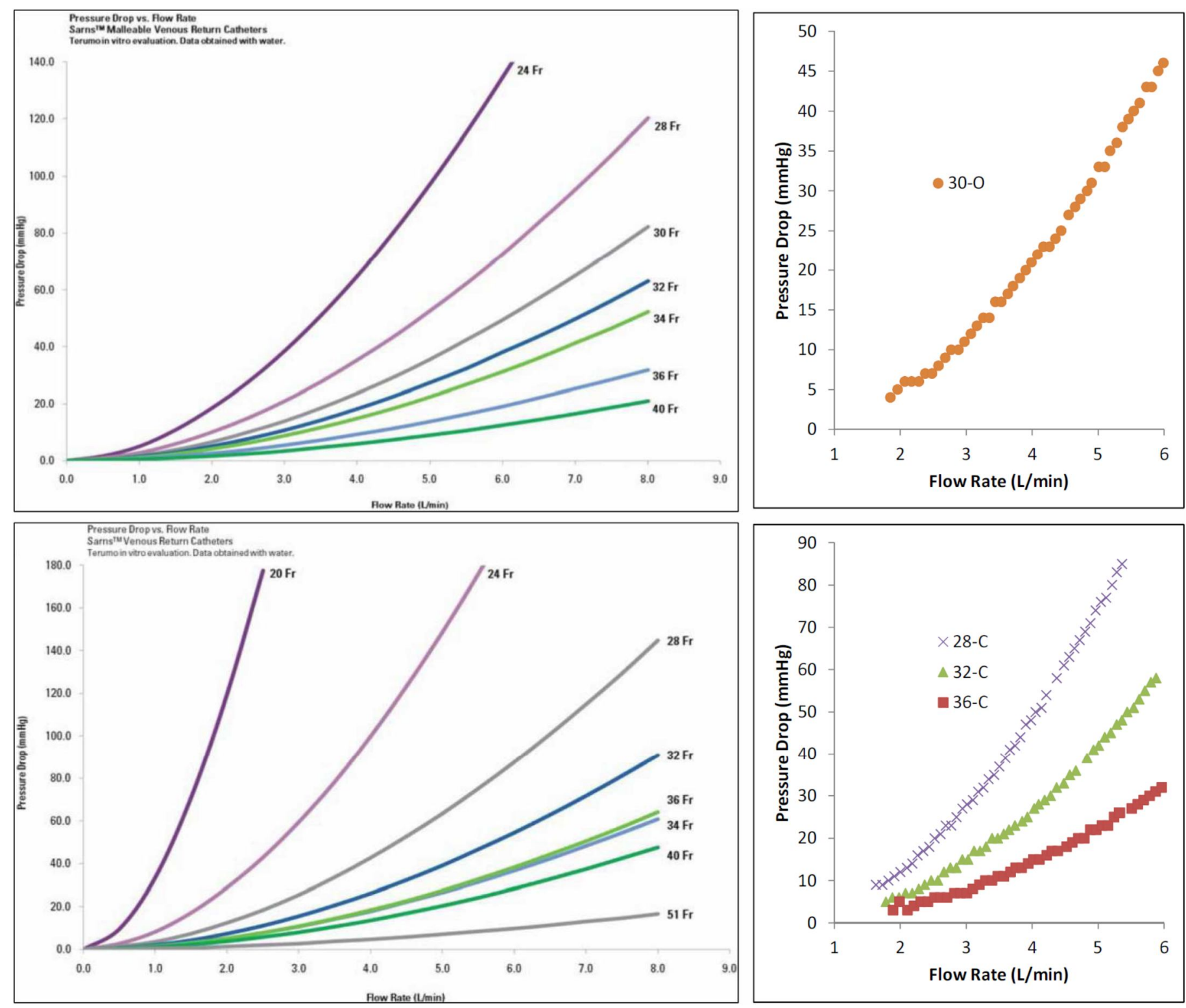

FIGURE 36 - Pressure Drop vs. Flow Rate data obtained from the cannula manufacturer to the left with data obtained from static loop testing on the right. The top figure shows the ‘open’ tip Sarns Malleable Venous Return Catheters while the bottom shows the 'cage' tip Sarns Venous Return Catheters. The data obtained from the manufacturer closely matched data obtained and validates observations made in the experiment. 
This data from the manufacturer also shows the large differences seen as the diameter of the cannula is decreased. Specifically, this data also shows that the $28 \mathrm{Fr}$ and smaller cannula show a significantly increased resistance compared to larger cannula. Also the critical point where the resistance increases dramatically is in the area between 28Fr and 32Fr for all three of the cannula designs. This confirms the observations that the diameter size plays the most important factor in resistance to flow and the design of the tip plays a secondary but important role.

Comparing the plots between the three designs, the results show that the open tip design has lower flow resistance across almost all sizes. It can be inferred that the orifice at the tip with the addition of sideholes along the body are important features that contribute to improved flow characteristics.

Explanation of these results can be found in comparison of the features of the three designs. The cage design is primarily a design where the tip allows flow through openings at the very distal end and features no sideholes along the body of the tip. The dome design, in contrast, has no opening at the distal most point and has all of the flow come through 8 sideholes along the body of the tip. It seems that the open design features both of these characteristics with an orifice at the distal tip and sideholes along the body and show superior flow characteristics compared to the dome and cage designs.

\section{Blood Trauma}

The results from this experiment indicate that there is minimal to no hemolysis caused by flow in the preliminary prototype. This study gives confidence for use of the 
fabrication material (pyrolytic carbon) and the tip design of the cannula (one with an open orifice at the distal most tip with sideholes along the body of the tip).

The results from this study show minimal to little hemolysis using the preliminary prototype cannula. The cannula has an outer diameter of $7 \mathrm{~mm}$ at the distal-most tip and as such had a higher resistance to flow compared to the cannulas studied in all experiments performed. The values for all blood chemistry and plasma free hemoglobin showed little change from baseline to the study endpoint. The size and design of the cannula did not shear or damage the red blood cells in a significant manner.

The biocompatibility of the pyrolytic carbon was shown to be a good choice as a fabrication material. The strong track record for use of this material in other medical devices, most notably mechanical valves, has been well documented and is discussed previously. The use of the anticoagulation drug, heparin, prevents an analysis on possible thrombus formation, though the use of CFD can pinpoint possible stagnation points.

\section{Dynamic Flow Loop}

The results from the adult mock circulation testing are similar to those obtained in the static loop testing. The results show that the preliminary prototype is too small to be clinically effective as a treatment for aortic stenosis. Flow rate and \% Total flow through the conduit increased with increasing cannula size. The results show a trend of improvement in valve pressure difference between stenotic baseline conditions and the mock loop circuit with the AVB system. These results aid in development of a final design for the AVB system. 
These results show that the size of the cannula play a critical role in improvement of aortic stenosis condition. This data also shows substantial improvement only when the aortic stenosis is severe. There is a significant difference between the prototype and 32Fr compared to the difference between the $32 \mathrm{Fr}$ and $36 \mathrm{Fr}$. These results are similar to the static flow results where there exists a critcal point between $30 \mathrm{Fr}$ and $32 \mathrm{Fr}(10 \mathrm{~mm}$ and $10.7 \mathrm{~mm}$ ) in the tip size that critically impedes flow through the cannula.

When studying the $\%$ conduit flow, the $36 \mathrm{Fr}$ shows improved performance over the 32Fr and prototype. For comparison, a recent investigator presenting early and midterm hemodynamics after AVB surgery reported initial relative $\%$ conduit flow to be $63 \%+-10 \%$ for 47 patients who had received AVB surgery (29). These results show that the $36 \mathrm{Fr}$ cannula is the best choice for treatment of severe aortic stenosis of the three cannulas studied. The size of the $36 \mathrm{Fr}$ cannula $(12 \mathrm{~mm})$ may be the smallest cannula tip size that will still give significant improvement in the valve gradient for patients with aortic stenosis.

\section{Computational Flow Dynamics Analysis}

The two blood damage phenomena potentially occurring in an in implant of a blood contacting artificial material are thrombosis and hemolysis. Thrombosis refers to the formation and growth of blood clots. Hemolysis is hypothesized to be a function of cellular exposure to high shear stress and the length of time over which this exposure occurs. The processes that cause thrombosis and hemolysis to occur in complex flow situations are still not completely understood. The general understanding is that cellular exposure to high shear stresses can lead to hemolysis (36) and platelet activation (37) 
while regions of low shear stress and/or flow stagnation can be susceptible to thrombus deposition (38).

Blood is a suspension consisting of primarily red blood cells, along with other formed elements which contribute to its viscoelastic behavior. Blood behaves as a Newtonian fluid at shear rates above $500 \mathrm{~s}^{-1}$. At shear rates lower than $50 \mathrm{~s}^{-1}$ the viscosity of blood increases exponentially, due to the formation of large aggregates of erythrocytes. The presence of shear and strain stresses are important for the ideal biocompatibility with the body. The best situation is to have shear stress throughout the flow just high enough to prevent aggregation and thrombus growth, but not so high as to cause trauma or platelet activation.

Previous studies done by investigators have found that shear stresses on the order of 1500-4000 dynes/cm2 can cause hemolysis (39). Sub-lethal damage to red blood cells can occur at turbulent shear stresses of 500 dynes/cm2 (40). Platelets are more sensitive and can be damaged by shear stresses ranging from 100-500 dynes/cm 2 though this is also very much dependent on the duration of the applied shear stress (41). The wall shear stress from the CFD analysis can provide a prediction for hemolysis and platelet activation. Comparisons between the cannulas can be made to evaluate design choices.

\section{Evaluation of Cannulas}

The highest flow rates were seen in the No sideholes cannula and the Large sideholes cannula with both having $4.1 \mathrm{~L} / \mathrm{min}$ flow. No sideholes cannula had a lower max shear stress $(0.124 \mathrm{mmHg})$ than the Large cannula $(0.164 \mathrm{mmHg})$. The Large cannula has larger areas of lower shear stresses located near the tip. Although it seems a 
little counterintuitive, the cannula with no sideholes showed the best blood biocompatibility of all the cannulas studied. This does not make this cannula the ideal choice for this application though as the differences between the cannulas is still only slight and other factors such as device safety play a role in selection of the best cannula.

The more fully developed flow in the tube with no sideholes has walls that are washed and the shear stress is safely low giving it better blood compatibility the other cannulas studied. The sideholes, on the other hand, produce high shear stresses at the distal end of their openings, and could cause recirculation regions downstream of the holes that promote thrombus growth and attachment. The increase in number of sideholes in essence makes the tube shorter, since a majority of the flow is coming in through the distal holes and very little is coming through the proximal holes.

The shape of the ventricle around the cannula once inserted likely has a great amount of influence on the direction of flow. The surgical placement of the cannula is paramount to the performance of the cannula. If the cannula was improperly placed with one side of the tip lying against a ventricular wall, then full flow will not be achieved. In terms of safety, the addition of sideholes allows fluid to enter from all directions so flow rates can be more consistent while the ventricle is pumping. As the No sidehole cannula has only one inlet, if something were to occur to block or partially occlude the opening (i.e. cannula is against the ventricular wall) the flow could diminish in the conduit and may allow stagnation of blood in the entire AVB system. 


\section{Wall Shear Stress}

When looking at the wall shear stress, the average may not be a very useful indicator and can be very misleading.. There is so much surface area at the proximal part of the tube that has very low wall shear stress that when added to the higher shear stresses at the distal sideholes, the average shear stresses become diluted. Instead investigation of max shear stress may be more helpful and the areas that they occur.

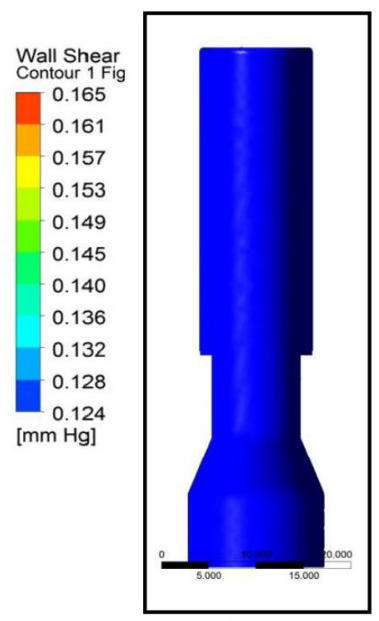

No Sideholes

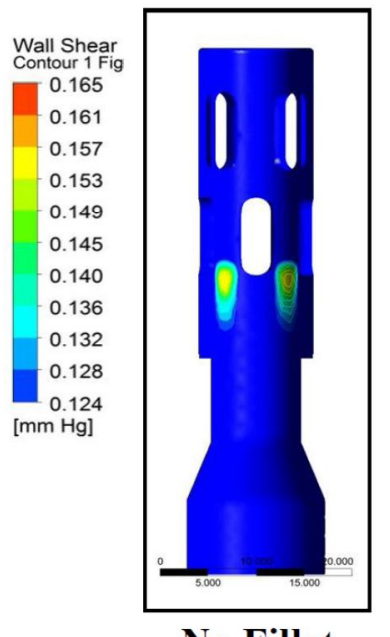

No Fillet

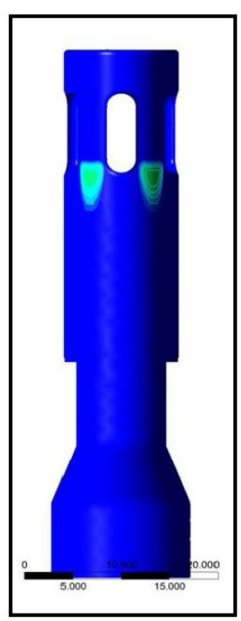

Four

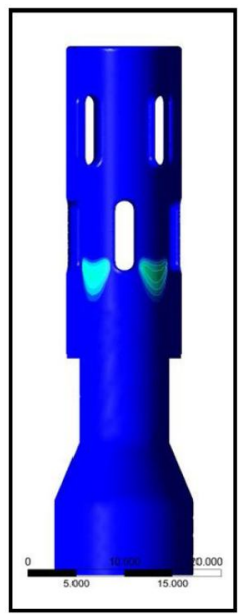

Small

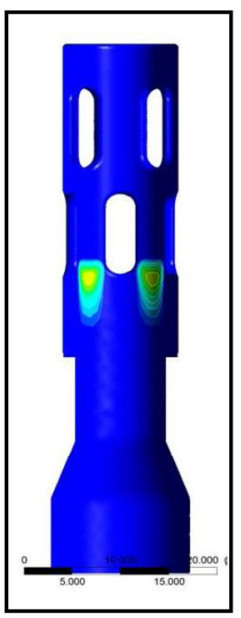

Eight

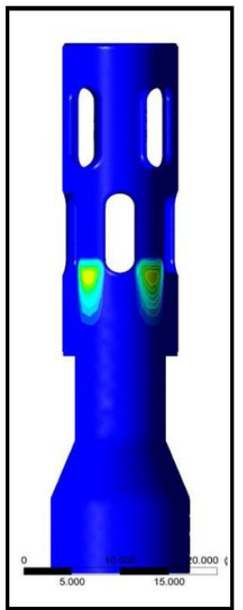

Medium

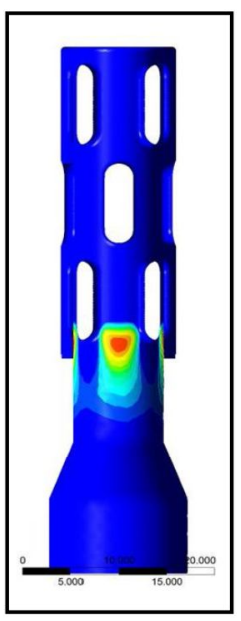

Twelve

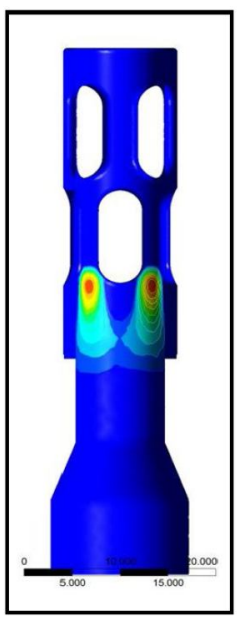

Large

FIGURE 37 - Wall shear stress for all cannulas studies. Color above blue represents a shear stress level that exceeds $0.125 \mathrm{mmHg}$ (166 dynes/cm2). The addition and size of sideholes increases the areas of high shear stress. 
To directly compare the wall shear stresses and determine the areas of high shear stress, the scales on the figures were adjusted to highlight areas higher than this threshold. Figure 37 shows all cannulas studied, the blue area represents any area under a minimum threshold of $0.124 \mathrm{mmHg}$ (166 dynes/cm2), while any color above it represents areas of higher stresses. As the number and size of the sideholes increases, the areas of higher stress increase. The maximum shear stress measured was $0.165 \mathrm{mmHg}$ (220 dynes $/ \mathrm{cm} 2)$, which is still significantly lower than the threshold for hemolysis of 1500 dynes $/ \mathrm{cm} 2$.

The levels of shear stress seen in all the cannulas are lower than the critical amount needed to activate platelets. At 150 dynes $/ \mathrm{cm} 2$, it takes $300 \mathrm{~s}$ for platelet aggregation to occur (41). The CFD flows are assumed to be during systole, so there is not enough duration of shear stress to cause activation at the calculated values levels. Even though there are differences between each design, the levels of wall shear stress remain with-in safe values for hemolysis and platelet activation for all cannula. 


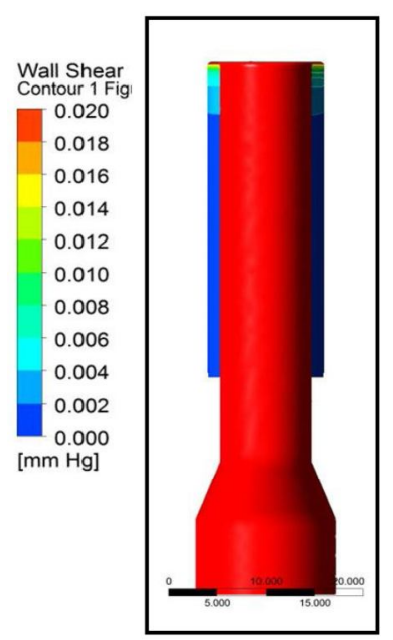

No Sideholes

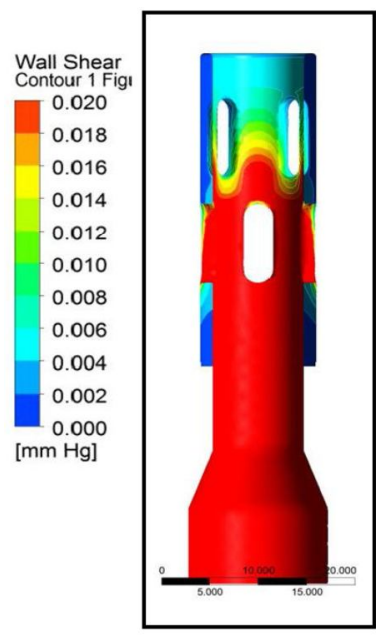

No Fillet

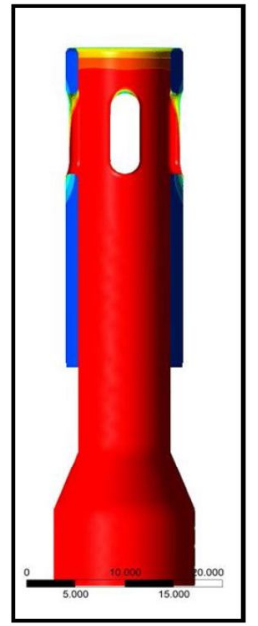

Four

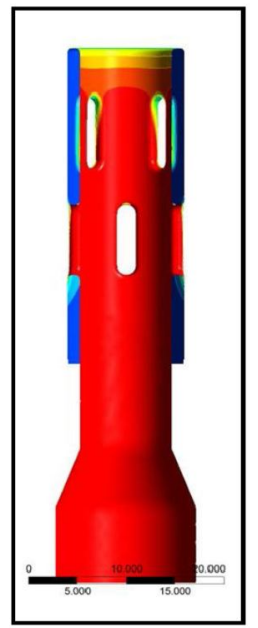

Small

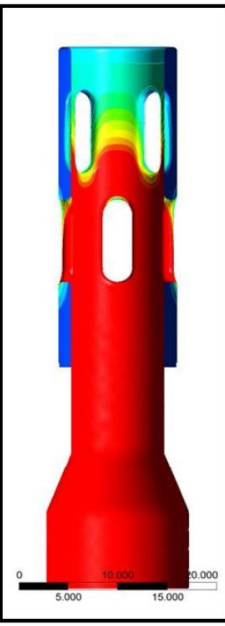

Eight

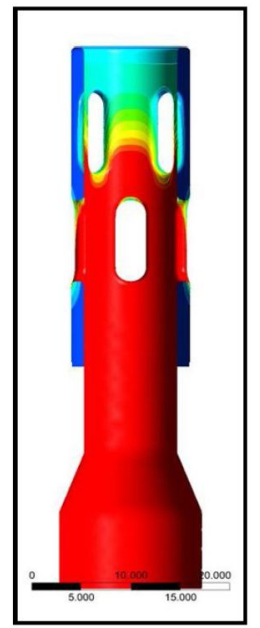

Medium

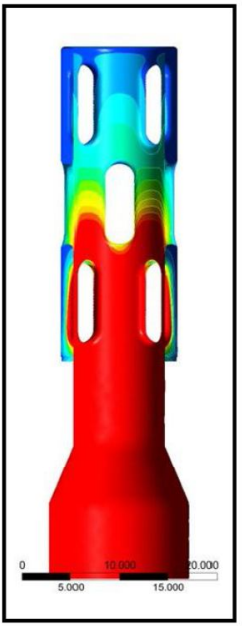

Twelve

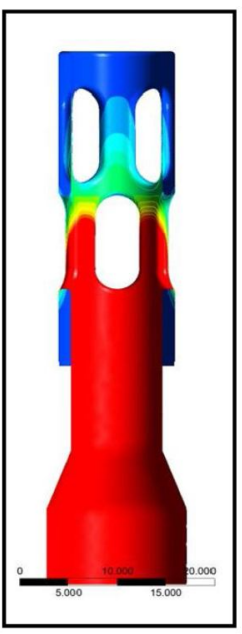

Large

FIGURE 38 - Wall shear stress for all cannulas studies. Any color below red represents a shear stress level lower than $0.02 \mathrm{mmHg}$ (27 dynes/cm2). The addition and size of sideholes increases the areas of low shear stress near the tip and proximal sideholes.

In a similar way, the areas of low shear stress were also compared for the cannulas studied. Figure 38 shows areas of lower shear stress for colors below the red threshold of $0.02 \mathrm{mmHg}$. This figure highlights the increase of lower shear stress areas as the number and size of the sideholes is increased. As more flow enters the more distal 
sideholes, the proximal sideholes have less shear energy imparted on the wall surface.

These areas could potentially lead to thrombus formation. The fluctuations between systole and diastole may help prevent thrombus formation. Looking at the velocity plots, examining the streamlines (Figure 39), and pressure field plots show flow through the proximal holes.

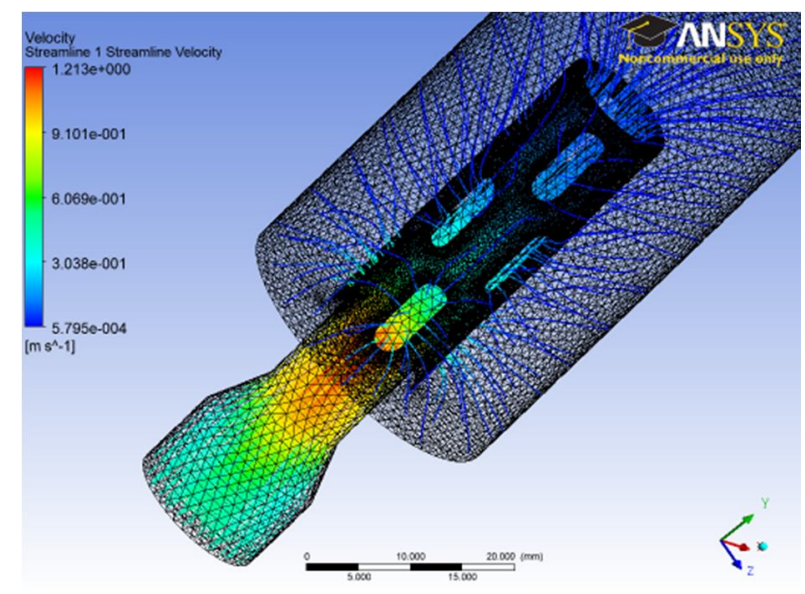

FIGURE 39 - Streamlines for 12 holes cannula

Likely just as important for this analysis is in the choice of the material used in fabrication. The nature of thrombus and platelet attachment is still an area of broad research. In vitro or animal in vivo studies are needed to properly study the effects of thrombus formation for the cannulas. The use of pyrolytic carbon and the application of the Forcefield technology described previously could greatly increase the biocompatibility of the cannula. 


\section{Effect of No Fillet}

The fillet used on all the cannulas are part of the fabrication process that smooth's out the rough edges created during fabrication. Sharp edges on the cannula can possibly damage tissue when implanted. These edges are also rounded, as it is believed that the rounded edges can help direct stream lines of flow into the cannula and reduce the concentration of high wall shear stress at the distal edges of the sideholes. Figure 40 shows a cross sectional velocity vector field for the medium and no fillet cannulas.

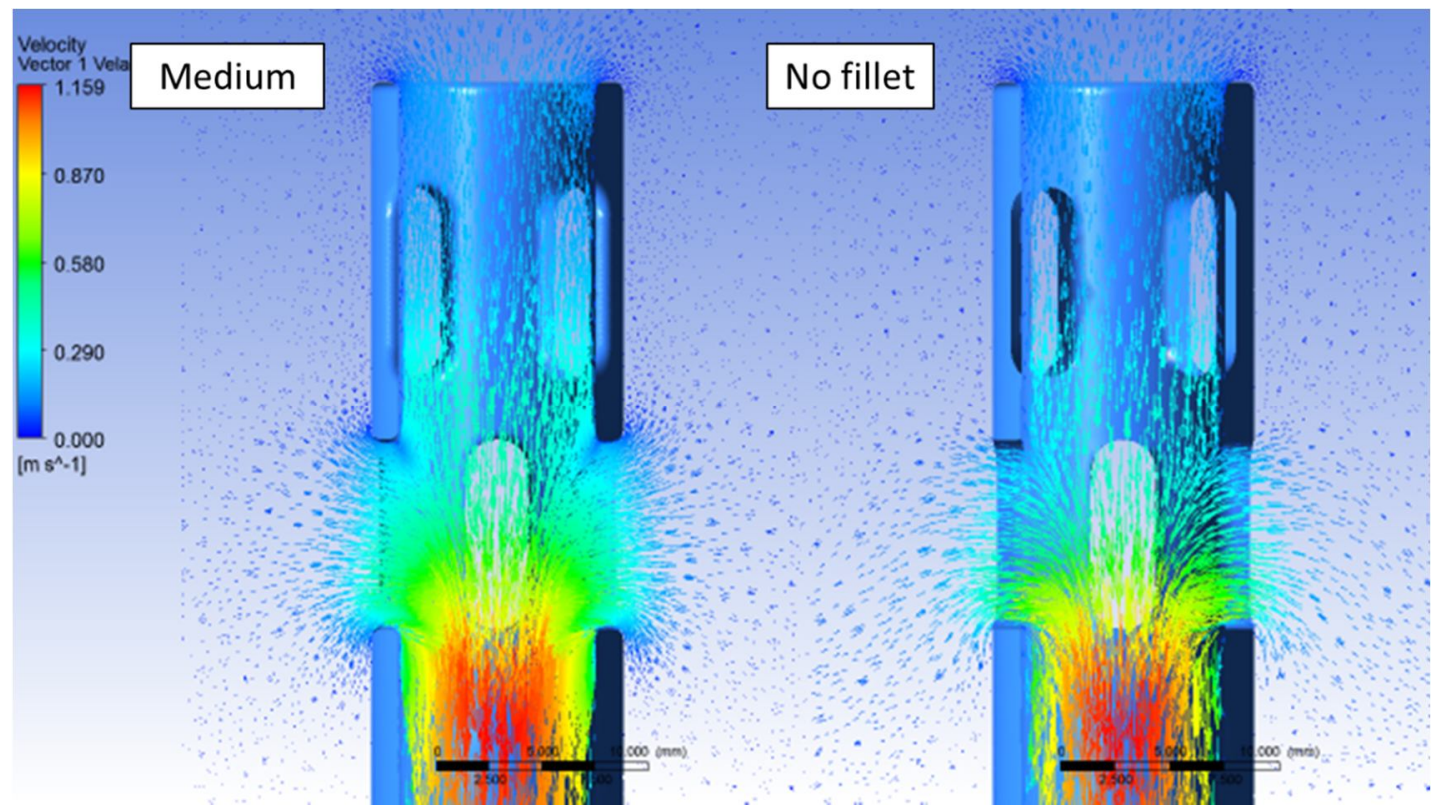

FIGURE 40 - Velocity vector fields on cross section of Medium and No Fillet cannulas.

The No Fillet Cannula had slightly higher flow and similar shear stress properties to the Medium cannula.

It can be seen in Figure 40 that the flow is indeed directed and shows a more uniform vector profile than what is seen in the no fillet cannula. The two cannulas are identical in size and geometry except for the addition of the fillet. Interestingly, the flow rate and Reynolds number are slightly higher in the no fillet cannula. Maximum shear 
stress and the distribution of high/low areas of stress do not show many differences, however higher resolution simulation of the flow over the distal edges of the sideholes may show the expected reduction in wall shear stress concentration produced by rounding these edges.

\section{Additional Benefits of AVB Technology}

Another possible benefit of the AVB system may be a brain protective advantage over aortic valve repair. Balaras et al have completed a computational model that show that cerebal blood flow is increased to the aortic head vessels even with small conduits $(10 \mathrm{~mm})$. This provides an advantage over typical aortic valve repair as there is a much higher risk for stroke during operation and postoperatively in AVR for the high risk patient groups. Figure 41 from their report shows the systolic flows for pre-operative, $20 \mathrm{~mm}$ conduit, and $10 \mathrm{~mm}$ conduit. 


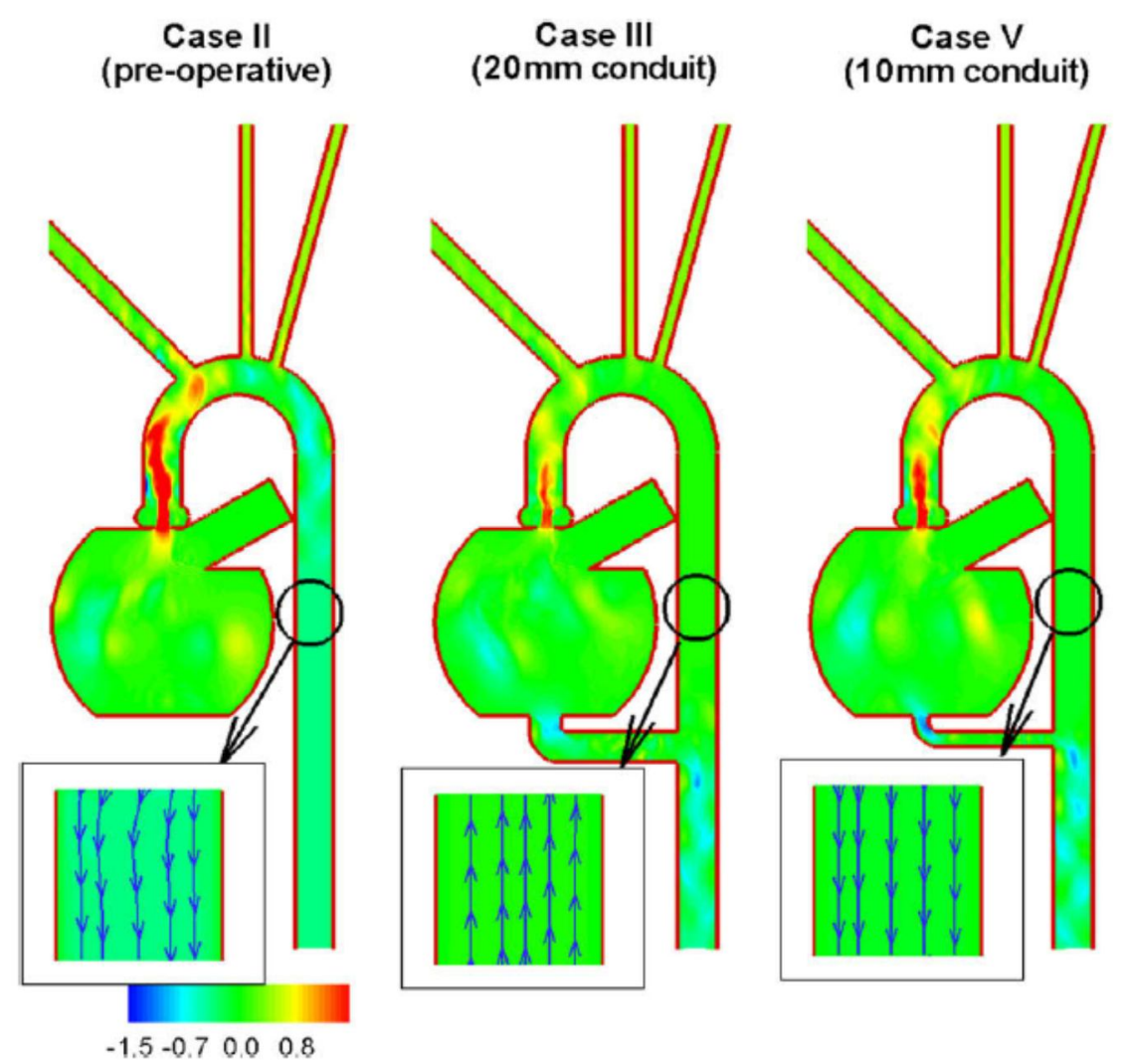

FIGURE 41 - Results from Belaras et al. Through computational modeling a brain protective advantage is presented for AVB over AVR. They also conclude that cannula as small as $10 \mathrm{~mm}$ can relieve left ventricular outflow tract obstruction in critical aortic valve stenosis (42).

The effect of AVB configuration may decrease the long-term risk of cerebral thromboembolism. The conduit provides a second path that possible clots or emboli can be shot into the peripheral vasculature instead of ending up in the cerebral vessels. They report that in all cases modeled, cerebral blood flow remained the same post-operatively from pre-operative levels (42). The brain was adequately supplied from blood ejected through the native aortic valve. The study also concluded that cannula as small as $10 \mathrm{~mm}$ 
in diameter can relieve left ventricular outflow tract obstruction in critical aortic valve stenosis.

\section{Type of Flow}

The flow is split between the native stenotic valve and conduit in an interesting fashion. Surprisingly the pressure gradients are different for these two paths. The conduit is characterized by minimal directly measured pressure gradients. Clinically the low conduit gradient has been confirmed in patients while the pressure gradient for the native aortic valve remains higher averaging $17 \mathrm{mmHg}$ (29). The difference between these gradients is almost 4 -fold.

The difference implies that the energy loss experienced by the blood element moving through the stenotic valve is much higher than the one experienced by a blood element moving through the conduit. Losses in the conduit are primarily due to shear stresses from the conduit walls and losses from the prosthetic valve as well. In contrast, losses in the native aortic valve are through kinetic energy as blood decelerates inefficiently across the valve. These losses are higher than ones due to wall stress and can be amplified in the case of turbulent, disturbed flows (29).

The difference in mean gradients is consistent with the split of flow between the two elements. Data from the dynamic flow loop, clinical data, and computational data all show this spit of approximately 60:40 in relative \% flow in the conduit and aortic valve respectively. The results suggest, as some researchers have described, that the flow split is determined largely by ventricular systolic geometries and to a small extent by the gradient across the native aortic valve (29). 


\section{Cannula Size}

The largest question in the development of this technology is: what is the smallest size that can be used to achieve adequate relief? The benefits of decreasing the conduit sizes are many with the most important being a decrease the intraoperative risk in the surgical insertion of the conduit. A smaller cannula translates into a smaller stab incision, less bleeding in left ventricular apex, and less suturing needed to secure the cannula. The currently used systems commonly use conduits and components that are $20 \mathrm{~mm}$ in diameter, though many believe that this size could be much smaller and still obtain adequate treatment of aortic stenosis. Brown and Gammie state, "Because the conduit is a second avenue for left ventricular outflow, we believe that a conduit smaller than what would normally be used for a conventional aortic valve replacement is acceptable and in most instances desirable" (23).

The tip size was chosen on basis of the experiments run and on clinical data. Most AVB apical cannulas in the past have been $20 \mathrm{~mm}$ in diameter. As the technique gains more usage though, investigators are using smaller and smaller cannula for the procedure (3). Vliek et al. recently published clinical data on hemodynamics for a number of sizes of cannulas used that ranged from $20 \mathrm{~mm}$ to $12 \mathrm{~mm}$. They found that cannula size did not significantly affect \% flow in the conduit, but that there was a relationship between cannula size and valve pressure gradient (29). Figure 42 shows the data from this report, there is a upward trend in aortic valve gradient as the cannula size decreases (Figure, Top) while there is no effect on \% flow due to cannula size (Figure, Bottom). 


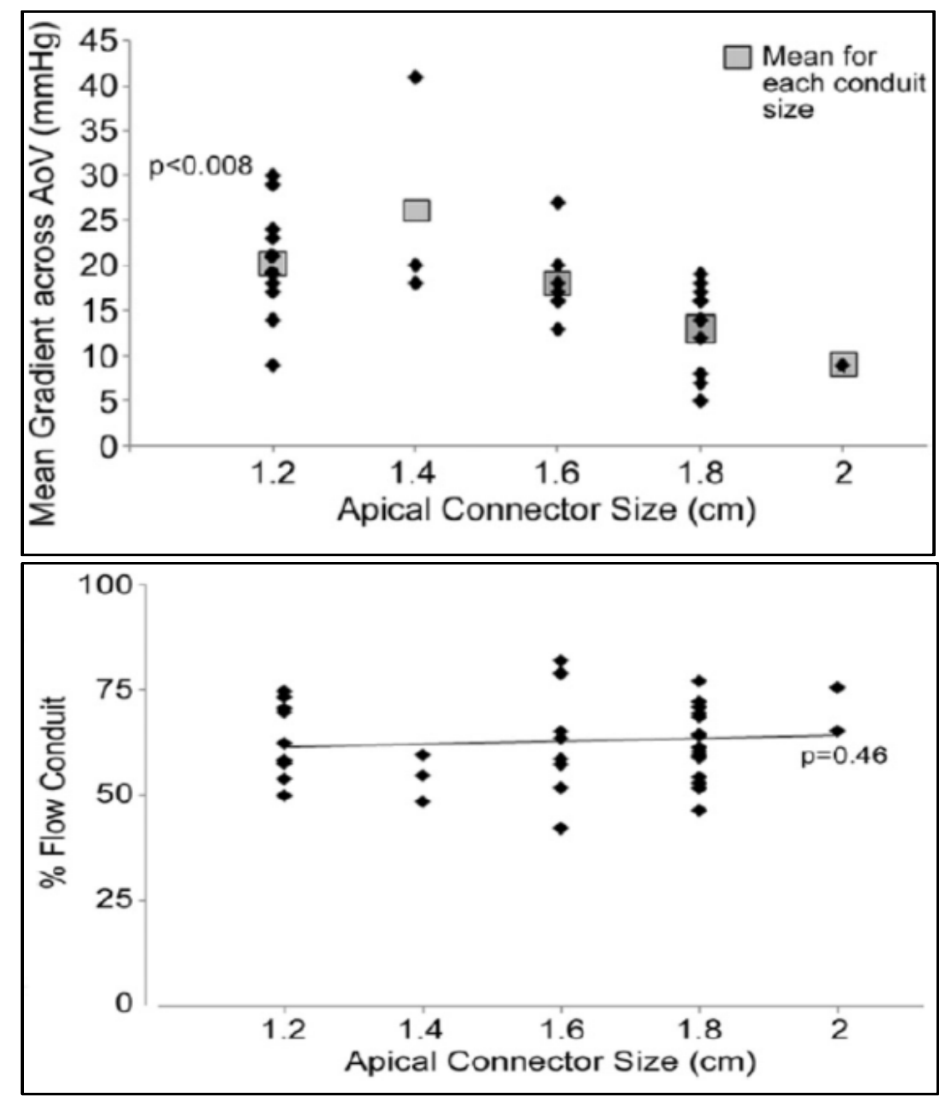

FIGURE 42 - Data from Vliek et al. showing upward trend in valve gradient as the cannula size decreases (Top) while there is no effect on $\%$ flow due to cannula size (Bottom) (29).

The mock loop studies that were performed in this thesis work, specifically the results from the preliminary prototype, show that cannula beneath $10 \mathrm{~mm}$ are not suited for use in AVB. Belarus et al. presented a computational model where 10mm cannula can effectively treat aortic stenosis though more favorable flow was seen in larger cannula, such as those presented in the mock flow loop experiments in this thesis work (42). Clinical data shows favorable results for cannula that are $12 \mathrm{~mm}$ in diameter (the smallest conduits in current use). 
Tip and Sideholes

The design of the tip was based from the results seen from the static loop testing. The open straight tip will allow for a larger amount of inflow than a tip that narrows to a smaller opening diameter. The mock loop flow tests show that a design that incorporates an open orifice at the distal most tip and inclusion of sideholes along the body is the most desired design. The use of sideholes is to decrease sidewall shear stress and maximum blood velocities to avoid hemolysis. Park et al. used a computational model to study the effect of sideholes in venous cannula. They found that flow rates in the cannula are not proportional to the number or arrangement of sideholes. They concluded though that larger number of sideholes can reduce the mean shear rate (43).

\section{CONCLUSIONS}

\section{Static Loop Testing}

In this study, a simple test to look at the most important features in the cannula tip was completed. Three different designs were investigated, which showed that diameter plays a large role in the resistance while the tip design plays a much smaller but still important role. The key findings were: (1) the resistance to flow decreases with increasing cannula diameter and (2) an "open" tip design, which is a design that incorporates an orifice at the most distal tip featuring 8 sideholes arranged in 2 sets of 4 openings, is preferred over other designs studied. Also, it demonstrated that diameter 
sizes above $10 \mathrm{~mm}(30 \mathrm{Fr})$ are favored, since smaller cannulas have a much higher resistance.

\section{Limitations}

A relatively small sample size $(\mathrm{n}=7)$ and evaluation of only three clinically used designs were included in the experimental design to demonstrate feasibility of concept. These designs were selected to highlight important characteristics of the tip design and to simplify the analysis of these cannula. Knowledge of the manufacturer's experimental methods is incomplete (proprietary intellectual property), making comparision of experimental data a challenge, though the density and viscosity of the Plasma-Lyte solution used in the current experiment is nearly identical to the water that the manufacturers used for their in vitro evaluations and should have the same flow characteristics. Some of the cannulas used for study were expired for medical use, though the flow characteristics of the cannula should be unaffected, since the expiration date relates more to the sterility of the cannula than degradation of the materials.

\section{Blood Trauma Testing}

No hemolysis, platlet activation, or blood damage observed with the prototype cannula over 6-hour test period in mock flow loop primed with bovine blood. Complete blood chemistry and plasma free hemoglobin did not vary significantly from baseline values over the entire test period. The prototype was fabricated from PYC and has 'open' tip profile, the same design that was favored by the static loop tests. This prototype also had the smallest diameter $(7 \mathrm{~mm})$ of any cannula tested in all experiments therefore 
subjecting the blood to the highest resistance of any of the designs. Even under these conditions the prototype showed excellent biocompatibility. This biocompatility could also be amplified if incorporated with the Forcefield surface alteration in future studies.

\section{Limitations}

The main limitation for this study that arise are unrelated to the device or experimental procedure, and mainly lie in the variability of the blood samples. Due to the inherent variability in blood sources, performance of in vitro studies and subsequent analysis and comparison of results can be problematic. For example, different species blood (human vs. animals) used for testing thrombogenicity show differences in mechanical fragility and rheological behavior (44).

The use of the HeartMate II VAD device requires anticoagulation, in this case heparin, to prevent possible clotting and thrombus growth inside the pump. Typical clinical application of the cannula in AVB does not use anticoagulation therapy following implantation. The HeartMate II was also only run at one fixed pump speed setting (8400rpm), creating a static flow for the duration of the experiment so the effects of blood being subjected to more physiological-like conditions was not possible. More accurate testing environment would include dynamic pressure and flow changes, representing the systolic and diastolic pressures. 


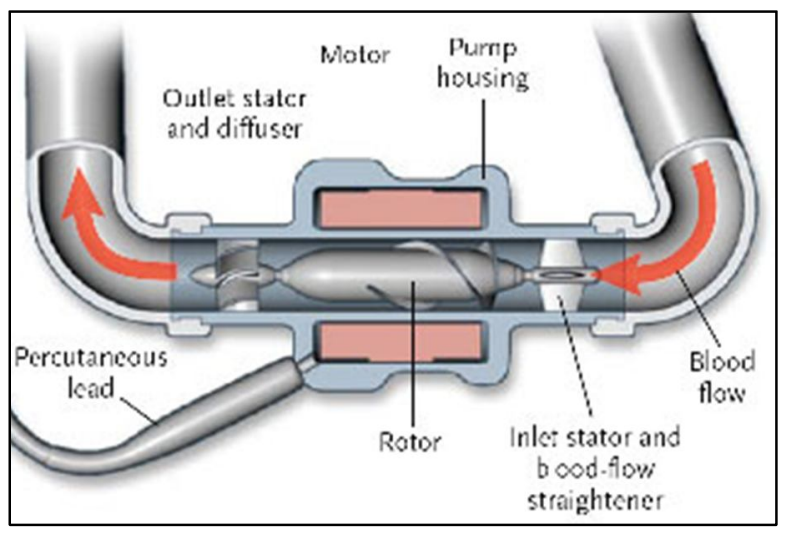

FIGURE 43 - Cross sectional view of the HeartMate II. Blood in the experiment were in contact with the small clearances inside the pump and may affect the results of the trauma study.

The HeartMate II has small clearances and two rotor bearings that contact the blood; Figure 43 shows a cross sectional view of the device. Some results from this study, specifically the changes in the bloodwork and plasma free hemoglobin, may have been the product of the HeartMate II rather than the implementation of the cannula. Clinical investigations have reported minimal $(<30 \mathrm{mg} / \mathrm{dl})$ hemolysis using these devices, though continued investigation of blood trauma associated with rotary pumps is ongoing.

\section{Dynamic Loop Testing}

The three cannulas tested in the adult circulation mock flow loop have provided a key insight to the minimal cannula diameter size, $12 \mathrm{~mm}$, which will have a clinical impact in treating aortic stenosis. The 36Fr cannula (12mm diameter) shows much better performance than the two smaller cannulas tested. The study also has shown the performance of the preliminary prototype $(7 \mathrm{~mm}$ diameter $)$ is too poor for clinical usage 
in the AVB system. The relative $\%$ conduit flow of the $36 \mathrm{Fr}$ cannula, $59 \%$, is comparable to those in current clinical use for AVB and shows an improvement of $30 \mathrm{mmHg}$ in the valve gradient for the severe AS case.

\section{Limitations}

To minimize measurement errors, post-calibrations were recorded for all pressure and flow measurements to obtain linear gain and offset calibration factors. The flow loop also used mechanical valves for the mitral, aortic, and the $19 \mathrm{~mm}$ valve used in the AVB system. These valves create a 'ringing' effect that can be seen in the waveforms, this can be especially seen in the AoP signal in the sample plots in Appendix II. This effect distorts part of the pressure waveform immediately following the closing of the valve. Comparing data using these valves to in vivo data could be flawed. Though data is averaged and peak beat-to-beat waveform data is inspected for each file, minimizing the possible error. Despite the limitations, this study provides vital information on the effect of cannula size on flow performance.

\section{Continuous Flow Dynamics Testing}

The analysis concluded that flow rate and wall shear stress increase with the addition of sideholes. Increasing the size of the sideholes decreases the shear stresses and increases flow. All wall shear stresses were significantly lower (maximum value from all designs was 220 dynes/cm2) than the threshold for hemolysis (1500 dynes/cm2) and platelet activation levels. Flow was shown to be turbulent for all cannulas tested with 
Reynolds values being slightly greater than 3,000 . The highest flow conditions were seen with the cannula with no sideholes and the cannula with large sideholes.

The cannula without sideholes showed the best blood biocompatibility in terms of ideal wall stress magnitude and area. Due to safety issues, sideholes are an important addition to the design. Increasing the number of sideholes may increase flow, but my also lead to areas of low shear stress near the tip, which may lead to thrombus formation. In vitro and/or in vivo studies are needed to further investigate these effects.

\section{Limitations}

The steady flows used in both studies do not represent the average flow rate through the cannula and only represent the flow rate during systole. Physiologic pulsatile flow may affect the velocity profiles and, therefore, the comparisons among cannula. The pressure boundary assumption was based upon a clinical median value, which averaged from 3 different sizes of cannula and represents the entire conduit as opposed to just the cannula. A different boundary pressure may be more accurate than the $6 \mathrm{mmHg}$ used in these simulations. The prosthetic valve in the conduit may create more resistance than the cannula and it is uncertain what the true pressure difference is for the cannula only.

This computational flow analysis also does not account for the left ventricular geometries that may play a role in flow characteristics. There are two paths for blood to take in this system, the aortic valve and the AVB conduit, and the shape of the ventricle may have a role in the split of flow through each. 


\section{$\underline{\text { Summary of Experimental Results }}$}

The cannula studies performed have aided in the development of the specifications for a design of an apical cannula. In early investigations, the prototype design was fabricated as a potential AVB therapy. Results from the static loop testing and specifically the dynamic loop testing proved this design to be a poor choice for application in AVB system. However, the insights learned from these studies have contributed to a design of a new apical cannula presented in the next in Section X. Table $\mathrm{XV}$ provides a summary of the results from experiments performed.

TABLE XV

SUMMARY OF RESULTS FROM EXPERIMENTAL STUDIES

\begin{tabular}{|c|c|}
\hline Test & \multicolumn{1}{c|}{ Result } \\
\hline Static Flow Loop & $\Rightarrow \begin{array}{l}\text { Resistance to flow decreases with increasing cannula diameter } \\
\text { Design that features an open distal oriface and sideholes along the } \\
\text { tip of the body favored over three designs tested }\end{array}$ \\
\hline Blood Trauma & $\Rightarrow \begin{array}{l}\text { Preliminary Prototype fabrication material shows good } \\
\text { biocompatibility }\end{array}$ \\
& $\Rightarrow \begin{array}{l}\text { No significant hemolysis or blood damage due to cannula } \\
\text { Dynamic Flow Loop }\end{array}$ \\
$\Rightarrow \begin{array}{l}\text { Preliminary Prototype developed not large enough for clinical } \\
\text { improvement of AVB }\end{array}$ \\
$\begin{array}{l}\text { Flow rate and \% conduit flow increases with cannula diameter size } \\
\text { 12mm diameter tip (36Fr) smallest cannula whose \% conduit flow } \\
\text { matches clinical data for currently used AVB systems }\end{array}$ \\
\hline CFD Analysis & $\Rightarrow \begin{array}{l}\text { Flow rate and shear stresses influenced by number and size of } \\
\text { sideholes } \\
\text { Wall shear stress below threshold for hemolysis damage and } \\
\text { platelet activation for all designs }\end{array}$ \\
\hline
\end{tabular}


These experiments have given insight for specifications of a cannula design specifically for three main design points: 1) Cannula Size, 2) Biocompatibility, 3) Tip Characteristics.

1. Cannula Size - The dynamic loop testing showed that the preliminary prototype was not large enough to be an effective treatment for AVB. The dynamic and static loop testing both showed a critical point in flow characteristics between $10 \mathrm{~mm}$ and $10.7 \mathrm{~mm}$ (30Fr and 32Fr) where the resistance increases dramatically. This is also supported by the cannula manufacturer pressure vs. flow rate data. Data from both experiments suggest using cannula $10.7 \mathrm{~mm}$ in outer diameter or larger.

2. Biocompatibility - The preliminary prototype showed good biocompatibility for use of pyrolytic carbon as a fabrication material. Both the blood trauma mock loop and CFD analysis show no hemolysis and minimal to no platelet activation. Future application of the Forcefield technology will also greatly increase the biocompatibility of the cannula.

3. Tip Characteristics - Static flow loop showed a design that incorporated an open orifice at the distal tip with sideholes was favored over other designs studied. The CFD analysis show flow rate increased with addition of sideholes while increasing the size of the sideholes decreased the shear stresses. Turbulent flow was also seen to dominate the flow during systole in all simulations. 


\section{FINAL APICAL CANNULA DESIGN}

The results of the experiments have led to the final proposed design for an apical cannula. The cannula and model for the AVB system are now presented.

\section{$\underline{\text { Apical Cannula Design }}$}

The final design for the apical cannula was developed from the results of experiments performed in this thesis work. The cannula consists of a 12mm (36French) outer diameter opening with an open tip design shown in Figure 44.

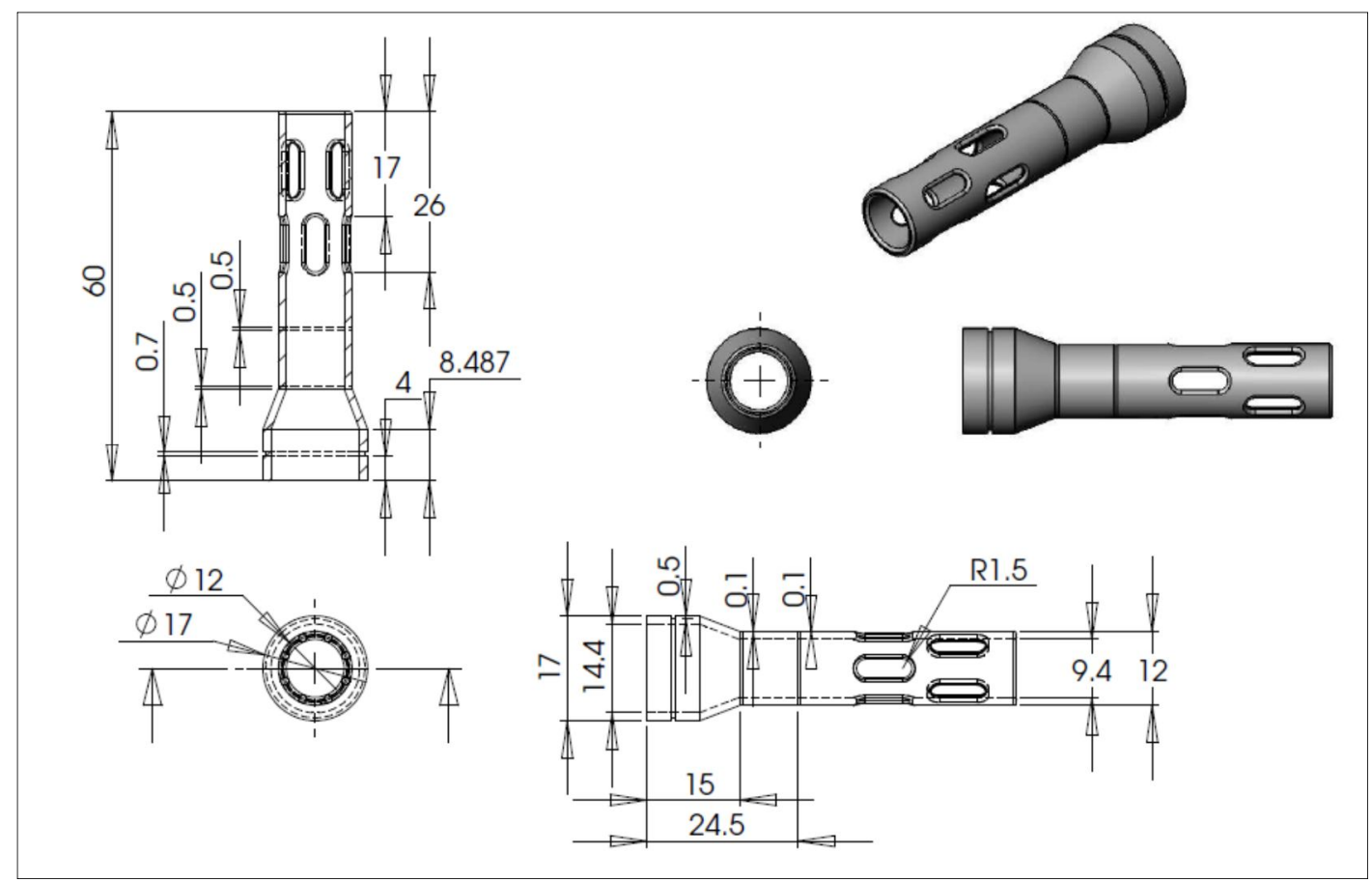

Figure 44 - Apical cannula design developed from mock loop studies created with CAD software (SolidWorks SP3.0, Dassault Systèmes). The selected criterion includes a 12mm (36Fr) outer diameter, an open tip design, and is fabricated from pyrolytic carbon. All units are in millimeters. Upper Left - Sectioned Side view, Lower Left - Top view, Lower Right - Right Sided view, Upper Right - 3D rendered drawings. 
The tip of the cannula is $12 \mathrm{~mm}$ in diameter, which adapts to a larger $17 \mathrm{~mm}$ at the base of the cannula where it attaches to the conduit material. There is a small $0.5 \mathrm{~mm}$ engraved ring at the base of the $17 \mathrm{~mm}$ section of the cannula so that graft material may be sewn onto the cannula. The light $0.1 \mathrm{~mm}$ etchings are cosmetic and intended to assist the surgeon with implantation. In a blood-soaked operation field, the etchings give a tactile feel to the cannula and can aide in implantation so the surgeon can know how far the cannula is inside the apex of the left ventricle.

The tip characteristics have a straight, open orifice at the proximal tip with eight sideholes arranged offset in two rows. These openings also feature rounded edges and are smooth to improve biocompatibility and flow characteristics. The size and number of sideholes on the cannula are the same as medium, eight sideholes model studied in the CFD analysis. The proposed cannula is the same cannula model used in the CFD studies. Appendix IV gives the results report of CFD analysis of this model.

The fabrication of the apical cannula will be identical to that used on the preliminary prototype. The thickness of the pyrolytic carbon is based from proprietary fabrication methods. The cannulas studied in static and dynamic mock flow loops all had wall thicknesses slightly larger than $1 \mathrm{~mm}$. The proposed apical cannula has a wall thickness of $1.3 \mathrm{~mm}$.

\section{Aortic Valve Bypass System}

The proposed AVB system consists of the apical cannula connected to a right angle bend adapter, graft material, and mechanical valve shown in Figure 45. The apical cannula has a $12 \mathrm{~mm}$ tip ( $36 \mathrm{Fr}$ ) that widens to $17 \mathrm{~mm}$ at the base. The cannula connects to 
$17 \mathrm{~mm}$ Dacron graft material wire reinforced to conform to a right angle bend. This conduit has a $17 \mathrm{~mm}$ pyrolytic mechanical valve. The conduit is $80 \mathrm{~mm}$ in length, which is the best approximation for the distance between the left ventricular apex to the descending aorta in an average patient.

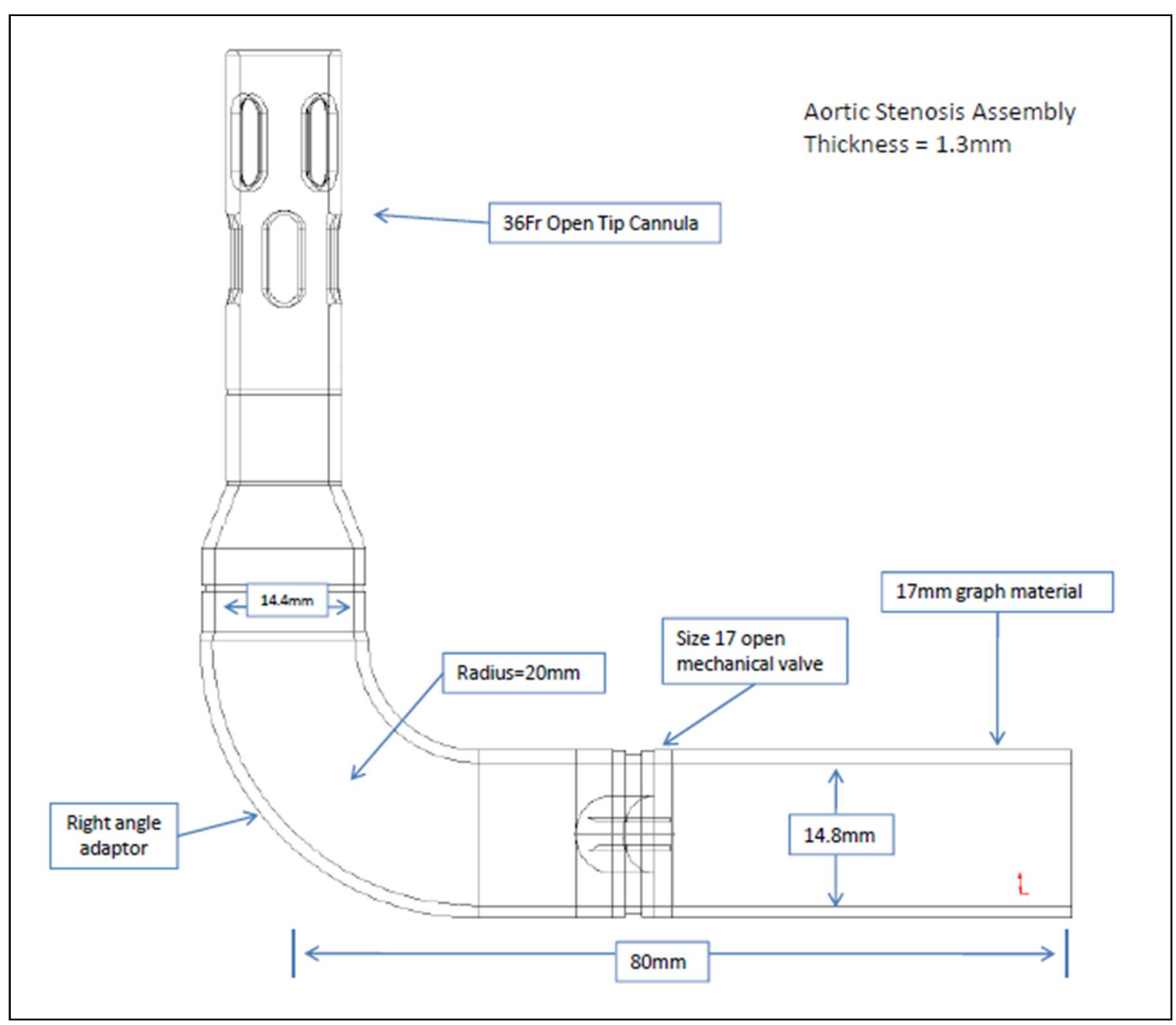

FIGURE 45 - Proposed design for the AVB system. The apical cannula adapts into a right bend adaptor and into a size $17 \mathrm{~mm}$ Dacron graph. The valve intended for use is a mechanical valve also sized to $17 \mathrm{~mm}$. This model was designed through use of CAD software (SolidWorks SP3.0, Dassault Systèmes) and used in CFD analysis. 


\section{Closing Comments}

This thesis has presented the possible advantages that aortic valve bypass has over aortic valve repair in high risk patient groups. The experiments performed have been used to develop a new design for the apical cannula component in the aortic valve bypass system. This incorporates new technologies, techniques, and fabrication materials. The 'forcefield' technology and Seldinger insertion technique are exciting prospects to make this design feasible for market development.

Aortic valve bypass results in a low gradient across the native stenotic valve and a small gradient across the conduit. The results from the mock loop experiments, CFD analysis, clinical data, and findings from other researchers have aided in the creation of a new apical cannula. It is hoped that this design will be a more favorable design over currently used apical connectors. Further studies are needed to validate this design.

\section{Future Work}

Future work on this system would include experimental analysis on the proposed cannula. It will need to be fabricated and tested against currently available cannula and apical connectors using methods similar to this thesis work. In vivo animal model studies would aide in biocompatible and ultimate efficacy of the AVB system. In addition, future studies may further investigate the shape of the sideholes. For this study only oval shaped sideholes with $0.5 \mathrm{~mm}$ fillet were studied. There may be alternate geometries that could improve flow characteristics. 


\section{LIST OF REFERENCES}

1. Alpert JS: Aortic stenosis: A new face for an old disease. Arch Intern Med 2003; 163:1769

2. Yeo KK, Low RI. Aortic stenosis: assessment of the patient at risk. Journal of Interventional Cardiology 2007;20(6):509-16

3. Gammis JS, Krowsoski LS, Brown JM, Odonkor PN, Young CA, Santos MJ, Gottdiener JS, Griffith BP. Aortic Valve Bypass Surgery: Midterm Clinical Outcomes in a High-Risk Aortic Stenosis Population. Circulation 2008;118:146066

4. Carrel A. On the experimental surgery of the thoracic aorta and heart. Ann Surg 1910;52:83-95.

5. Vassiliades TA Jr. Off-pump apicoaortic conduit insertion for high-risk patients with aortic stenosis. Eur J Cardiothorac Surg 2003;23:156-8.

6. Slaughter MS, Pederson B, Graham JD, Sobieski MA, Pappa PS, Tatooles AJ. Evaluation of New "Forcefield"TM Technology: Reducing Platelet Adhesion and Cell Coverage of Pyrolytic Carbon Surfaces. Presented as slide presentation ASAIO’s 54th Annual Conference, San Francisco June, 2008

7. Pantalos GM, Koenig SC, Gillars KJ, Giridharan GA, Ewert DL. Characterization of an adult mock circulation for testing cardiac support devices. ASAIO J. 2004 Jan-Feb;50(1):37-46.

8. Mihaljevic T, Sayeed M R, Stamou S C, Paul S. Pathophysiology of Aortic Valve Disease. Cohn Lh, ed. Cardiac Surgery in the Adult. New York: McGraw-Hill, 2008; 825-840.

9. Zigelman C.Z., Edelstein P.M. Aortic Valve Stenosis. Anesthesiology Clin 2009;27:519-32.

10. Carabello BA. Aortic stenosis. N Engl J Med 2002;346(9):677-82.

11. Nkomo VT, Gardin JM, Skelton TN, Gottdiener JS, Scott CS, Sarano ME. Burden of valvular heart diseases: a population-based study. Lancet 2006;368:1005-11 
12. Bonow RO, Carabello BA, Chatterjee K, et al. ACC/AHA 2006 guidelines for the management of patients with valvular heart disease: a report of the American College of Cardiology/American Heart Association Task Force on Practice Guidelines (writing committee to develop guidelines for the management of patients with valvular heart disease). Circulation 2006;114:e84-231.

13. Rosenhek R, Binder T, Porenta G, et al. Predictors of outcome in severe, asymptomatic aortic stenosis. N Engl J Med 2000; 343:611-7.

14. Pellikka PA, Sarano ME, Nishimura RA, et al. Outcome of 622 adults with asymptomatic hemodynamically significant aortic stenosis during prolonged follow-up. Circulation 2005;111:3290-5.

15. Grunkemeier GL, Li HH, Starr A. Heart valve replacement: a statistical review of 35 years' results. J Heart Valve Dis 1999;8:466 -71.

16. Blackstone EH, Cosgrove DM, Jamieson WRE, Birkmeyer NJ, Lemmer JH, Miller DC, Butchart EG, Rizzoli G, Yacoub M, Chai A. Prosthesis size and long-term survival after aortic valve replacement. J Thorac Cardiovasc Surg 2003;126:783-96

17. Roberts WC, Ko JM, Garner WL, et al. Valve structure and survival in octogenarians having aortic valve replacement for aortic stenosis (+/- aortic regurgitation) with versus without coronary artery bypass grafting at a single US medical center (1993-2005). Am J Cardiol 2007;100:489-95.

18. Freeman RV, Otto CM. Spectrum of calcific aortic valve disease: pathogenesis, disease progression and treatment strategies. Circulation 2005;111:3316-26.

19. Sarnoff SJ, Donovan TJ, Case RB. The surgical relief of aortic stenosis by means of apical-aortic vavular anastomosis. Circulation 1955;11:564-75

20. Brown JW, Girod DA, Hurwitz RA, et al. Apricoaortic valved conduits for complex left ventricular outflow obstruction: technical considerations and current status. Ann Thorac Surg 1984;38:162-8.

21. McCarthy P. Invited commentary: Aortic valve bypass for the high risk patient with aortic stenosis. Ann Thorac Surg 2006;81:1610-11

22. Gammis JS, Brown JW, Brown JM, et al. Aortic valve bypass for the high risk patient with aortic stenosis. Ann Thorac Surg 2006;81:1605-11.

23. Brown JW, Gammie JS. Off-Pump Aortic Valve Bypass Using a Valved ApicalAortic Conduit. Operative Techniques in Thoracic and Cardiovascular Surgery. 2007 
24. Cooley PA, Lopez RM, Absi TS: Apicoaortic conduit for left ventricular outflow tract obstruction: revisited. Ann Thorac Surg 69:1511-1514, 2000

25. Giovanni Speziali, MD, and Kenton J. Zehr. Left Ventricle Apical-Aortic Conduit for Aortic Stenosis. Operative Techniques in Thoracic and Cardiovascular Surgery. 2007

26. Seldinger S. Catheter Replacement of the Needle in Percutaneous Arteriography: A new technique. Acta Radiologica 1953; 39(5):368-76

27. ATSM Standard F1830-97, 2005, "Standard Practice for the Selection of Blood for In Vitro Evaluation of blood pumps", ASTM International, West Conshohocken, PA, 2005, DOI: 10.1520/F1830-97R05, www.astm.org

28. ISO 7199, 2009, "Cardiovascular implants and artificial organs - Blood-gas exchangers (oxygenators)", International Organization for Standardization, Geneva, Switzerland. www.iso.org

29. Vliek, Crystal J., Balaras, Elias, Li, Shuying, Lin, Jolinta Y., Young, Cindi A., DeFilippi, Christopher R., Griffith, Bartley P., Gammie, James S. Early and Midterm Hemodynamics After Aortic Valve Bypass (Apicoaortic Conduit) Surgery. Ann Thorac Surg 2010 90: 136-143

30. Koenig SC, Woolard C, Drew G, Unger L, Gillars K, Ewert D, Gray L, Pantalos G. Integrated Data Acquisition System for Medical Device Testing and Physiology Research in Compliance with Good Laboratory Practices. Biomed Instrum Technol. 2004; 38(3):229-40.

31. Drew GA and SC Koenig. Biomedical patient monitoring, data acquisition, and playback with LabVIEW. Chapter 2 (pp 92-98): In LabVIEW for Automotive, Telecommunications, Semiconductor, Biomedical, and other Applications. Prentice Hall PTR, Upper Saddle River, NJ, 2000.

32. Drew, G. A. and Koenig, S. C., "Biomedical Patient Monitoring, Data Acquisition, and Playback with LabVIEW®," in Virtual Bio-Instrumentation: Biomedical, Clinical, and Healthcare Applications in LabVIEW@, Olansen, J. B. and Rosow, E., 180-186, Prentice Hall, 2002.

33. Brookshier K A; Tarbell J M. Evaluation of a transparent blood analog fluid: aqueous xanthan gum/glycerin. Biorheology 1993;30(2):107-16.

34. Schroeder MJ, Perreault B, Ewert DL, Koenig SC. HEART: an automated beat-tobeat cardiovascular analysis package using Matlab. Comput Biol Med. 2004 Jul;34(5):371-88. 
35. Terumo Cardiovascular Systems. Cannulae, Catheters and Sternotomy Products US Product Catalog, June 2008. Terumo Cardiovascular Systems Corporation 6200 Jackson Road Ann Arbor, Michigan 48103-9300 USA.

36. Behr M, Arora D, Coronado O, Pasquali M. Models and finite element techniques for blood flow simulation. Int J Comp Fluid Dyn 2006;20:175-81.

37. McIntire LV. Guidelines for Blood-Material Interactions. Report of the National Heart, Lung, and Blood Institute Working Group. U. S. Department of Health and Human Services, Public Health Service. Bethesda, MD: NIH publication, 1985.

38. Balasubramanian V, Slack SM. The effect of fluid shear and co-adsorbed proteins on the stability of immobilized fibrinogen and subsequent platelet interactions. J Biomater Sci Polym Ed 2002;13:543-61.

39. L.B. Leverett, J.D. Hellums, C.P. Alfrey and E.C. Lynch. Red Blood Cell Damage by Shear Stress. Biophys J. 1972;12(3):257-73.

40. Sutera SP, Mejhardi MH. 1975. Deformations and fragmentation of human red blood cells in turbulent shear flow. Biophys J. 1975;15:1

41. Ramstack JM, Zuckerman L, Mockros, LF. Shear induced activation of platelets. J. Biomech 1979;12:113

42. Belaras E, Cha KS, Griffith BP, Gammie JS. Treatment of aortic stenosis with aortic valve bypass (apicoaortic conduit) surgery: An assessment using computational modeling. J Thorac Carddiovasc Surg 2009;137:680-7

43. Park JY, Park CY, Min BG. A numerical study on the effect of side hole number and arrangement in venous cannulae. J of Biomechanics 2007; 40(5):1153-57.

44. Blackshear PL Jr, Forstrom RJ. Comparative mechanical blood properties. DHEW Publication 1973;72:525-539. 


\section{APPENDIX I}

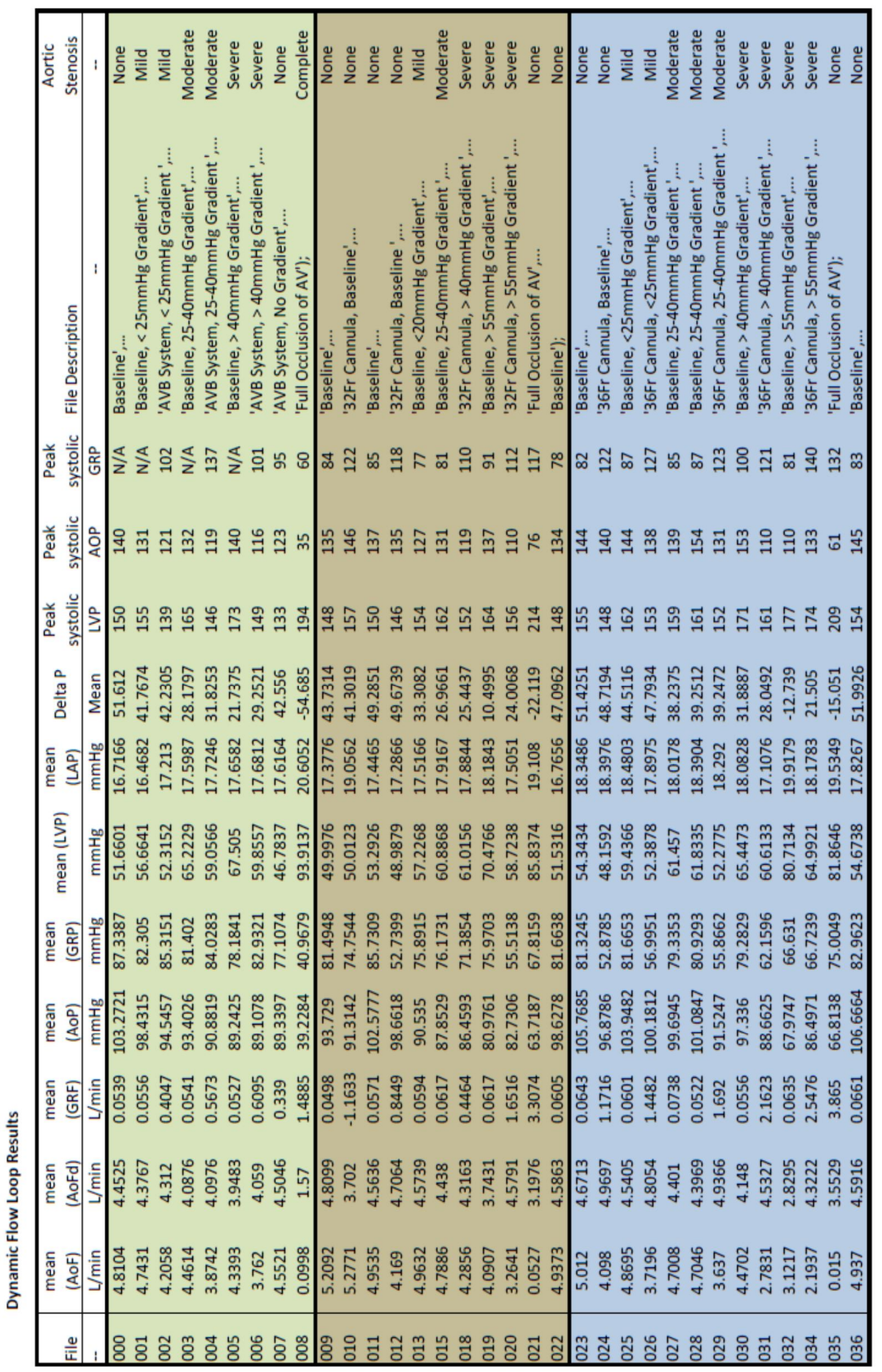




\section{APPENDIX II}
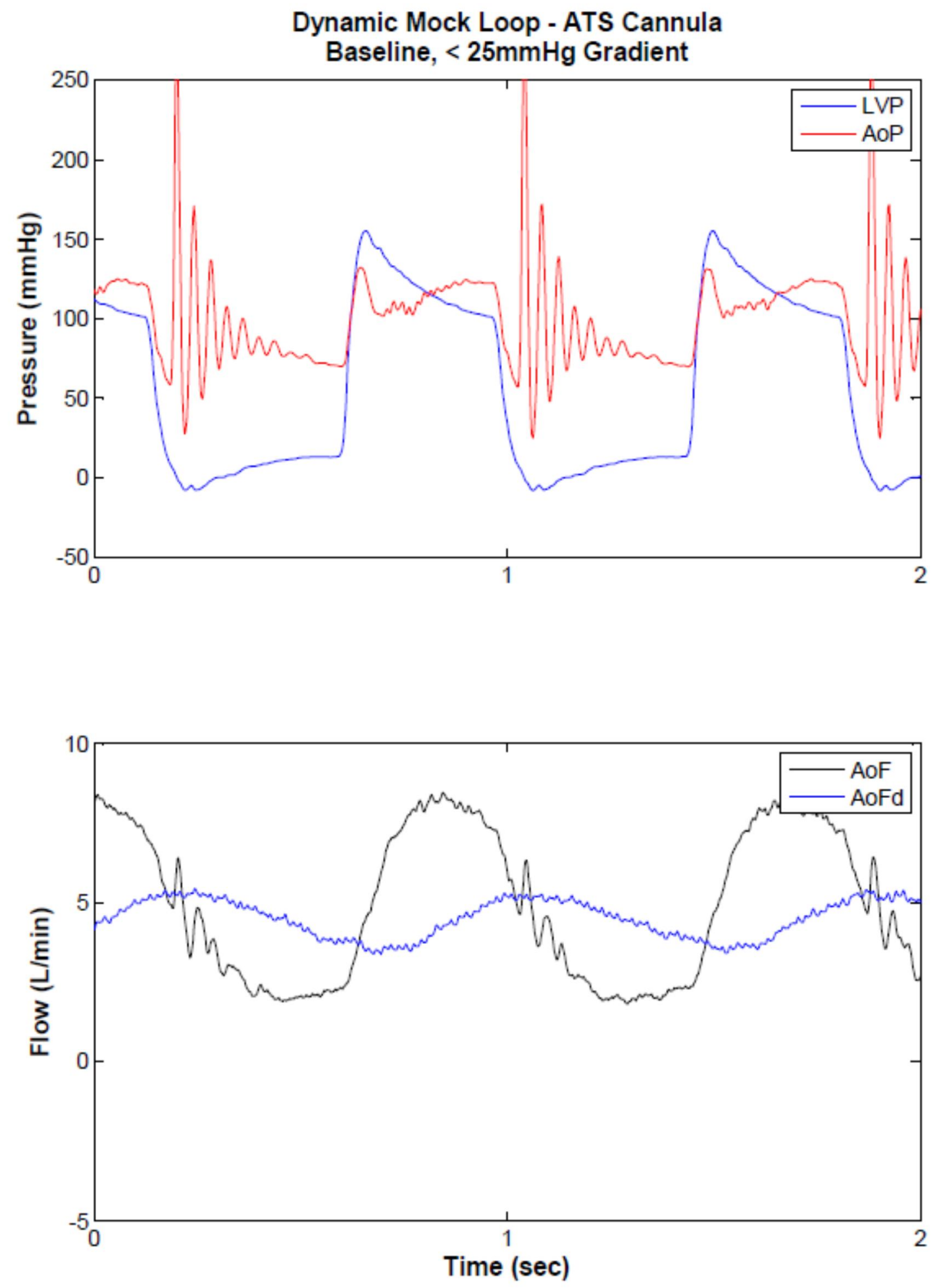


\section{APPENDIX II}
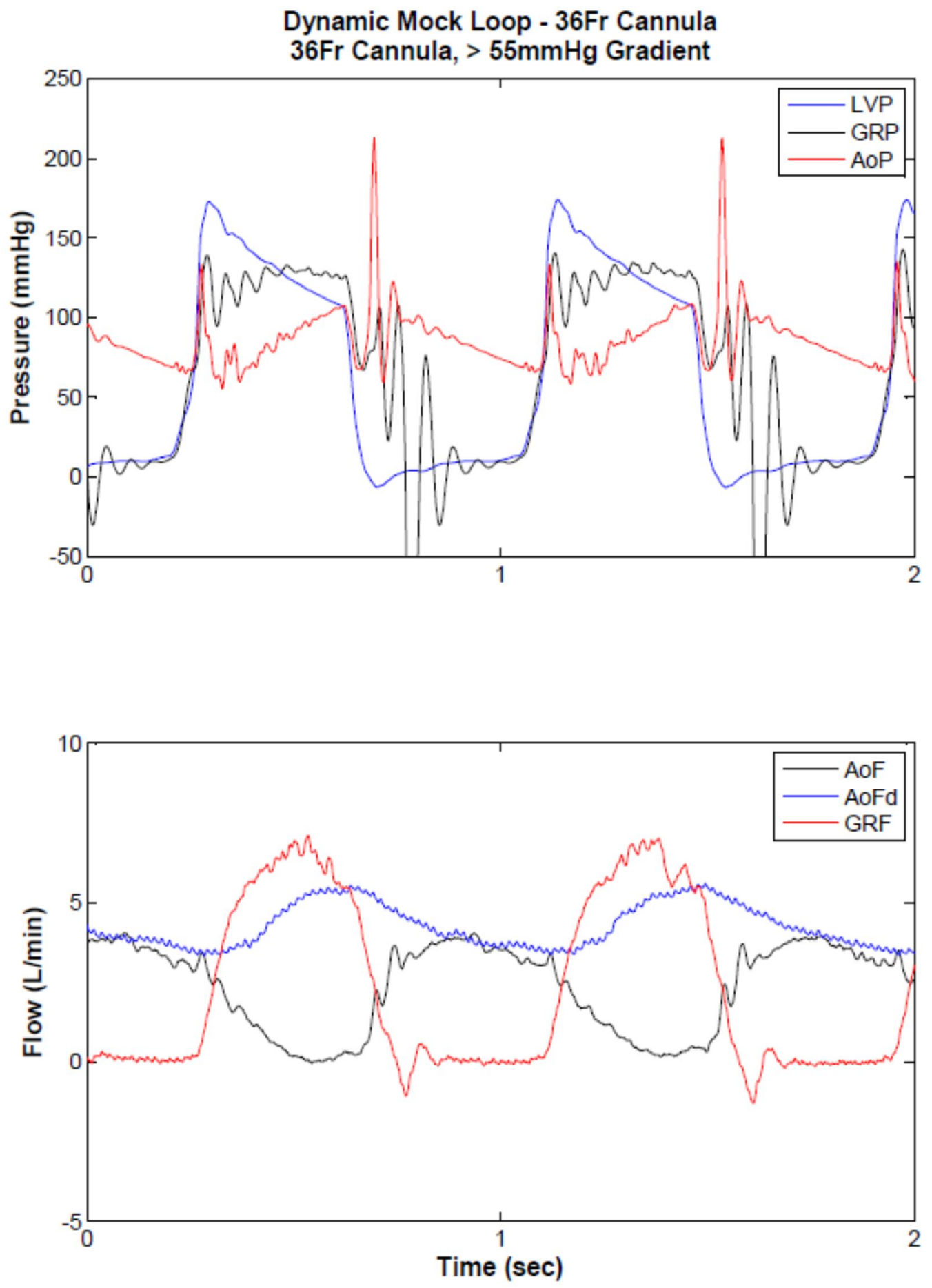
APPENDIX III

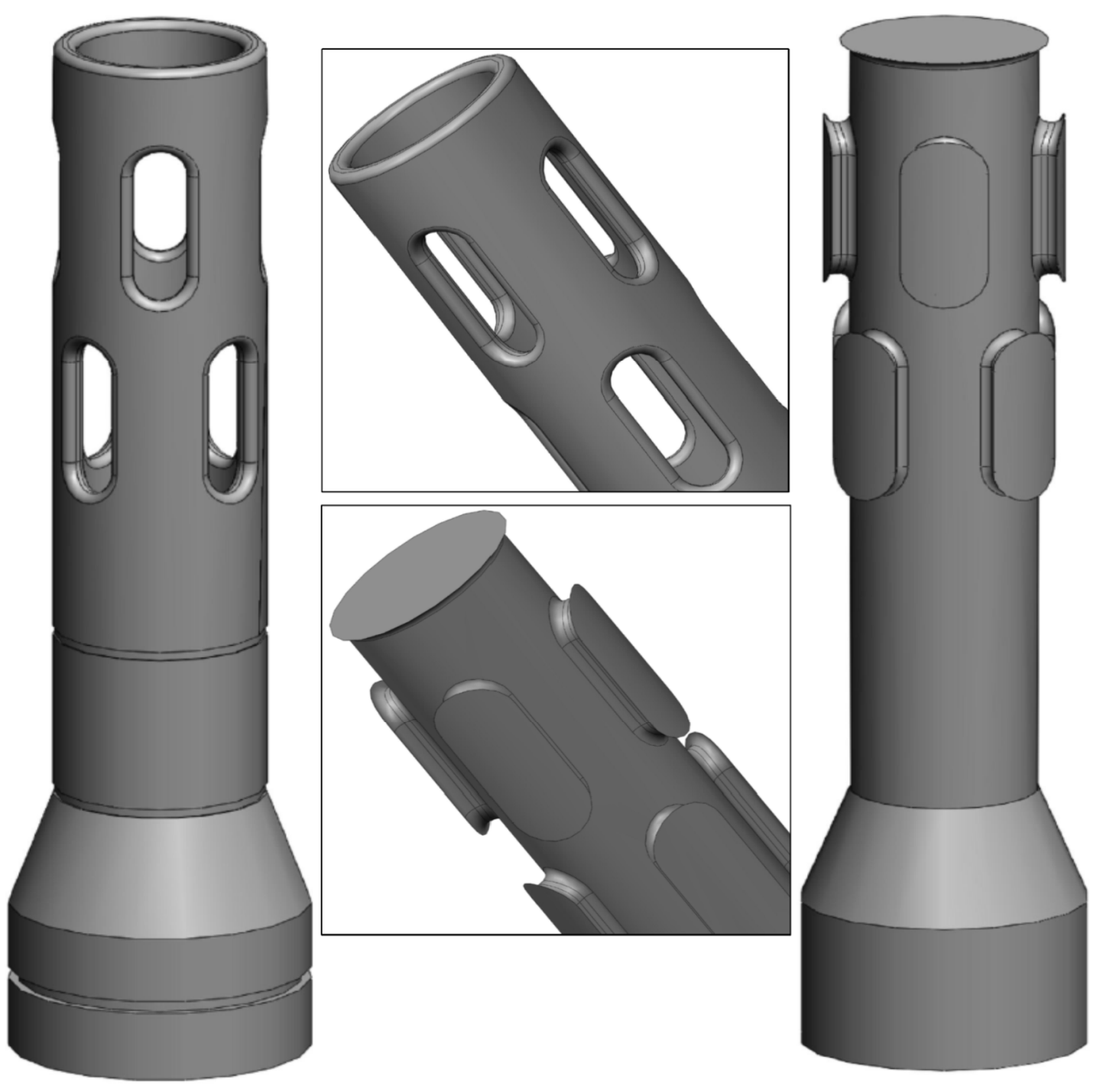




\section{APPENDIX IV}

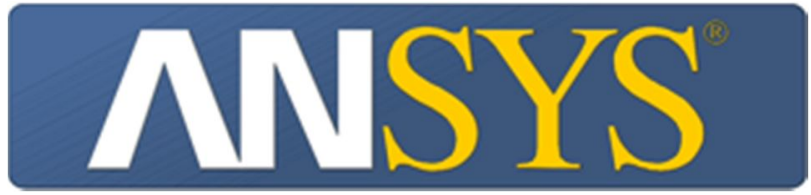

Date

2010/12/02 18:04:28

\section{Contents}

1. File Report

Table 1 File Information for CFX

2. Mesh Report

Table 2 Mesh Information for CFX

3. Physics Report

Table 3 Domain Physics for CFX

Table 4 Boundary Physics for CFX

4. Solution Report

Table 5 Boundary Flows for CFX

Table 6

Figure 1

Figure 2

Figure 3

Figure 4

Figure 5 Inside wall shear stress

Figure 6 Outside wall shear stress

Figure 7

Figure 8

Figure 9

Figure 10

5. User Data

Table 7

Figure 11

\section{File Report}

Table 1. File Information for CFX

\begin{tabular}{||l|l||}
\hline Case & CFX \\
\hline $\begin{array}{l}\text { File } \\
\text { Path }\end{array}$ & $\begin{array}{l}\text { C:IUsers } \\
\text { 037.res }\end{array}$ \\
\hline $\begin{array}{l}\text { File } \\
\text { Date }\end{array}$ & 02 December 2010 \\
\hline $\begin{array}{l}\text { File } \\
\text { Time }\end{array}$ & 05:58:51 PM \\
\hline File & CFX5 \\
\hline
\end{tabular}




\begin{tabular}{|l||l||}
\hline Type & \\
\hline File & \\
Versio & 12.0 \\
n & \\
\hline
\end{tabular}

\section{Mesh Report}

Table 2. Mesh Information for CFX

\begin{tabular}{|l|l|l|}
\hline Domain & Nodes & Elements \\
\hline Default Domain & 149169 & 814855 \\
\hline
\end{tabular}

\section{Physics Report}

Table 3. Domain Physics for CFX

\begin{tabular}{|c|c|}
\hline \multicolumn{2}{|l|}{ Domain - Default Domain } \\
\hline Type & Fluid \\
\hline Location & B1117 \\
\hline \multicolumn{2}{|l|}{ Materials } \\
\hline \multicolumn{2}{|l|}{ Blood } \\
\hline Fluid Definition & Material Library \\
\hline Morphology & Continuous Fluid \\
\hline \multicolumn{2}{|l|}{ Settings } \\
\hline Buoyancy Model & Non Buoyant \\
\hline Domain Motion & Stationary \\
\hline Reference Pressure & $1.0000 \mathrm{e}+00[\mathrm{~atm}]$ \\
\hline Turbulence Model & k epsilon \\
\hline Turbulent Wall Functions & Scalable \\
\hline
\end{tabular}

Table 4. Boundary Physics for CFX

\begin{tabular}{|c|c|c|}
\hline Domain & \multicolumn{2}{|l|}{ Boundaries } \\
\hline \multirow{7}{*}{$\begin{array}{l}\text { Default } \\
\text { Domain }\end{array}$} & \multicolumn{2}{|c|}{ Boundary - Inlet 1} \\
\hline & Type & INLET \\
\hline & Location & F1118.1117, F1132.1117, F1133.1117 \\
\hline & \multicolumn{2}{|l|}{ Settings } \\
\hline & $\begin{array}{l}\text { Flow } \\
\text { Direction }\end{array}$ & Zero Gradient \\
\hline & Flow Regime & Subsonic \\
\hline & $\begin{array}{l}\text { Mass And } \\
\text { Momentum }\end{array}$ & Static Pressure \\
\hline
\end{tabular}




\begin{tabular}{|c|c|}
\hline $\begin{array}{l}\text { Relative } \\
\text { Pressure }\end{array}$ & 5.0000e+01 [mm Hg] \\
\hline Turbulence & Medium Intensity and Eddy Viscosity Ratio \\
\hline \multicolumn{2}{|c|}{ Boundary - Outlet } \\
\hline Type & OUTLET \\
\hline Location & F1177.1117 \\
\hline \multicolumn{2}{|l|}{ Settings } \\
\hline Flow Regime & Subsonic \\
\hline $\begin{array}{l}\text { Mass And } \\
\text { Momentum }\end{array}$ & Static Pressure \\
\hline \begin{tabular}{|l} 
Relative \\
Pressure
\end{tabular} & $4.4000 \mathrm{e}+01[\mathrm{~mm} \mathrm{Hg}]$ \\
\hline \multicolumn{2}{|c|}{ Boundary - Default Domain Default } \\
\hline Type & WALL \\
\hline Location & 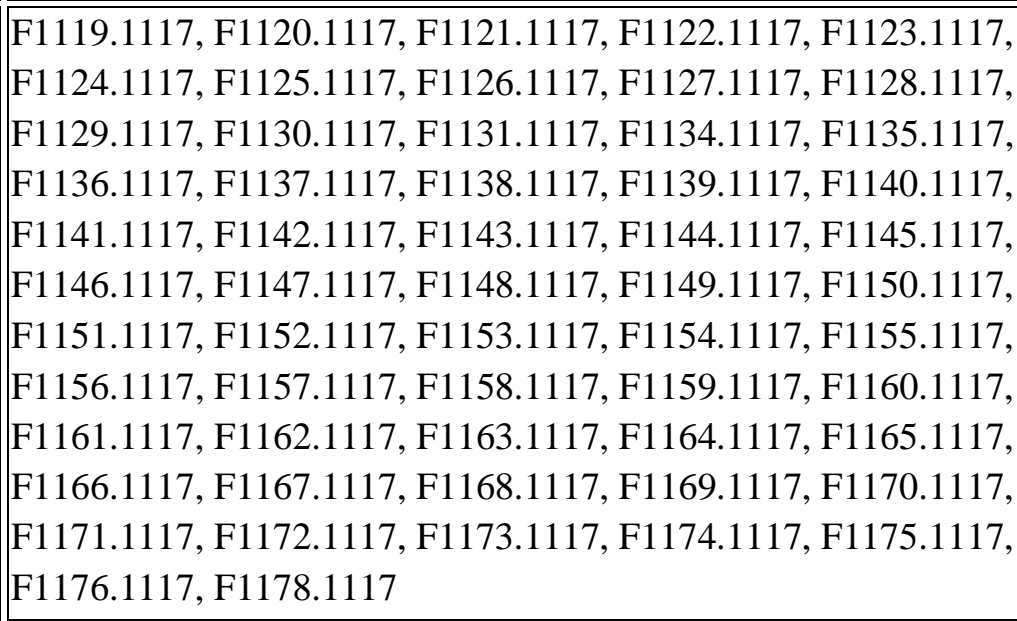 \\
\hline \multicolumn{2}{|l|}{ Settings } \\
\hline $\begin{array}{l}\text { Mass And } \\
\text { Momentum }\end{array}$ & No Slip Wall \\
\hline $\begin{array}{l}\text { Wall } \\
\text { Roughness }\end{array}$ & Smooth Wall \\
\hline
\end{tabular}

\section{Solution Report}

Table 5. Boundary Flows for CFX

\begin{tabular}{|c|c|c|c|c|c|}
\hline \multirow{2}{*}{ Location } & \multirow{2}{*}{ ||Type } & \multirow{2}{*}{ Mass Flow } & \multicolumn{3}{|l|}{ Momentum } \\
\hline & & & $\mathrm{X}$ & $\mathrm{Y}$ & $\mathrm{Z}$ \\
\hline Default Domain Default & Boundary & $0.0000 \mathrm{e}+00$ & $2.0251 \mathrm{e}-05$ & $-2.5772 \mathrm{e}-01$ & $8.6553 \mathrm{e}-05$ \\
\hline Inlet 1 & Boundary & $3.9935 \mathrm{e}-02$ & $1.4347 \mathrm{e}-04$ & $-7.2421 \mathrm{e}-01$ & $2.9014 \mathrm{e}-04$ \\
\hline
\end{tabular}




\begin{tabular}{|l|l|l|l|l|l|l|}
\hline Outlet & Boundary & $-7.0625 \mathrm{e}-02$ & $-2.8056 \mathrm{e}-06$ & $9.7880 \mathrm{e}-01$ & $-1.7091 \mathrm{e}-06$ \\
\hline
\end{tabular}

Table 6.

\begin{tabular}{|c|c|c|c|}
\hline & Average Velocity & Max Velocity & Min Velocity \\
\hline Inlet & $1.868 \mathrm{e}-02\left[\mathrm{~m} \mathrm{~s}^{\wedge}-1\right]$ & $4.994 \mathrm{e}-02\left[\mathrm{~m} \mathrm{~s}^{\wedge}-1\right]$ & $4.261 \mathrm{e}-03\left[\mathrm{~m} \mathrm{~s}^{\wedge}-1\right]$ \\
\hline Outlet & $3.912 \mathrm{e}-01\left[\mathrm{~m} \mathrm{~s}^{\wedge}-1\right]$ & $4.502 \mathrm{e}-01\left[\mathrm{~m} \mathrm{~s}^{\wedge}-1\right]$ & $3.589 \mathrm{e}-01\left[\mathrm{~m} \mathrm{~s}^{\wedge}-1\right]$ \\
\hline & Flow & Flow [liter^ $\left.1 \min ^{\wedge}-1\right]$ & \\
\hline Inlet & $1.123 \mathrm{e}-04\left[\mathrm{~m}^{\wedge} 3 \mathrm{~s}^{\wedge}-1\right]$ & $6.741 \mathrm{e}+00$ & \\
\hline Outlet & $6.344 \mathrm{e}-05\left[\mathrm{~m}^{\wedge} 3 \mathrm{~s}^{\wedge}-1\right]$ & $3.806 \mathrm{e}+00$ & \\
\hline & & & \\
\hline & & & \\
\hline & & & \\
\hline & Reynolds Number & & \\
\hline Outlet & $3.096 \mathrm{e}+03$ & & \\
\hline
\end{tabular}

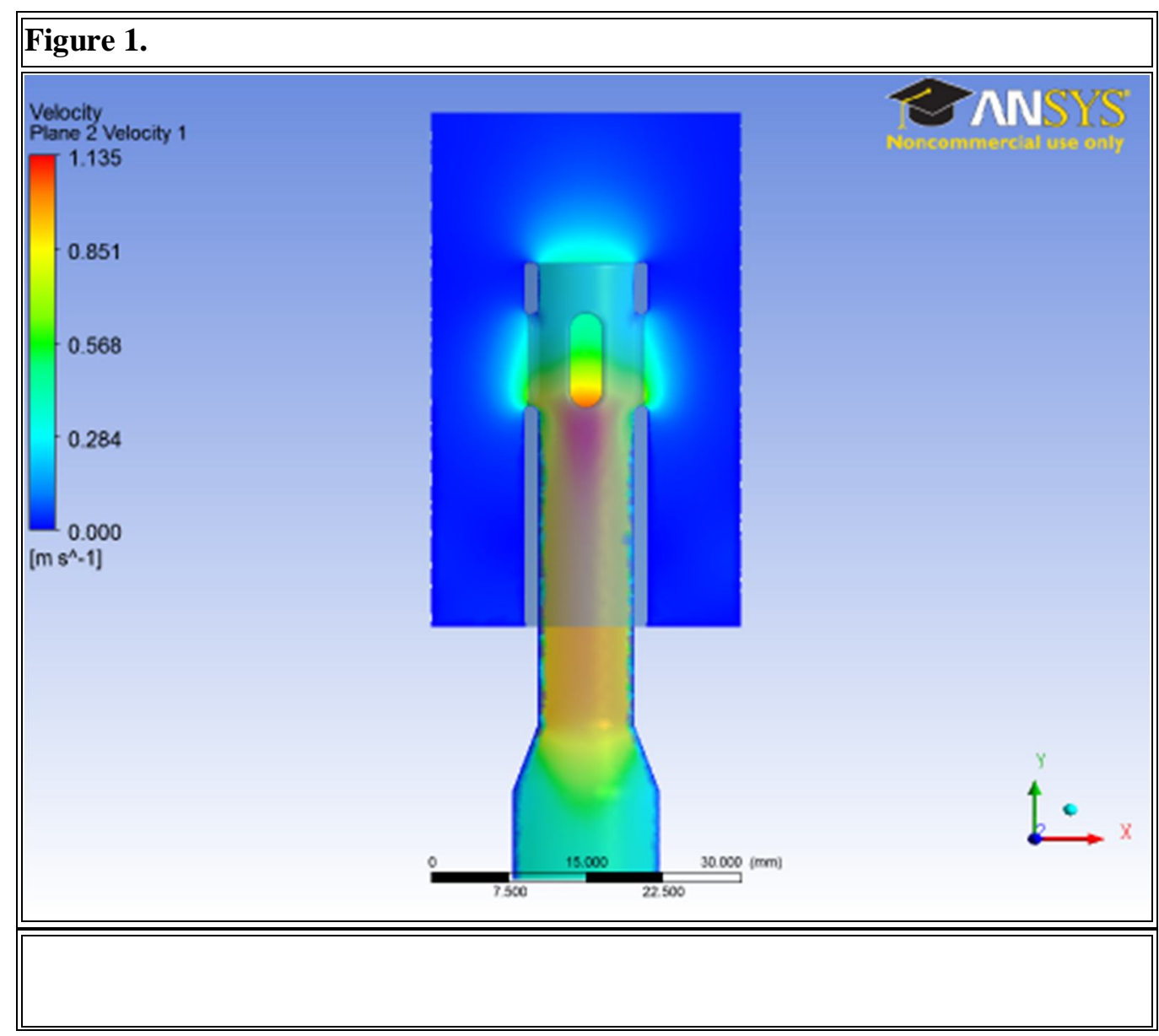


Figure 2.

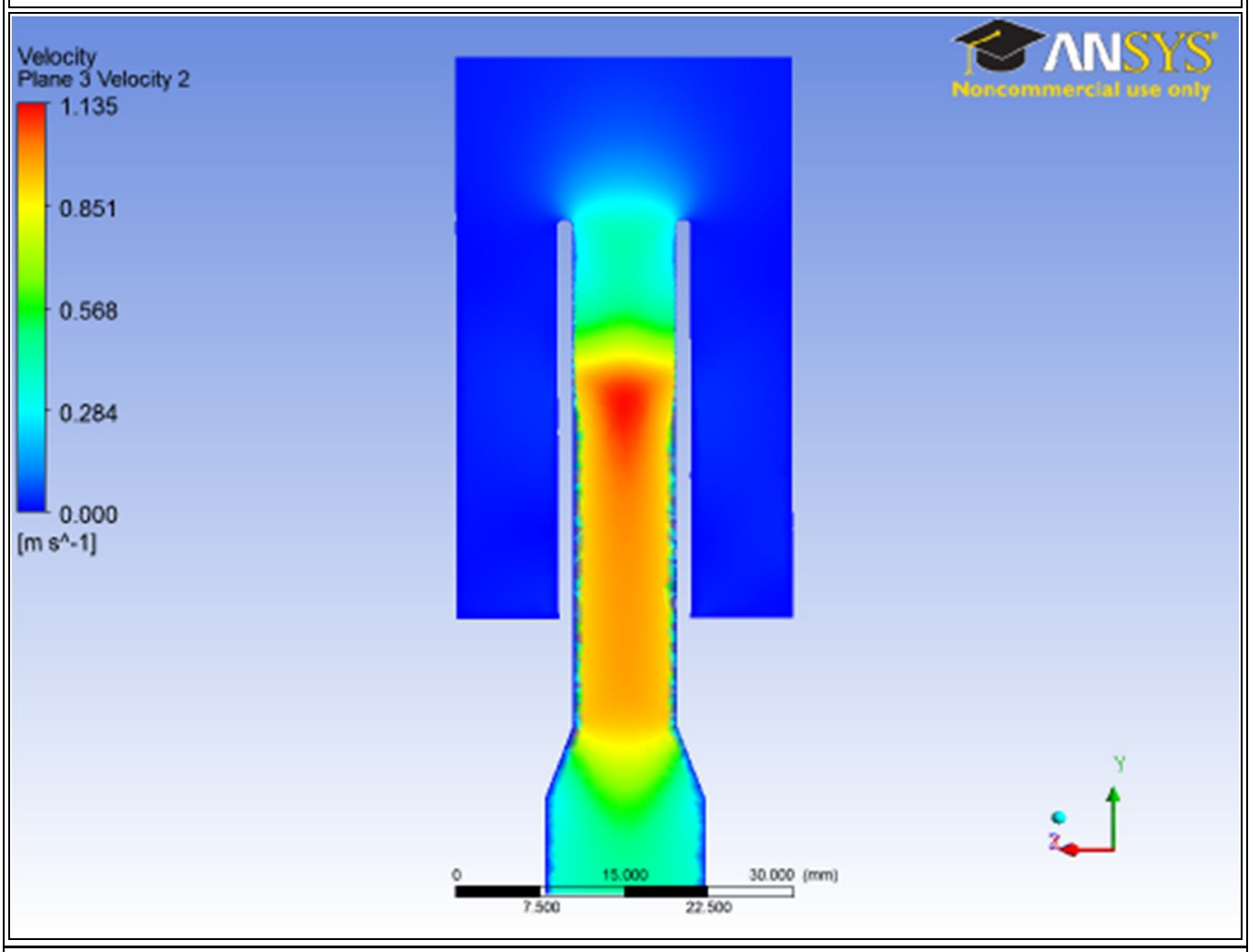

Figure 3.

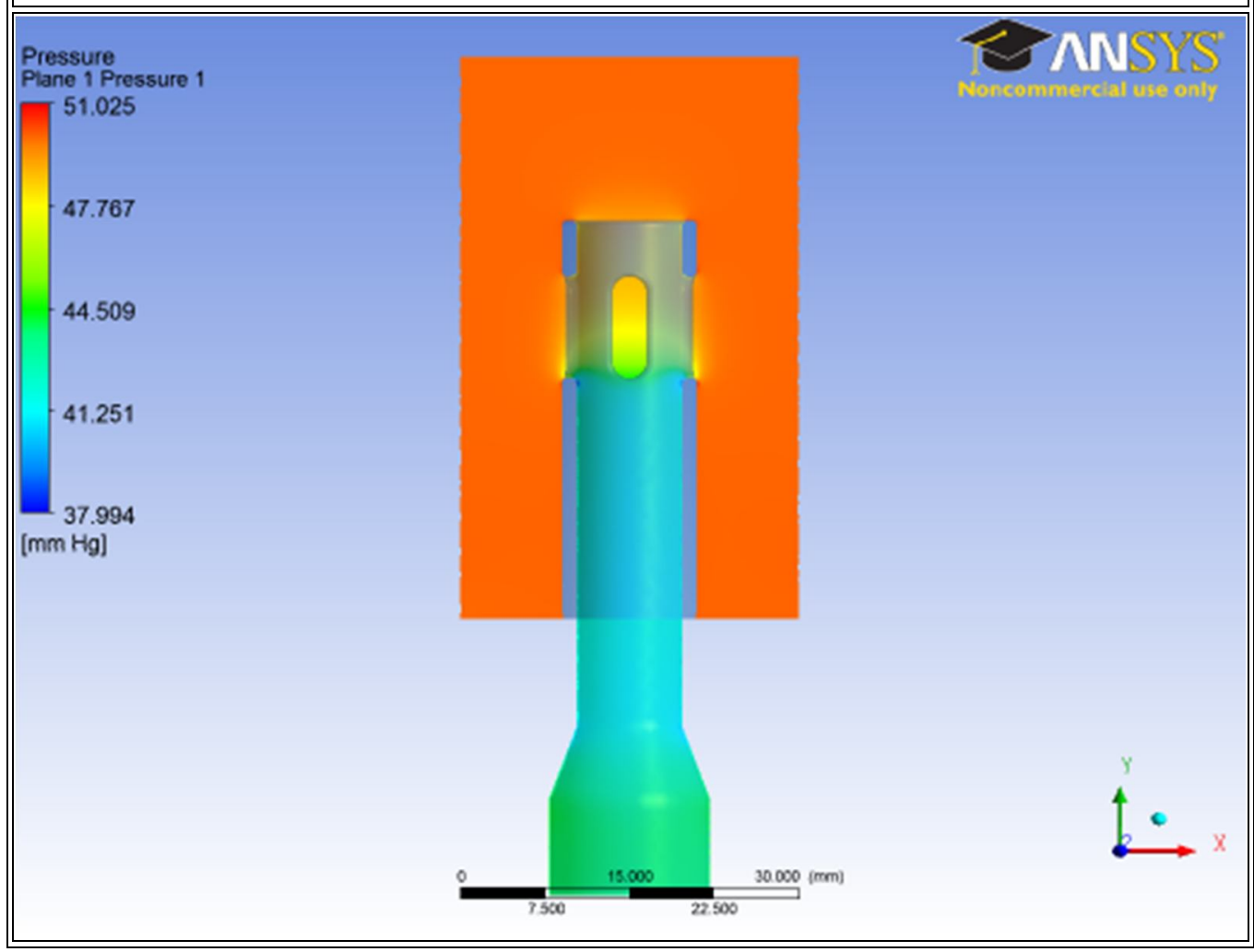


Figure 4.

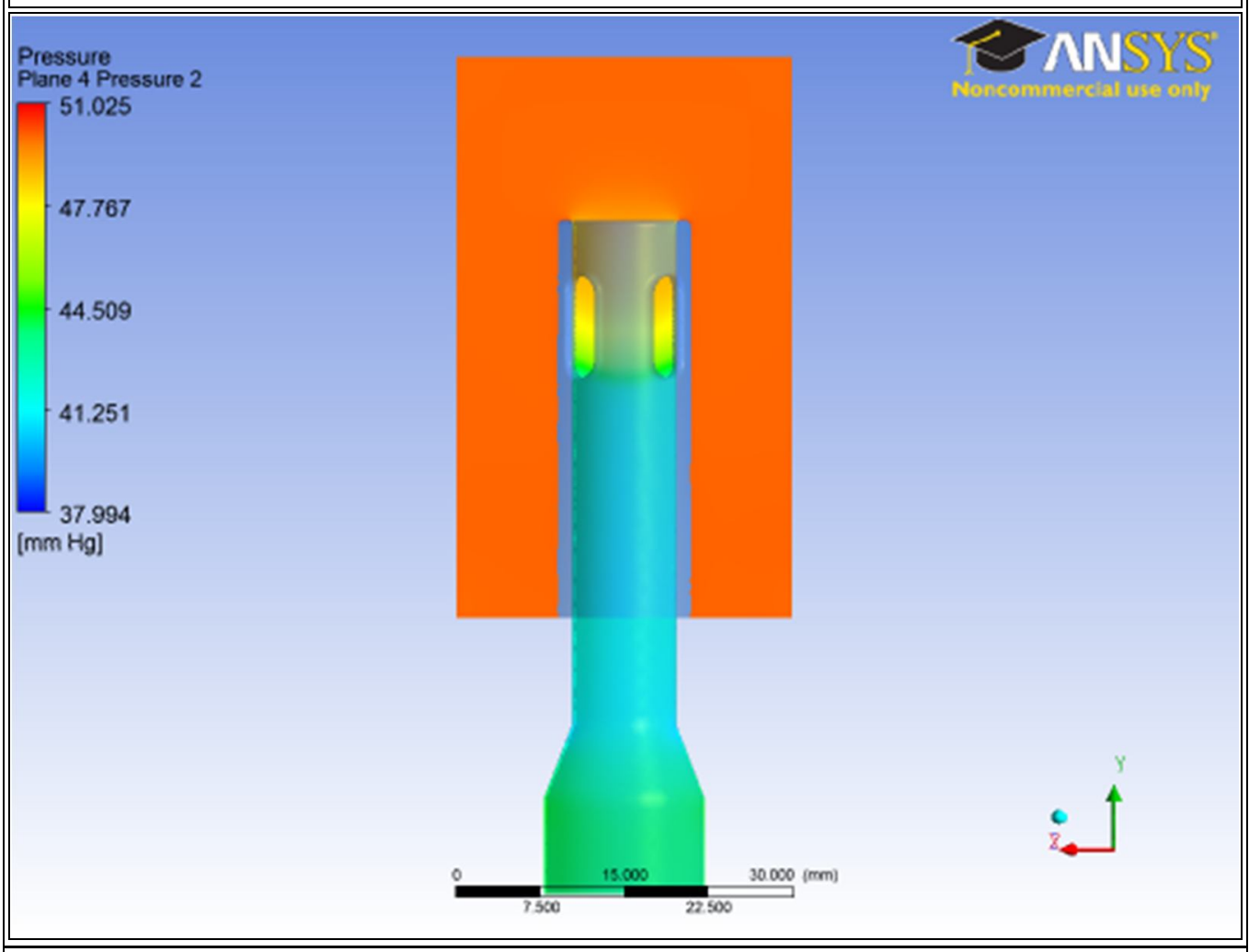

Figure 5. Inside wall shear stress

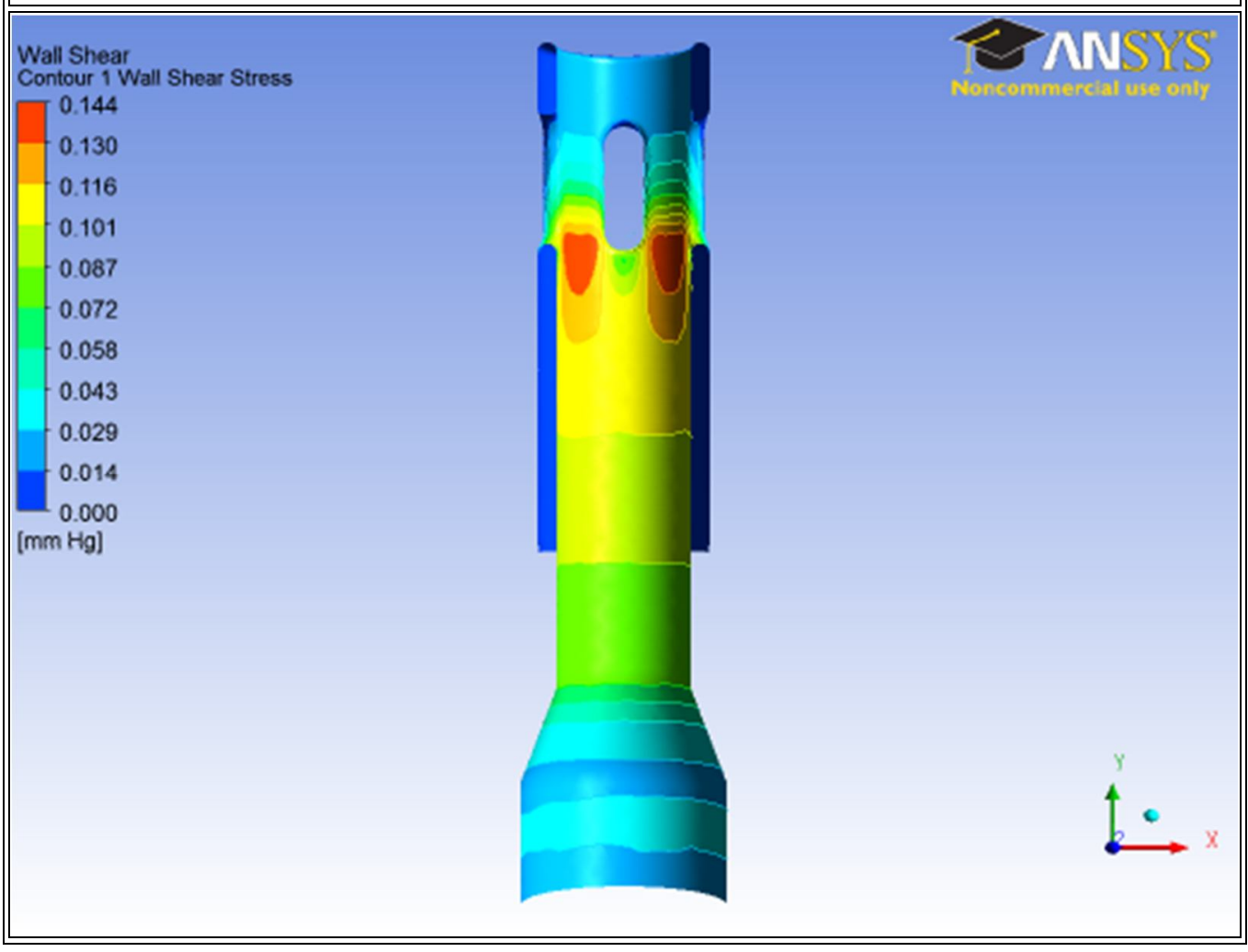



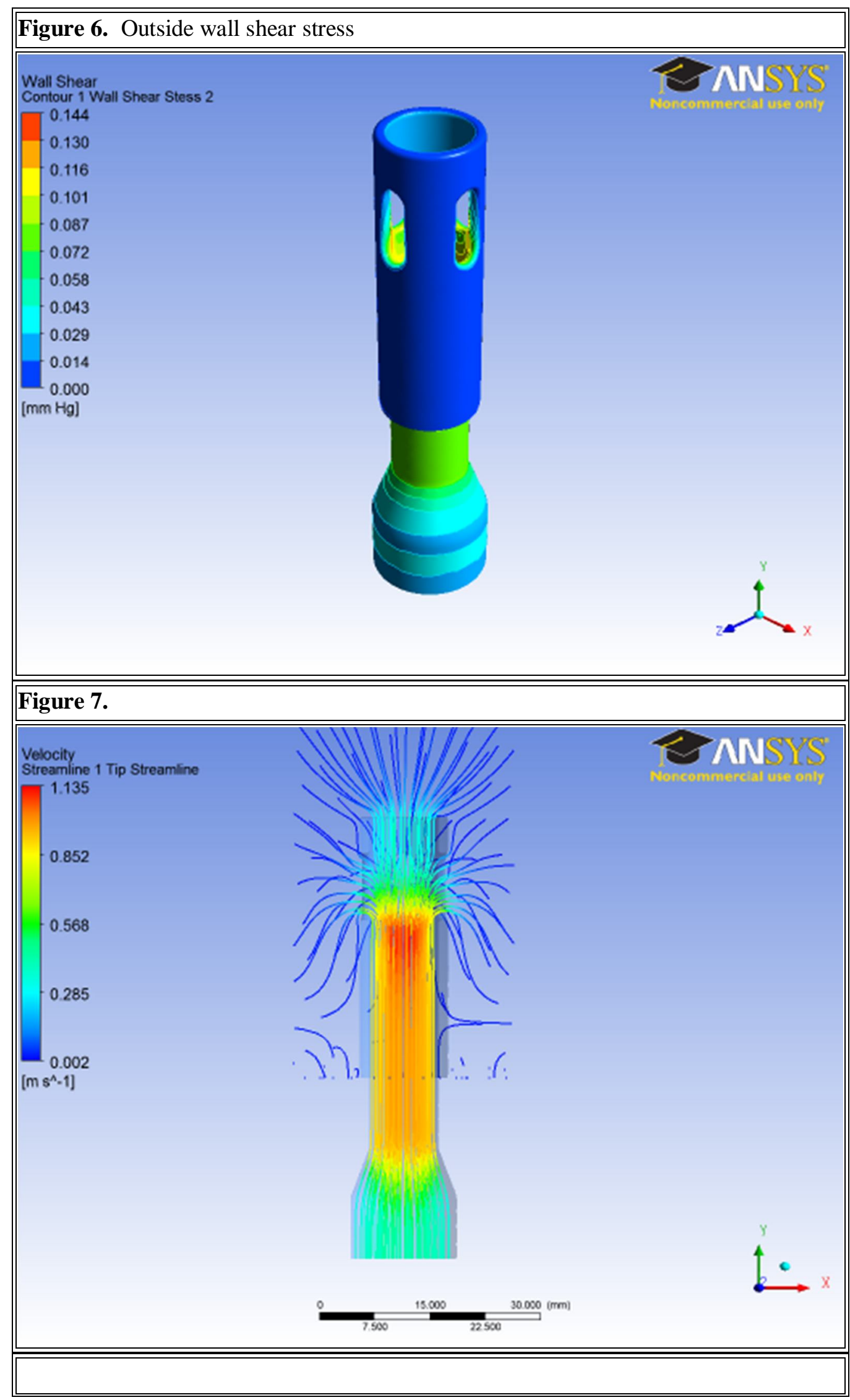

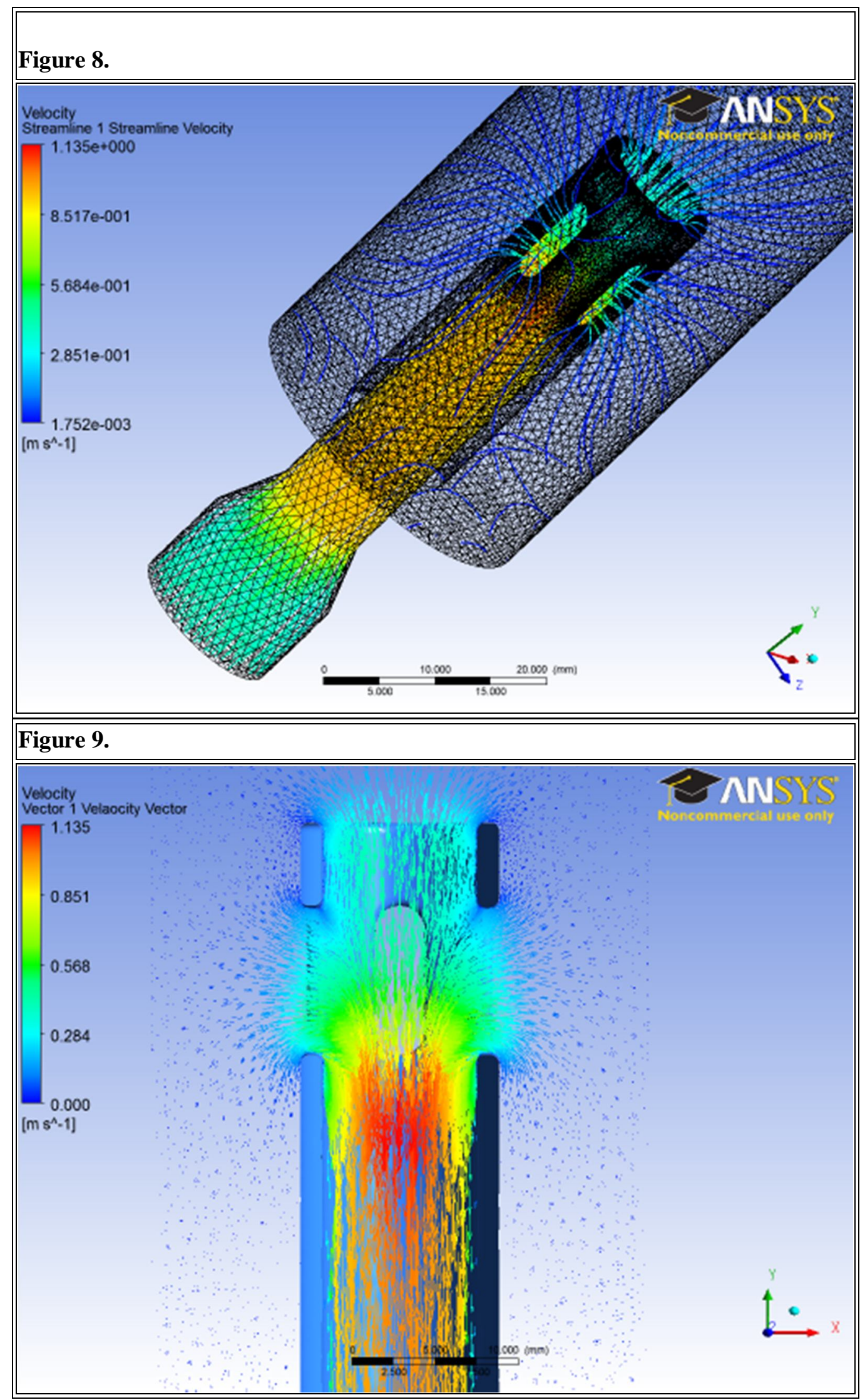


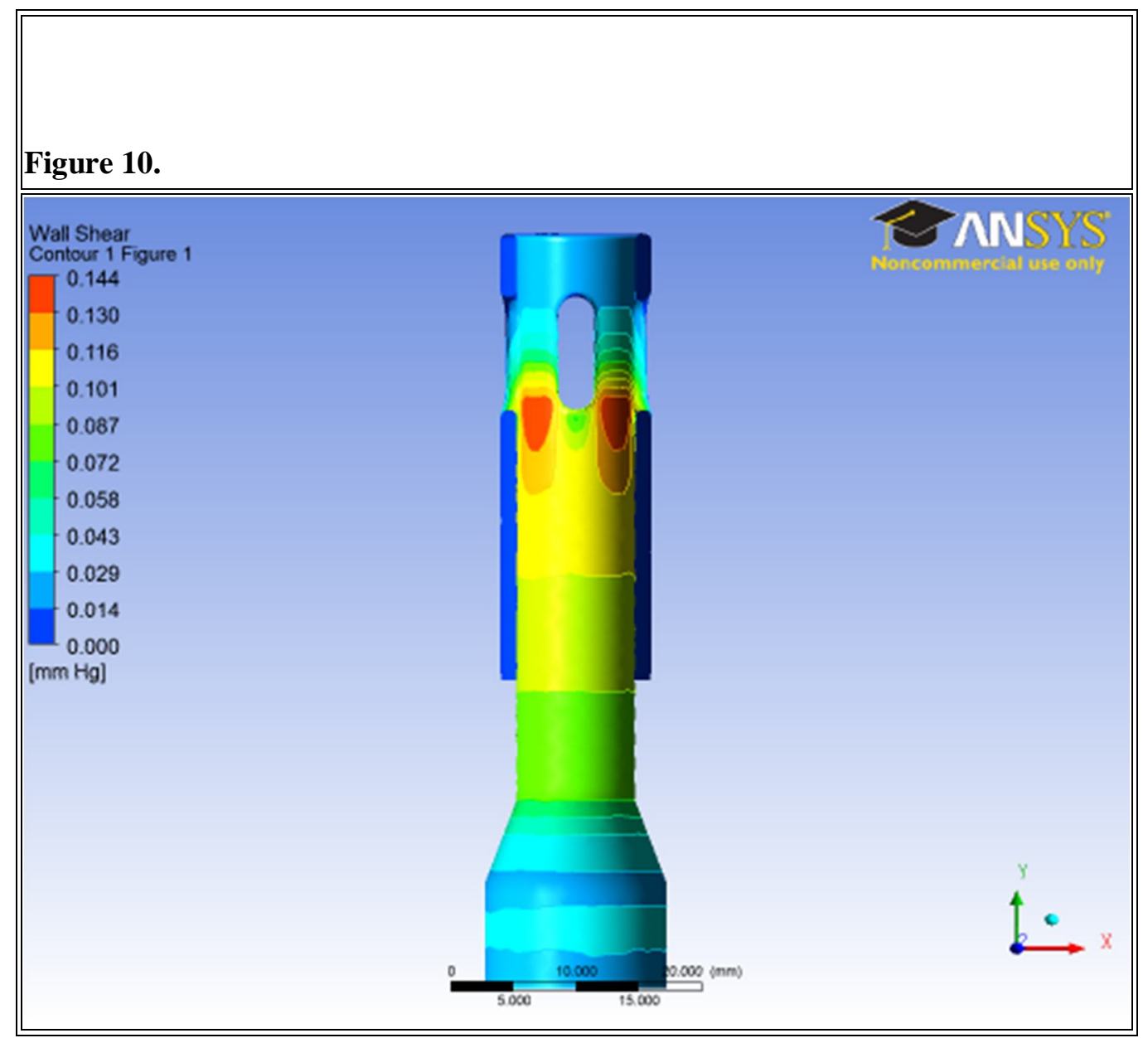

\section{User Data}

\section{Table 7.}

\begin{tabular}{|l||l|l||l||}
\hline Wall Shear Stress & Average & Max & Min \\
\hline Default Domain Default & $2.973 \mathrm{e}-02[\mathrm{~mm} \mathrm{Hg}]$ & $1.444 \mathrm{e}-01[\mathrm{~mm} \mathrm{Hg}]$ & $1.175 \mathrm{e}-05[\mathrm{~mm} \mathrm{Hg}]$ \\
\hline \hline & & & \\
\hline Shear Strain Rate & Average & Max & Min \\
\hline Default Domain Default & $1.876 \mathrm{e}+02\left[\mathrm{~s}^{\wedge}-1\right]$ & $8.514 \mathrm{e}+02\left[\mathrm{~s}^{\wedge}-1\right]$ & $1.594 \mathrm{e}+00\left[\mathrm{~s}^{\wedge}-1\right]$ \\
\hline \hline & & & \\
\hline Volume & & & \\
\hline Default Domain & $3.668 \mathrm{e}-05\left[\mathrm{~m}^{\wedge} 3\right]$ & & \\
\hline \hline & & & \\
\hline \hline Area & & & \\
\hline Inlet 1 & $6.015 \mathrm{e}-03\left[\mathrm{~m}^{\wedge} 2\right]$ & & \\
\hline Outlet & $1.622 \mathrm{e}-04\left[\mathrm{~m}^{\wedge} 2\right]$ & & \\
\hline Default Domain Default & $3.212 \mathrm{e}-03\left[\mathrm{~m}^{\wedge} 2\right]$ & & \\
\hline
\end{tabular}




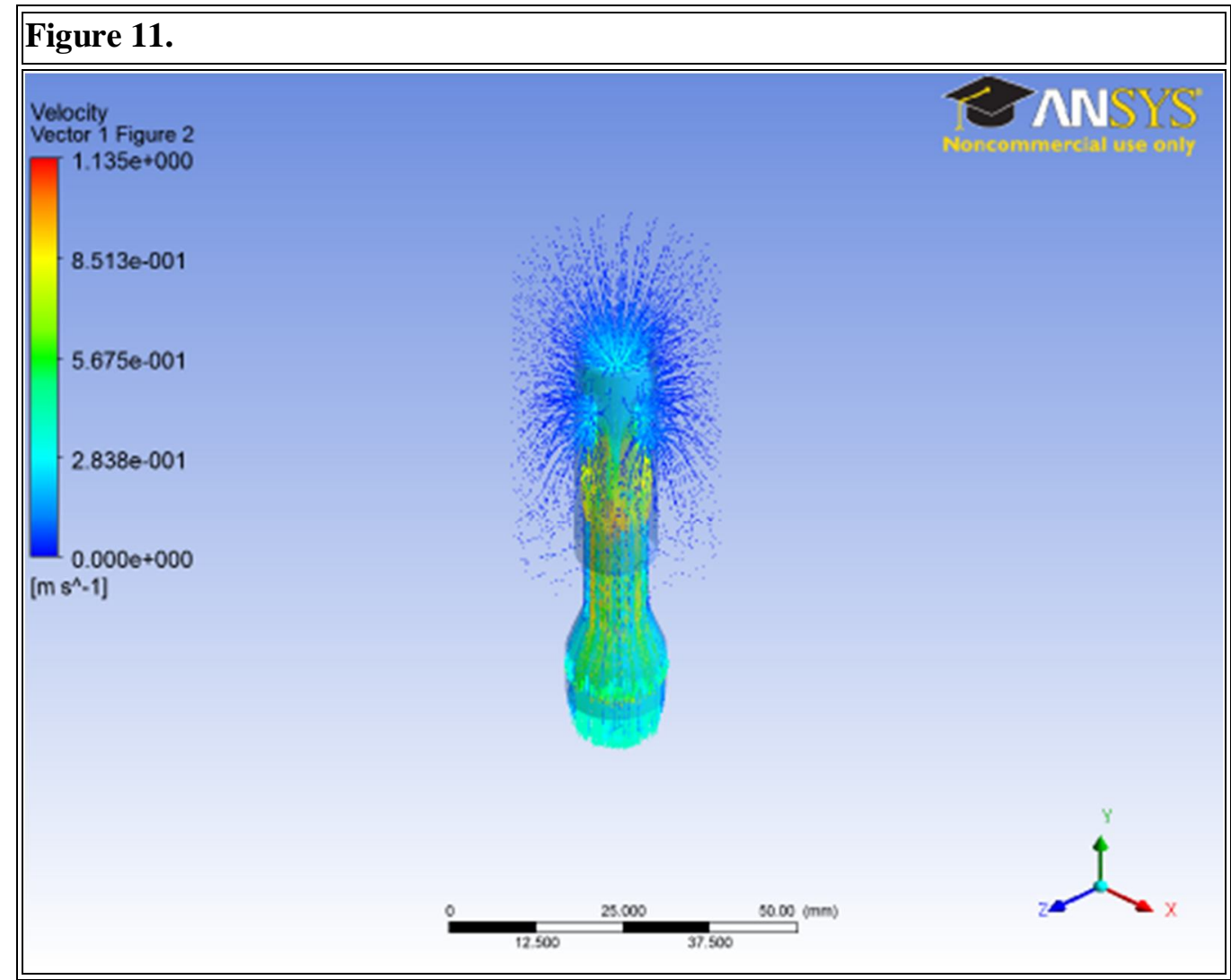

